\title{
Intramolecular Cyclopropanation of Unsaturated Terminal Epoxides and Chlorohydrins
}

\author{
David M. Hodgson, ${ }^{*}{ }^{\dagger}$ Ying Kit Chung, ${ }^{\dagger}$ Irene Nuzzo, ${ }^{\dagger}$ Glòria Freixas, ${ }^{\dagger}$ Krystyna K. \\ Kulikiewicz, ${ }^{\dagger}$ Ed Cleator ${ }^{\ddagger}$ and Jean-Marc Paris ${ }^{\S}$ \\ ${ }^{\dagger}$ Department of Chemistry, University of Oxford, Chemistry Research Laboratory, Mansfield Road, Oxford OXI 3TA, \\ U.K. \\ ${ }^{7}$ Department of Process Research, Merck Sharp and Dohme Limited, Hertford Road, Hoddesdon, Hertfordshire, EN11 \\ $9 B U, U . K$.

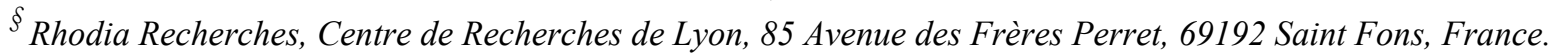

\section{Full list of authors for references from main paper with 16 or more authors}

[3a] Bueno, A. B.; Collado, I.; de Dios, A.; Dominguez, C.; Martin, J. A.; Martin, L. M.; MartinezGrau, M. A.; Montero, C.; Pedregal, C.; Catlow, J.; Coffey, D. S.; Clay, M. P.; Dantzig, A. H.; Lindstrom, T.; Monn, J. A.; Jiang, H.; Schoepp, D. D.; Stratford, R. E.; Tabas, L. B.; Tizzano, J. P.; Wright, R. A.; Herin, M. F. J. Med. Chem. 2005, 48, 5305-5320.

[3b] McBriar, M. D.; Guzik, H.; Shapiro, S.; Paruchova, J.; Xu, R.; Palani, A.; Clader, J. W.; Cox, K.; Greenlee, W. J.; Hawes, B. E.; Kowalski, T. J.; O’Neill, K.; Spar, B. D.; Weig, B.; Weston, D. J.; Farley, C.; Cook, J.; J. Med. Chem. 2006, 49, 2294-2310.

\section{General details}

All reactions requiring anhydrous conditions were conducted in flame-dried apparatus under an atmosphere of argon or nitrogen. THF and $\mathrm{Et}_{2} \mathrm{O}$ were distilled from benzophenone ketyl; $t$-BuOMe, 2,2,6,6-tetramethylpiperidine (TMP) and 1,2-epoxy-5-hexene from $\mathrm{CaH}_{2}$; all other reagents were used as received, unless stated otherwise. Column chromatography was carried out on silica gel (Kieselgel 60, 40-63 $\mu \mathrm{m}$ ) as stationary phase. TLC was performed on aluminium sheets pre-coated with silica (Merck Silica Gel $60 \mathrm{~F}_{254}$ ), which were visualised by staining either with 5\% w/v phosphomolybdic acid in $\mathrm{EtOH}$, or $\mathrm{KMnO}_{4}$ solutions. Petrol refers to the petroleum fraction boiling between $30{ }^{\circ} \mathrm{C}$ and $40{ }^{\circ} \mathrm{C}$. IR spectra were recorded at $\mathrm{rt}$ on a Perkin-Elmer 1750 FTIR 
spectrophotometer using $\mathrm{KBr}$ discs or thin films on film plates as stated. Peak intensities are specified as broad (b), strong (s), medium (m) or weak (w), and all absorptions are reported in wavenumbers $\left(\mathrm{cm}^{-1}\right)$. Proton $\left({ }^{1} \mathrm{H}\right)$ and carbon $\left({ }^{13} \mathrm{C}\right)$ NMR spectra were recorded in $\mathrm{CDCl}_{3}$ at room temperature, with Bruker DPX250, DPX400, DQX400 or AMX500 spectrometers unless stated otherwise; Chemical shifts $\left(\delta_{\mathrm{H}}\right.$ and $\left.\delta_{\mathrm{C}}\right)$ are reported in parts per million $(\mathrm{ppm})$, and are referenced relative to the $\mathrm{CHCl}_{3}\left({ }^{1} \mathrm{H} \mathrm{NMR}\right.$ 7.27) and $\mathrm{CDCl}_{3}\left({ }^{13} \mathrm{C} \mathrm{NMR} 77.0\right.$, central signal of triplet) signals, unless stated otherwise. Assignments were aided by COSY, DEPT, HMQC and NOE experiments. Coupling constants $(J)$ are given in Hertz to the nearest $\pm 0.1 \mathrm{~Hz}$. Multiplicities are given as singlet (s), doublet (d), triplet (t), quartet (q), quintet (quin), multiplet (m) and broad (b) where applicable. Diastereotopic protons in the molecule are assigned as $\mathrm{H}-\mathrm{X}$ and $\mathrm{H}-\mathrm{X}^{\prime}$ in the NMR spectra, where $\mathrm{X}$ is the proton number indicated in the structure. Mass spectra $(\mathrm{m} / \mathrm{z}$, low and high resolution) were recorded on a Micromass GCT spectrometer using the techniques of chemical ionisation (CI+) or field ionisation (FI+), or by the EPSRC National Mass Spectrometry Service Centre at the University of Swansea on a 900 XLT high resolution double focusing mass spectrometer using the technique of electrospray ionisation (ES+). Chiral stationary phase HPLC was performed using a Daicel Chiralcel OD column $(4.6 \mathrm{~mm} \times 250 \mathrm{~mm})$ or Daicel Chiralpak AD column $(4.6 \mathrm{~mm} \times 250$ $\mathrm{mm}$ ) on a Gilson System with 712 Controller Software and a $118 \mathrm{UV} / \mathrm{vis}$ detector at the appropriate wavelength for the sample under analysis. Chiral GC analysis was performed using a ThermoQuest CE Instruments TRACE GC, running Chrom-Card for TRACE software, fitted with a CYDEX- $\beta$ column at the stated temperature. All enantioenriched samples were verified by the preparation of racemic samples. Enantiomeric excesses (ees) were determined by chiral HPLC or GC to the nearest $1 \%$. Retention times $\left(t_{\mathrm{R}}\right)$ are given in minutes to the nearest $0.01 \mathrm{~min}$. Optical rotations $[\alpha]^{\mathrm{T}}{ }_{\mathrm{D}}$ were measured using a Perkin Elmer 241 Polarimeter with a cell of path length $1.0 \mathrm{dm}$, at $\mathrm{T}^{\circ} \mathrm{C}$ and are given in $10^{-1} \mathrm{deg} \mathrm{cm}^{2} \mathrm{~g}^{-1}$. Concentrations $(c)$ are given in $\mathrm{g} / 100 \mathrm{~mL}$. 


\section{Optimization of the intramolecular cyclopropanation reaction using 1,2- epoxy-5-hexene $4 a$}

For our initial study, direct application of Yamamoto's conditions to epoxide $\mathbf{4 a}$ [addition of epoxide ( $1 \mathrm{mmol}$ ) to a solution of LTMP (2 equiv., $0.2 \mathrm{M}$ in THF, $\left.\left.25{ }^{\circ} \mathrm{C}, 1 \mathrm{~h}\right)\right]$ gave alcohol $\mathbf{5 a}$ in $47 \%$ yield (Table 1, entry 1). Volatile 5-hexanal (derived from hydrolysis of the corresponding enamine) was also observed on TLC, indicating that enamine formation was in competition with the cyclopropanation process. Since enamination proceeds efficiently in THF but is sluggish in $\mathrm{Et}_{2} \mathrm{O},{ }^{[1]}$ and is conducted at $\mathrm{rt}$, therefore we envisaged that the use of $\mathrm{Et}_{2} \mathrm{O}$ as solvent and a lower addition temperature $\left(0{ }^{\circ} \mathrm{C}\right)$ in subsequent runs might favor the intramolecular cyclopropanation pathway. The rate of LTMP addition to the epoxide was investigated with these changes implemented. Solutions of LTMP at $0{ }^{\circ} \mathrm{C}$ were added via cannula to stirred solutions of $4 \mathbf{a}$ at $0{ }^{\circ} \mathrm{C}$ over 15,40 and $60 \mathrm{~min}$, resulting in the isolation of 5a in 67, 66 and 79\% yields, respectively (entries 2 - 4). There was no significant difference in the yield within experimental error as the rate varied between 15 to $60 \mathrm{~min}$; thus an addition time of 40 to $60 \mathrm{~min}$ was considered to be the most practical for controlled addition of a relatively small amount of LTMP solution via cannula. Subsequently, epoxide 4a was treated with LTMP and the resulting mixture was only allowed to react at room temperature for $2 \mathrm{~h}$ (entry 5). This led to a 1:1 mixture of $\mathbf{4 a}$ and $\mathbf{5 a}$ in the crude product after ${ }^{1} \mathrm{H}$ NMR analysis, and we concluded that the cyclopropanation reaction is significantly slower than enamine formation, which requires only $1 \mathrm{~h}$. We then investigated the molar equivalents of the base required for the cyclopropanation reaction by changing the amount of LTMP to 1.5 and 2.5 equiv. Incomplete consumption of $\mathbf{4 a}$ was observed with 1.5 equiv. of LTMP (entry 6), and 5a was isolated in only $62 \%$ yield when 2.5 equiv. of LTMP was used (entry 7). This indicates the use of 2 equiv. of LTMP is optimal. The reaction was reexamined in hexane and THF under the conditions applied in entry 4 (entries 8 and 9). Bicyclic alcohol 5a was cleanly isolated in 62\% yield from the hexane reaction. In contrast, ${ }^{1} \mathrm{H}$ NMR analysis of $\mathbf{5 a}$ isolated after column chromatography from the THF reaction revealed a significant amount of side-products $(\sim 10 \%)$ present, which gave rise to distinctive vinylic signals $(\delta 4.7-5.7 \mathrm{ppm})$. The latter side-products were postulated to be a mixture of cis- and transcyclopropanes $9(\mathrm{R}=\mathrm{H}$, ratio not determined), formed via allylic deprotonation followed by 
intramolecular epoxide ring-opening. Apparu and Barelle ${ }^{[2,3]}$ have previously reported the formation of trans-9 in the reaction between $4 \mathbf{a}$ and $N$-lithioethylenediamine in HMPA as solvent at room temperature. However, their $\mathrm{NMR}$ data were obtained in $\mathrm{CCl}_{4}$, making direct comparison difficult.

Table 1. Optimization of the intramolecular cyclopropanation reaction

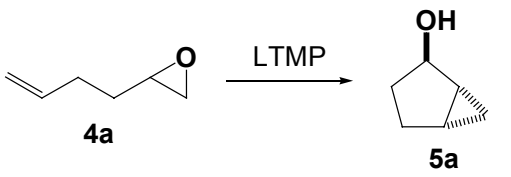

\begin{tabular}{ccccc}
\hline Entry $^{a}$ & $\begin{array}{c}\text { LTMP }^{b} \\
\text { (equiv.) }\end{array}$ & Solvent & Time (h) & ${\text { Yield }(\%)^{c}}^{c}$ \\
\hline 1 & 2 & $\mathrm{THF}$ & 1 & 47 \\
$2^{d}$ & 2 & $\mathrm{Et}_{2} \mathrm{O}$ & 40 & 67 \\
$3^{e}$ & 2 & $\mathrm{Et}_{2} \mathrm{O}$ & 40 & 66 \\
$4^{f}$ & 2 & $\mathrm{Et}_{2} \mathrm{O}$ & 12 & 79 \\
5 & 2 & $\mathrm{Et}_{2} \mathrm{O}$ & 2 & $\mathbf{4 a : 5 a}=1: 1^{g}$ \\
6 & 1.5 & $\mathrm{Et}_{2} \mathrm{O}$ & 20 & $\mathbf{4 a}=29, \mathbf{5 a}=46$ \\
7 & 2.5 & $\mathrm{Et}_{2} \mathrm{O}$ & 18 & 62 \\
8 & 2 & $\mathrm{Hexane}$ & 16 & 62 \\
9 & 2 & $\mathrm{THF}$ & 16 & $48^{h}$
\end{tabular}

${ }^{a}$ Performed with $1 \mathrm{mmol}$ of $\mathbf{4 a}$ at $0.2 \mathrm{M} .{ }^{b} 0.2 \mathrm{M} .{ }^{c}$ Isolated yields. ${ }^{d}$ LTMP added over $15 \mathrm{~min} .{ }^{e}$ LTMP added over 40 min. ${ }^{f}$ LTMP added over 60 min. ${ }^{g}$ Crude residue analyzed by ${ }^{1} \mathrm{H}$ NMR. ${ }^{h}$ The presence of $9(\mathrm{R}=\mathrm{H}, \sim 10 \%)$ observed in ${ }^{1} \mathrm{H}$ NMR.

\section{Typical cyclopropanation procedure: formation of bicyclic alcohol 5a from terminal epoxide 4a and LTMP}

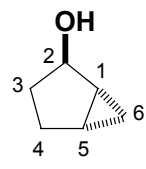

$n$-BuLi (1.6 M in hexane, $1.3 \mathrm{~mL}, 2.0 \mathrm{mmol})$ was added to a stirred solution of 2,2,6,6tetramethylpiperidine $(0.34 \mathrm{~mL}, 2.00 \mathrm{mmol})$ in $t$-BuOMe $(10 \mathrm{~mL})$ at $-78{ }^{\circ} \mathrm{C}$. The pale yellow LTMP solution formed was stirred at $\mathrm{rt}$ for $15 \mathrm{~min}$ and cooled to $0{ }^{\circ} \mathrm{C}$ in an ice bath. To a stirred solution of unsaturated terminal epoxide $(1.00 \mathrm{mmol})$ in $t$-BuOMe $(5 \mathrm{~mL})$ at $0{ }^{\circ} \mathrm{C}$ was added the LTMP solution dropwise via cannula over 45 - $60 \mathrm{~min}$. The resulting mixture was stirred at $\mathrm{rt}$ for the duration specified, quenched with $\mathrm{MeOH}(0.5 \mathrm{~mL})$ and concentrated. The residue was dryloaded onto a small amount of silica and purified by column chromatography $\left(30 \% \mathrm{Et}_{2} \mathrm{O}\right.$ in petrol) to give bicyclo[3.1.0]hexan-2-ol $\mathbf{5 a}^{[3]}(77 \mathrm{mg}, 0.79 \mathrm{mmol}, 79 \%)$ as a pale yellow oil. 


\section{Procedures for the formation of bicyclic alcohols 5a-q from epoxides $4 a-$ $q$ and LTMP (Table 1 in main paper)}

\section{(+)-Bicyclo[3.1.0]hexan-2-ol (+)-5a}

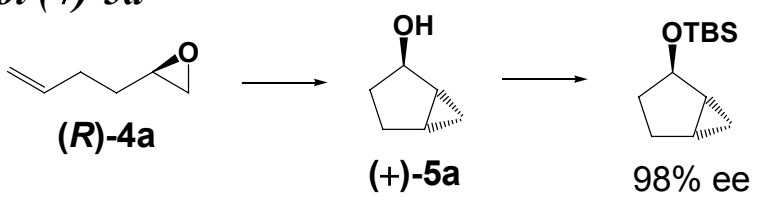

(R)-1,2-Epoxy-5-hexene $(\boldsymbol{R})-\mathbf{4 a}$

According to Jacobsen's procedure ${ }^{[4]} \quad(R, R)-N, N^{\prime}$-Bis(3,5-di-tert-butylsalicylidinene)-1,2cyclohexanediaminocobalt (II) (495 mg, $0.82 \mathrm{mmol}, 2 \mathrm{~mol} \%$ ) was treated with 1,2-epoxy-5-hexene 4a (4.00 g, $40.8 \mathrm{mmol})$, AcOH (47 $\mu 1,0.82 \mathrm{mmol}, 0.02$ equiv.) and THF ( $0.5 \mathrm{~mL})$. The mixture was cooled to $0{ }^{\circ} \mathrm{C}$, followed by addition of water (403 $\mu 1,22.4 \mathrm{mmol}, 0.55$ equiv.) in one portion and stirred at rt for $20 \mathrm{~h}$. Subjection of the crude mixture to bulb-to-bulb Kugelrohr distillation (at rt and 0.07 mbar) gave $(R)$-1,2-epoxy-5-hexene $(\boldsymbol{R})-4 a(1.54 \mathrm{~g}, 15.7 \mathrm{mmol}, 39 \%)$ as a colorless liquid; $[\alpha]_{\mathrm{D}}^{22}=+5.3\left(c, 1.0\right.$ in $\left.\mathrm{CHCl}_{3}\right)\left\{\right.$ lit. $^{[4]}=[\alpha]_{\mathrm{D}}{ }^{25}=+9.36$ (neat) $\} ;{ }^{1} \mathrm{H}$ and ${ }^{13} \mathrm{C}$ NMR spectra were identical to the starting epoxide. The enantiomeric excess of $(\boldsymbol{R})-\mathbf{4 a}(\mathrm{ee}>98 \%)$ was confirmed by chiral HPLC (OD column, 0.1/0.4 $\mathrm{mLmin}^{-1} / 98.5 \%$ heptane and 1.5\% EtOH) analysis of the 2naphthalene thiol derivative. ${ }^{[5]}$

\section{(+)-Bicyclo[3.1.0]hexan-2-ol (+)-5a}

Following the typical cyclopropanation procedure, $(R)$-1,2-epoxy-5-hexene $(\boldsymbol{R})$-4a $(196 \mathrm{mg}, 2.0$ mmol) was treated with LTMP in $\mathrm{Et}_{2} \mathrm{O}$ for $16 \mathrm{~h}$ to give (+)-bicyclo[3.1.0]hexan-2-ol (+)-5a (137 mg, $1.40 \mathrm{mmol}, 70 \%)$ as a pale yellow oil, $[\alpha]_{\mathrm{D}}^{22}=+31.0\left(c, 1.0\right.$ in $\left.\mathrm{CHCl}_{3}\right)$. NMR data see 5a above. The enantiomeric excess of $(+)-5 a(e e=98 \%)$ was confirmed by chiral GC (Cydex- $\beta$ column, 80 ${ }^{\circ} \mathrm{C} / 0.6 \mathrm{mLmin}^{-1}$ ) analysis of the tert-butyldimethylsilyl derivative below:

tert-Butyldimethylsilyl chloride (230 mg, $1.53 \mathrm{mmol}, 1.5$ equiv.) was added to a stirred solution of (+)-bicyclo[3.1.0]hexan-2-ol (+)-5a (100 mg, $1.02 \mathrm{mmol})$ and imidazole (139 mg, 2.04 mmol, 2.0 equiv.) in $\mathrm{CH}_{2} \mathrm{Cl}_{2}(4 \mathrm{~mL})$ at $\mathrm{rt}$. The reaction mixture was stirred for $4 \mathrm{~h}$, then diluted with 
$\mathrm{CH}_{2} \mathrm{Cl}_{2}(50 \mathrm{~mL})$, washed with sat. aq. $\mathrm{NaHCO}_{3}(50 \mathrm{~mL})$ and the layers were separated. The organic layer was dried $\left(\mathrm{MgSO}_{4}\right)$, filtered and concentrated to give the crude product. Purification by column chromatography $\left(10 \% \mathrm{Et}_{2} \mathrm{O}\right.$ in petrol) gave the desired chiral silylether $(178 \mathrm{mg}, 0.84$ mmol, $82 \%)$ as a colorless oil; $R_{\mathrm{f}}=0.7\left(10 \% \mathrm{Et}_{2} \mathrm{O}\right.$ in petrol $) ;[\alpha]_{\mathrm{D}}^{22}=+8.7\left(c, 1.0\right.$ in $\left.\mathrm{CHCl}_{3}\right) ; e e=$ 98\%; IR (film) 2950s (C-H), 1466m, 1365m, 1253s (C-Si), 1106s (C-O), 1060s, 1023s; ${ }^{1} \mathrm{H}$ NMR $(400 \mathrm{MHz})-0.07--0.04(\mathrm{~m}, 1 \mathrm{H}, \mathrm{H}-6), 0.08$ (s, 3H, $\left.\mathrm{SiCH}_{3}\right), 0.09$ (s, 3H, SiCH 3$), 0.33-0.38$ (m, 1H, H-6'), 0.91 (s, 9H, $\left.\mathrm{SiC}\left(\mathrm{CH}_{3}\right)_{3}\right), 1.24-1.34$ (m, 2H, H-1, H-4'), 1.38 - 1.43 (m, 1H, H-5), 1.46 (dd, 1H, H-4, $J=8.8,14.0), 1.60(\mathrm{dd}, 1 \mathrm{H}, \mathrm{H}-3, J=8.8,12.4), 1.90-2.01\left(\mathrm{~m}, 1 \mathrm{H}, \mathrm{H}-3{ }^{\prime}\right), 4.22(\mathrm{~d}, 1 \mathrm{H}$, $\left.\mathrm{H}-2, J=4.8) ;{ }^{13} \mathrm{C} \mathrm{NMR}\left(\mathrm{CDCl}_{3}, 100 \mathrm{MHz}\right)-4.5\left(\mathrm{Si}\left(\mathrm{CH}_{3}\right)_{2}\right), 6.5(\mathrm{C}-6), 16.7(\mathrm{C}-5), 18.3\left(\mathrm{SiC}_{(\mathrm{CH}}\right)_{3}\right)$, 24.5 (C-1), $24.9(\mathrm{C}-3), 26.0\left(\mathrm{SiC}\left(\mathrm{CH}_{3}\right)_{3}\right), 31.0(\mathrm{C}-4), 75.0(\mathrm{C}-2)$; $\mathrm{MS}(\mathrm{CI}+) \mathrm{m} / z: 213.2\left(\mathrm{MH}^{+}, 100 \%\right)$, $172.1(15 \%), 155.1(21 \%), 133.1(4 \%)$; HRMS $m / z: \mathrm{MH}^{+}$found 213.1670, $\mathrm{C}_{12} \mathrm{H}_{25} \mathrm{OSi}$ requires 213.1675 .
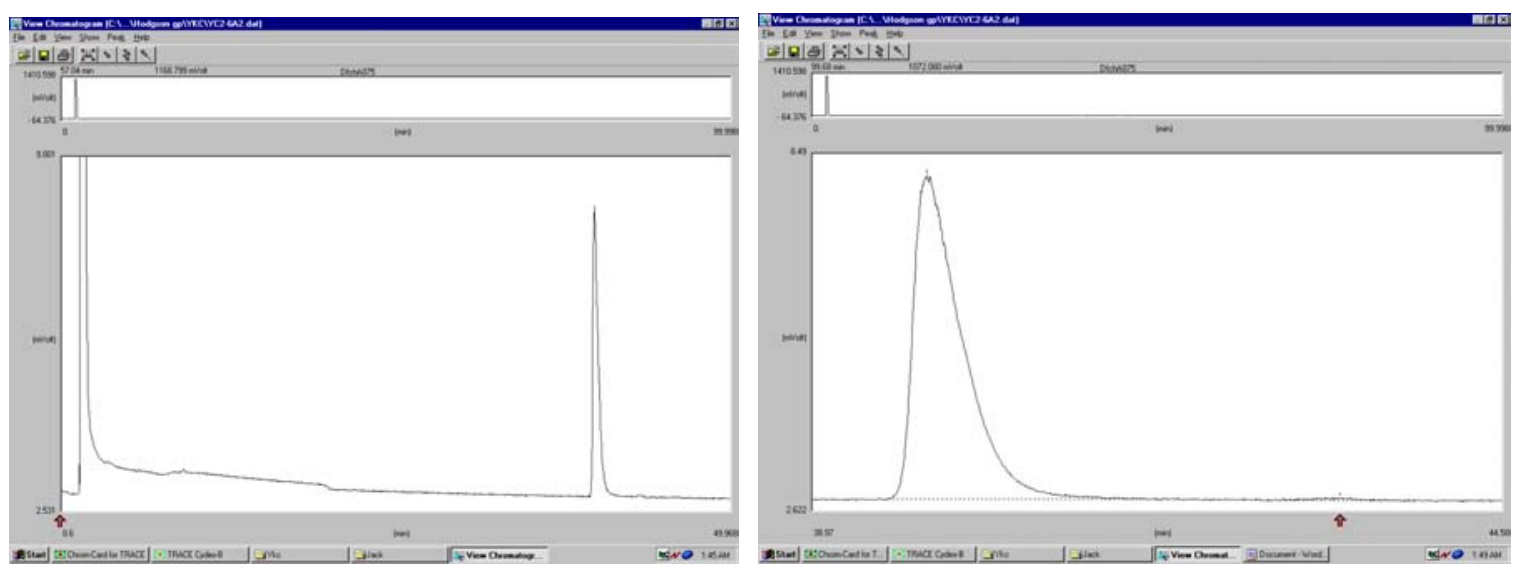

\begin{tabular}{crrr} 
Peak Number & Area \% & Ret.Time & Area BC \\
\hdashline 1 & 99.2157 & 39.90 & $1449302 \mathrm{mi}$ \\
2 & 0.7843 & 43.26 & $11457 \mathrm{mi}$ \\
Totals & 100.0000 & & 1460759
\end{tabular}



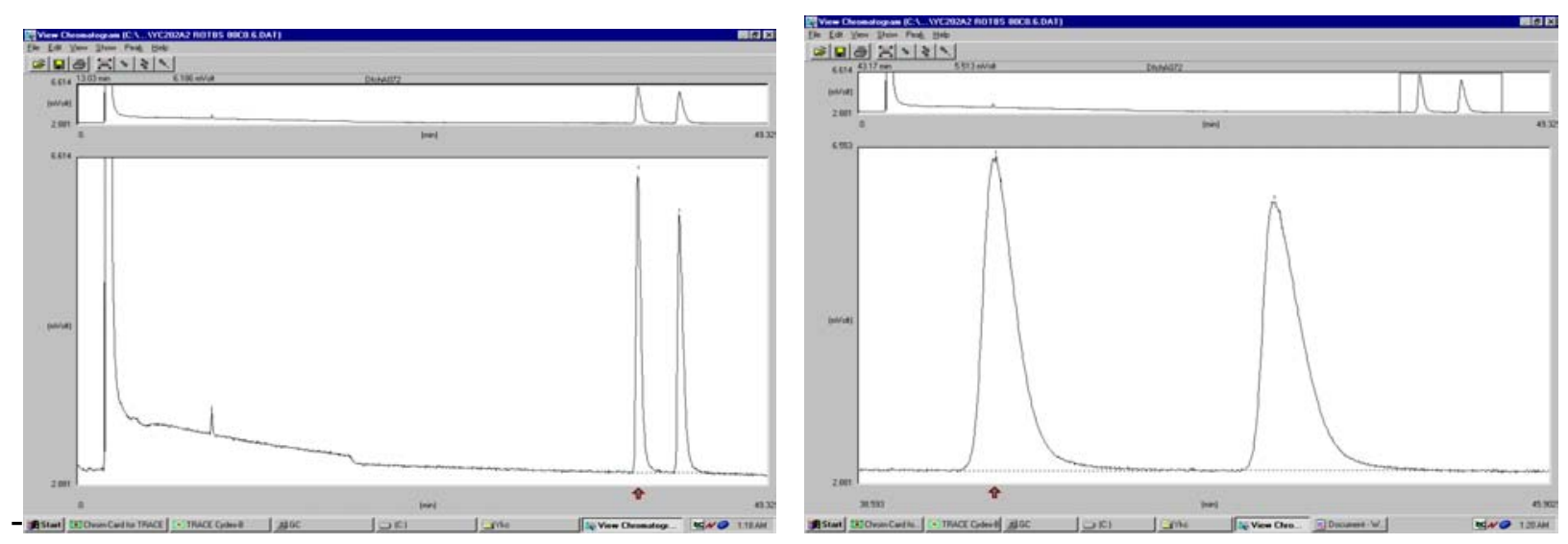

\begin{tabular}{cccc} 
Peak Number & Area \% & Ret.Time & Area BC \\
\hdashline 1 & 49.9017 & 40.04 & $840979 \mathrm{mi}$ \\
2 & 50.0983 & 42.99 & $844291 \mathrm{mi}$ \\
Totals & 100.0000 & & 1685270
\end{tabular}

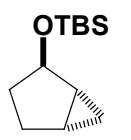

The racemic substrate was prepared according to the procedure above.

\section{3,3,6,6-Tetramethylbicyclo[3.1.0]hexan-2-ol 5b}<smiles>CCOC(=O)C(C)(C)CC=C(C)C</smiles>

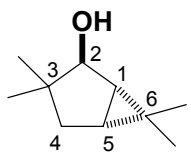

$5 b$

\section{1,2-Epoxy-3,3,6-trimethyl-5-heptene $4 \boldsymbol{b}$}

$n$-BuLi (1.6 $\mathrm{M}$ in hexane, $11.8 \mathrm{~mL}, 18.9 \mathrm{mmol}, 1.1$ equiv.) was added to a stirred solution of diisopropylamine (1.91 g, $18.9 \mathrm{mmol}, 1.1$ equiv.) in THF $(90 \mathrm{~mL})$ at $-78{ }^{\circ} \mathrm{C}$. The LDA solution formed was warmed to $\mathrm{rt}$ and then cooled to $-78{ }^{\circ} \mathrm{C}$. Ethyl isobutyrate $(2.0 \mathrm{~g}, 2.3 \mathrm{~mL}, 17.2 \mathrm{mmol})$ was added slowly to the LDA solution and the resulting mixture was stirred at the same temperature for $1 \mathrm{~h}$, and then treated with prenyl bromide $(2.1 \mathrm{~mL}, 18.1 \mathrm{mmol}, 1.05$ equiv.). After $30 \mathrm{~min}$, the mixture was warmed to $\mathrm{rt}$ and stirred for $12 \mathrm{~h}$. The reaction mixture was diluted with $\mathrm{Et}_{2} \mathrm{O}(200 \mathrm{~mL})$ and washed with water $(200 \mathrm{~mL})$. The organic layer was dried $\left(\mathrm{MgSO}_{4}\right)$, filtered and concentrated 
to give the crude product. Purification by column chromatography (5\% $\mathrm{Et}_{2} \mathrm{O}$ in petrol) gave 2,2,5,5-tetramethylpent-4-enoate $(2.75 \mathrm{~g}, 14.9 \mathrm{mmol}, 87 \%)$ as a colorless oil, $R_{\mathrm{f}}=0.4\left(5 \% \mathrm{Et}_{2} \mathrm{O}\right.$ in petrol); IR (film) 2980s, 2933s (C-H), 1733s (C=O), 1471m, 1448m, 1387m, 1364w, 1308m, $1251 \mathrm{~m}, 1181 \mathrm{~s}, 1130 \mathrm{~s}, 1032 \mathrm{~m} ;{ }^{1} \mathrm{H}$ NMR (400 MHz) $1.16\left(\mathrm{~s}, 6 \mathrm{H}, 3 \times \mathrm{H}-7,3 \times \mathrm{H}-7^{\prime}\right), 1.25(\mathrm{t}, 3 \mathrm{H}$, $\left.\mathrm{OCH}_{2} \mathrm{CH}_{3}, J=7.2\right), 1.61,1.70\left(\mathrm{~s}, 6 \mathrm{H}, 3 \times \mathrm{H}-6,3 \times \mathrm{H}^{-6}\right.$ ) $, 2.22(\mathrm{~d}, 2 \mathrm{H}, 2 \times \mathrm{H}-3, J=7.6), 4.11(\mathrm{q}, 2 \mathrm{H}$, $\left.\mathrm{OCH}_{2}, J=7.2\right), 5.06-5.10(\mathrm{~m}, 1 \mathrm{H}, \mathrm{H}-4) ;{ }^{13} \mathrm{C} \mathrm{NMR}(100 \mathrm{MHz}) 14.2\left(\mathrm{OCH}_{2} \mathrm{CH}_{3}\right), 17.9(\mathrm{C}-6), 24.8$ (C-7, 7'), $26.0\left(\mathrm{C}-6^{\prime}\right), 38.7$ (C-3), 42.7 (C-2), $60.2\left(\mathrm{OCH}_{2}\right), 119.9(\mathrm{C}-4), 134.0(\mathrm{C}-5), 177.9(\mathrm{C}-1)$.

$\mathrm{LiAlH}_{4}(2.3 \mathrm{M}$ in THF, $10.6 \mathrm{~mL}, 24.4 \mathrm{mmol}, 2.0$ equiv.) was added slowly to a stirred solution of ethyl 2,2,5,5-tetramethylpent-4-enoate $(2.25 \mathrm{~g}, 12.2 \mathrm{mmol})$ in THF $(112 \mathrm{~mL})$ at $0{ }^{\circ} \mathrm{C}$. The resulting mixture was stirred at the same temperature for $4 \mathrm{~h}$, and then quenched by sequential addition of water $(0.93 \mathrm{~mL})$, aq. $\mathrm{NaOH}(15 \%, 0.93 \mathrm{~mL})$ and water $(2.8 \mathrm{~mL})$. The solids formed were filtered and washed through with $\mathrm{Et}_{2} \mathrm{O}(100 \mathrm{~mL})$. The filtrate was dried $\left(\mathrm{MgSO}_{4}\right)$, filtered and concentrated to give crude 2,2,5,5-tetramethylpent-4-en-1-ol (1.64 g, $11.5 \mathrm{mmol}, 94 \%)$ as a colorless oil, which was sufficiently pure to be sued in the next step; IR (film) 3351bs (O-H), 2965s, 2928s, 2871s (C-H), $1472 \mathrm{~m}, 1451 \mathrm{~m}, 1378 \mathrm{~m}, 1363 \mathrm{w}, 1263 \mathrm{w}, 1186 \mathrm{w}, 1104 \mathrm{w}, 1042 \mathrm{~s} ;{ }^{1} \mathrm{H}$ NMR (400 MHz) 0.88 (s, $6 \mathrm{H}, 3 \times$ H-7, $\left.3 \times \mathrm{H}^{-7} 7^{\prime}\right), 1.63,1.73\left(2 \times \mathrm{s}, 6 \mathrm{H}, 3 \times \mathrm{H}-6,3 \times \mathrm{H}^{\prime} 6^{\prime}\right), 1.95(\mathrm{~d}, 2 \mathrm{H}, 2 \times \mathrm{H}-3, J=7.2), 3.33(\mathrm{~s}, 2 \mathrm{H}$, $2 \times \mathrm{H}-1), 5.19-5.23$ (m, 1H, H-4); ${ }^{13} \mathrm{C}$ NMR (100 MHz) 17.8 (C-6), 23.9 (C-7, 7'), $26.0\left(\mathrm{C}-6^{\prime}\right)$, 36.3 (C-2), 37.0 (C-3), 72.0 (C-1), 120.6 (C-4), 133.4 (C-5).

DMSO (1.99 mL, $28.0 \mathrm{mmol}, 2.5$ equiv.) was added dropwise to a stirred solution of oxalyl chloride (1.04 mL, $12.3 \mathrm{mmol}, 1.1$ equiv.) in $\mathrm{CH}_{2} \mathrm{Cl}_{2}(80 \mathrm{~mL})$ at $-78{ }^{\circ} \mathrm{C}$. After $5 \mathrm{~min}$, the mixture was treated slowly with 2,2,5,5-tetramethylpent-4-en-1-ol (1.60 g, $11.2 \mathrm{mmol})$, stirred for $15 \mathrm{~min}$ and then treated slowly with $\mathrm{Et}_{3} \mathrm{~N}$ (7.9 mL, $56.0 \mathrm{mmol}, 5.0$ equiv.). After $5 \mathrm{~min}$, the resulting mixture was warmed to $\mathrm{rt}$ and stirred for $3 \mathrm{~h}$. The reaction mixture was poured into water $(100 \mathrm{~mL})$, the layers were separated and then the aqueous layer extracted with $\mathrm{CH}_{2} \mathrm{Cl}_{2}(100 \mathrm{~mL})$. The combined organic layers were dried $\left(\mathrm{MgSO}_{4}\right)$, filterd and concentrated to give the crude product. Purification by column chromatography $\left(10 \% \mathrm{Et}_{2} \mathrm{O}\right.$ in petrol) gave 2,2,5,5-tetramethylpent-4-enal $(1.24 \mathrm{~g}, 8.83$ 
mmol, 79\%) as a yellow oil, $R_{\mathrm{f}}=0.5\left(10 \% \mathrm{Et}_{2} \mathrm{O}\right.$ in petrol); IR (film) $2969 \mathrm{~s}, 2930 \mathrm{~s}(\mathrm{C}-\mathrm{H}), 1725 \mathrm{~s}$ $(\mathrm{C}=\mathrm{O}), 1470 \mathrm{~m}, 1379 \mathrm{~m}, 1104 \mathrm{w}, 1080 \mathrm{w} ;{ }^{1} \mathrm{H} \mathrm{NMR}(400 \mathrm{MHz}) 1.05$ (s, 6H, $\left.3 \times \mathrm{H}-7,3 \times \mathrm{H}-7^{\prime}\right), 1.61$, $1.71\left(2 \times \mathrm{s}, 6 \mathrm{H}, 3 \times \mathrm{H}-6,3 \times \mathrm{H}-6^{\prime}\right), 2.16(\mathrm{~d}, 2 \mathrm{H}, 2 \times \mathrm{H}-3, J=8.0), 5.04-5.09(\mathrm{~m}, 1 \mathrm{H}, \mathrm{H}-4), 9.48(\mathrm{~s}$, 1H, H-1); ${ }^{13} \mathrm{C}$ NMR (100 MHz) 17.8 (C-6), 21.1 (C-7, 7'), 25.9 (C-6'), 35.5 (C-2), 118.7 (C-4), 134.7 (C-5), 206.5 (C-1).

$n$-BuLi (1.6 M in hexane, $3.3 \mathrm{~mL}, 5.2 \mathrm{mmol}, 1.05$ equiv.) was added dropwise ( $\sim 1 \mathrm{drop} / \mathrm{s})$ to a stirred solution of 2,2,5,5-tetramethylpent-4-enal $(694 \mathrm{mg}, 4.95 \mathrm{mmol})$ and dibromomethane $(0.42$ $\mathrm{mL}, 5.94 \mathrm{mmol}, 1.2$ equiv.) in THF $(20 \mathrm{~mL})$ at $-78{ }^{\circ} \mathrm{C} .^{[6]}$ The resulting mixture was warmed to $\mathrm{rt}$ and stirred for $14 \mathrm{~h}$. The reaction mixture was poured into sat. aq. $\mathrm{NH}_{4} \mathrm{Cl}(50 \mathrm{~mL})$, the layers were separated and the aqueous layer extracted with $\mathrm{Et}_{2} \mathrm{O}(50 \mathrm{~mL})$. The combined organic layers were dried $\left(\mathrm{MgSO}_{4}\right)$, filtered and concentrated to give the crude product. Purification by column chromatography $\left(10 \% \mathrm{Et}_{2} \mathrm{O}\right)$ gave epoxide $4 \mathbf{b}(425 \mathrm{mg}, 2.76 \mathrm{mmol}, 56 \%)$ as a colorless oil, $R_{\mathrm{f}}=$ $0.45\left(10 \% \mathrm{Et}_{2} \mathrm{O}\right.$ in petrol); IR (film) 3050w (epoxide), 2966s, 2929s (C-H), 1466m, 1452m, 1397w, 1379m, 1360m, 1260w, 1104w; ${ }^{1} \mathrm{H}$ NMR (400 MHz) $0.81\left(\mathrm{~s}, 3 \mathrm{H}, \mathrm{COC}\left(\mathrm{CH}_{3}\right)\right), 0.87$ (s, 3H, $\left.\mathrm{COC}\left(\mathrm{CH}_{3}\right)\right), 1.61\left(\mathrm{~s}, 3 \mathrm{H}, \mathrm{C}=\mathrm{C}\left(\mathrm{CH}_{3}\right)_{2}\right), 1.72\left(\mathrm{~s}, 3 \mathrm{H}, \mathrm{C}=\mathrm{C}\left(\mathrm{CH}_{3}\right)_{2}\right), 1.95-2.06(\mathrm{~m}, 2 \mathrm{H}, 2 \times \mathrm{H}-4), 2.59-$ $2.64\left(\mathrm{~m}, 2 \mathrm{H}, \mathrm{H}-1, \mathrm{H}-1^{\prime}\right), 2.76(\mathrm{dd}, 1 \mathrm{H}, \mathrm{H}-2, J=2.8,4.0), 5.19-5.24(\mathrm{~m}, 1 \mathrm{H}, \mathrm{H}-5) ;{ }^{13} \mathrm{C} \mathrm{NMR}(100$ MHz) $17.8\left(\mathrm{C}=\mathrm{C}\left(\mathrm{CH}_{3}\right)_{2}\right), 21.9\left(\mathrm{COC}\left(\mathrm{CH}_{3}\right)_{2}\right), 23.1\left(\mathrm{COC}\left(\mathrm{CH}_{3}\right)_{2}\right), 26.0\left(\mathrm{C}=\mathrm{C}\left(\mathrm{CH}_{3}\right)_{2}\right), 34.3(\mathrm{C}-3)$, 38.3 (C-4), 44.0 (C-1), 59.4 (C-2), 119.9 (C-5), 133.7 (C-6); MS (CI+) m/z: $172.2\left(\mathrm{MNH}_{4}^{+}\right.$5\%), $155.1\left(\mathrm{MH}^{+}\right.$6\%), 137.1 (100\%), 135.1 (23\%), 133.1 (5\%), $121.1(10 \%), 109.1$ (3\%); HRMS m/z: $\mathrm{MNH}_{4}{ }^{+}$found $172.1709, \mathrm{C}_{10} \mathrm{H}_{22} \mathrm{NO}$ requires 172.1701 .

\section{3,3,6,6-Tetramethylbicyclo[3.1.0]hexan-2-ol $\mathbf{5 b}$}

Following the typical cyclopropanation procedure, 1,2-epoxy-3,3,6-trimethyl-5-heptene $\mathbf{4 b}$ (154 mg, $1.00 \mathbf{m m o l})$ was treated with LTMP in $\mathrm{Et}_{2} \mathrm{O}$ for $8 \mathrm{~h}$ to give bicyclic alcohol $\mathbf{5 b}(116 \mathrm{mg}, 0.75 \mathrm{mmol}$, $75 \%)$ as a white solid, $R_{\mathrm{f}}=0.2\left(20 \% \mathrm{Et}_{2} \mathrm{O}\right.$ in petrol); $\mathrm{mp}=73-75{ }^{\circ} \mathrm{C}$; IR (KBr) 3306bs $(\mathrm{O}-\mathrm{H})$, 3016w (cyclopropane), 2938s (C-H), 2740s, 1456s, 1372w, 1345w, 1288w, 1266w, 1238w, 1207w, 1131w, 1095w, 1053m, 1010w; ${ }^{1} \mathrm{H}$ NMR (400 MHz) 0.95 (s, 3H, $\left.\mathrm{CH}_{3}\right), 1.00$ (s, 3H, $\mathrm{CH}_{3}$ ), 1.01 (s, 
$\left.6 \mathrm{H}, 2 \times \mathrm{CH}_{3}\right), 1.05-1.06(\mathrm{~m}, 1 \mathrm{H}, \mathrm{H}-4), 1.08-1.09(\mathrm{~m}, 1 \mathrm{H}, \mathrm{H}-2), 1.15-1.20(\mathrm{~m}, 1 \mathrm{H}, \mathrm{H}-3), 1.46(\mathrm{~s}$, $1 \mathrm{H}, \mathrm{OH}), 1.52(\mathrm{dd}, 1 \mathrm{H}, \mathrm{H}-4, J=7.6,13.6), 3.42(\mathrm{~d}, 1 \mathrm{H}, \mathrm{H}-1, J=2.0) ;{ }^{13} \mathrm{C} \mathrm{NMR}(100 \mathrm{MHz}) 14.4$ $\left(\mathrm{CH}_{3}\right), 22.0\left(\mathrm{CH}_{3}\right), 23.8(\mathrm{C}-6), 24.4\left(\mathrm{CH}_{3}\right), 27.2\left(\mathrm{CH}_{3}\right), 28.2(\mathrm{C}-3), 37.2(\mathrm{C}-2), 37.5(\mathrm{C}-4), 52.7(\mathrm{C}-$ 2), 79.3 (C-1); Elemental analysis: found $\mathrm{C}, 77.81 ; \mathrm{H}, 11.79 ; \mathrm{O}, 10.40 ; \mathrm{C}_{10} \mathrm{H}_{18} \mathrm{O}$ requires $\mathrm{C}, 77.87$; H, 11.76; O, 10.37 .

\section{5-Phenylbicyclo[3.1.0]hexan-2-ol 5c}

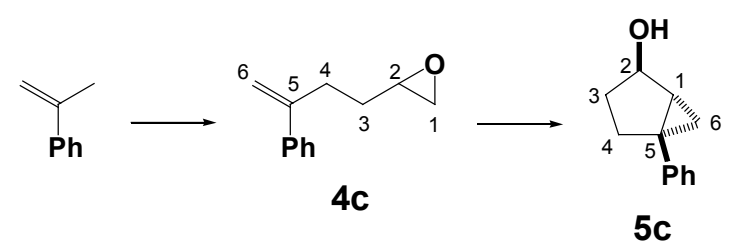

\section{1,2-Epoxy-5-phenyl-5-hexene $4 c$}

$n$-BuLi (1.6 M in hexanes, $6.25 \mathrm{~mL}, 10.0 \mathrm{mmol})$ was added dropwise to a stirred solution of $t$-BuOK $(1.12 \mathrm{~g}, 10.0 \mathrm{mmol})$ in $\mathrm{THF}(40 \mathrm{~mL})$ at $-78^{\circ} \mathrm{C}$. After $5 \mathrm{~min}, \alpha$-methylstyrene $(1.30 \mathrm{~mL}, 10.0 \mathrm{mmol})$ was added dropwise and following further stirring for $30 \mathrm{~min}$, epichlorohydrin $(0.39 \mathrm{~mL}, 5.00$ mmol) was added in one portion. Following stirring for 90 min, sat. aq. $\mathrm{NH}_{4} \mathrm{Cl}(5 \mathrm{~mL})$ and $\mathrm{Et}_{2} \mathrm{O}(5$ $\mathrm{mL})$ were added and the layers separated. The aqueous layer was washed with $\mathrm{Et}_{2} \mathrm{O}(3 \times 10 \mathrm{~mL})$, the combined organic layers were dried $\left(\mathrm{MgSO}_{4}\right)$ and concentrated under reduced pressure. Purification of the residue by column chromatography gave a crude oil. Bulb-to-bulb distillation (75 ${ }^{\circ} \mathrm{C}, 0.07$ mbar) of the crude oil gave 1,2-epoxy-5-phenyl-5-hexene $4 \mathbf{c}$ as a colorless oil (454 mg, $2.61 \mathrm{mmol}, 52 \%), R_{\mathrm{f}}=0.20\left(3 \% \mathrm{Et}_{2} \mathrm{O}\right.$ in petrol); IR (film) $3082 \mathrm{w}, 3053 \mathrm{~m}, 2987 \mathrm{~m}, 2923 \mathrm{~s}, 1627 \mathrm{~s}$ $(\mathrm{C}=\mathrm{C}), 1600 \mathrm{~m}, 1495 \mathrm{~s}, 1444 \mathrm{~m}, 1410 \mathrm{~m}, 1079 \mathrm{w} ;{ }^{1} \mathrm{H}$ NMR $(400 \mathrm{MHz}) 1.78-1.65(\mathrm{~m}, 2 \mathrm{H}, 2 \times \mathrm{H}-3)$, $2.48(\mathrm{dd}, 1 \mathrm{H}, \mathrm{H}-1, J=5.0,3.0), 2.76-2.62\left(\mathrm{~m}, 3 \mathrm{H}, \mathrm{H}-1^{\prime}, 2 \times \mathrm{H}-4\right), 2.99-2.95(1 \mathrm{H}, \mathrm{m}, \mathrm{H}-2), 5.13$ $(\mathrm{d}, 1 \mathrm{H}, \mathrm{H}-6, J=1), 5.33\left(\mathrm{~d}, 1 \mathrm{H}, \mathrm{H}-6{ }^{\prime}, J=1.0\right), 7.44-7.28(\mathrm{~m}, 5 \mathrm{H}, 5 \times \mathrm{Ar}-\mathrm{H}) ;{ }^{13} \mathrm{C} \mathrm{NMR}(100 \mathrm{MHz})$ 31.2 (C-4), 31.7 (C-3), $47.2\left(\mathrm{CH}_{2} \mathrm{O}\right), 51.9$ (C-2), 112.8 (C-6), 126.1 (Ar-C), 127.5 (2 × Ar-C), 128.4 $\left(2 \times\right.$ Ar-C), $140.1\left(\right.$ Ar-C $\left.{ }_{\text {quat }}\right), 147.5(=\mathrm{C}) ; \mathrm{MS}(\mathrm{CI}+) \mathrm{m} / z: 192\left(\mathrm{MNH}_{4}^{+}, 35\right), 175\left(\mathrm{MH}^{+}, 95\right), 157$ (100); HRMS $m / z: \mathrm{MH}^{+}$found $175.1129, \mathrm{C}_{12} \mathrm{H}_{15} \mathrm{O}$ requires 175.1123. 
5-Phenylbicyclo[3.1.0]hexan-2-ol 5c

Following the typical cyclopropanation procedure, 1,2-epoxy-5-phenyl-5-hexene 4c (174 mg, 1.00 mmol) was treated with LTMP in $t$-BuOMe for $16 \mathrm{~h}$ to give 5-phenylbicyclo[3.1.0]hexan-2-ol 5c (139 mg, $0.80 \mathrm{mmol}, 80 \%)$ as a colorless oil, $R_{\mathrm{f}}=0.2\left(30 \% \mathrm{Et}_{2} \mathrm{O}\right.$ in petrol); IR (film) $3346(\mathrm{OH})$, 3060 (cyclopropane), 3028, 2933, 1603, 1498, 1447, 1326, 1166, 1107, 1031, 755, 698; ${ }^{1} \mathrm{H}$ NMR (400 MHz) $0.76(\mathrm{t}, 1 \mathrm{H}, \mathrm{H}-6, J=4.8), 0.94$ (dd, 1H, H-6', $J=5.2,8.4), 1.51-1.61(\mathrm{~m}, 1 \mathrm{H}, \mathrm{H}-4), 1.69$ (s, 1H, OH), 1.73-1.78 (m, 2H, H-1, H-4'), 2.08 (dd, 1H, H-3, $J=8,12.4), 2.29$ (td, 1H, H-3', $J=$ 8.4, 12.1), $4.34(\mathrm{~d}, 1 \mathrm{H}, \mathrm{H}-2, J=3.6), 7.17-7.32\left(5 \mathrm{H}, \mathrm{H}\right.$ aromatic); ${ }^{13} \mathrm{C}$ NMR $(100 \mathrm{MHz}) 17.1(\mathrm{C}-6)$, 29.7 (C-3), 31.6 (C-4), 32.1 (C-5), 33.6 (C-1), 74.7 (C-2), 125.6 (Ar-C), 126.5 (Ar-C), 128.2 (Ar-C), 144.3 (Ar-C); MS (CI+) m/z: $157\left([\mathrm{M}-\mathrm{OH}]^{+}, 100\right), 173\left(\mathrm{M}-\mathrm{H}^{+}, 10\right) ; \mathrm{HMRS} m / z:[\mathrm{M}-\mathrm{OH}]^{+}$found 157.1017, $\mathrm{C}_{12} \mathrm{H}_{13}$ requires 157.1017 .

\section{3b-Methyl-2,3,3a,3b,4,7-hexahydro-1H-cyclopenta[1,3]cyclopropa[1,2]benzen-3-ol 5d}

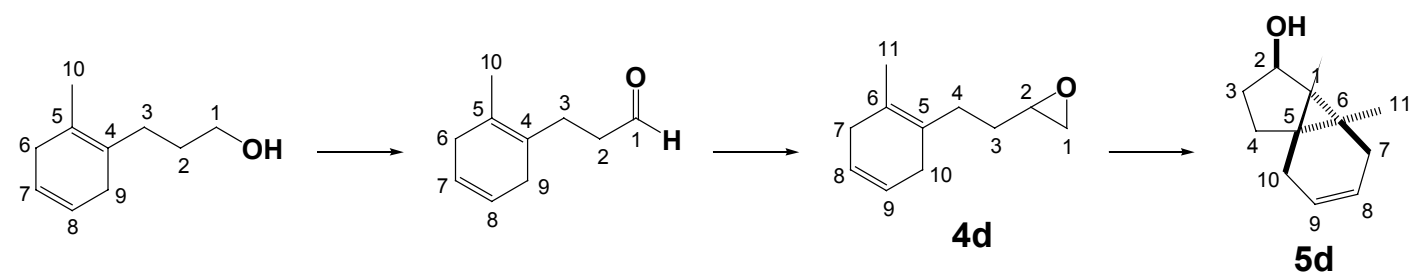

\section{4-(6-Methylcyclohexa-5,8-dienyl)-1,2-epoxybutane 4d}

$\mathrm{LiAlH}_{4}$ (2.3 M in THF, $26.2 \mathrm{~mL}, 60.3 \mathrm{mmol}, 3.0$ equiv.) was added slowly to a stirred solution of 3$o$-tolylpropionic acid $(3.30 \mathrm{~g}, 20.1 \mathrm{mmol})$ in THF $(180 \mathrm{~mL})$ at $-78{ }^{\circ} \mathrm{C}$. The resulting mixture was warmed to rt slowly over $2 \mathrm{~h}$ and then stirred at the same temperature for $4 \mathrm{~h}$. The reaction mixture was then quenched slowly by sequential addition of water $(2.3 \mathrm{~mL})$, aq. $\mathrm{NaOH}(15 \%, 2.3 \mathrm{~mL})$ and water $(6.9 \mathrm{~mL})$. The solids formed were filtered and washed through with $\mathrm{Et}_{2} \mathrm{O}(250 \mathrm{~mL})$. The filtrate was dried $\left(\mathrm{MgSO}_{4}\right)$, filtered and concentrated to give crude 3-o-tolylpropan-1-ol (3.00 g, 20.0 mmol, 100\%) as a colorless oil, which was sufficiently pure to be used without further purification.

A solution of 3-o-tolylpropan-1-ol $(2.00 \mathrm{~g}, 13.3 \mathrm{mmol})$ in THF $(20 \mathrm{~mL})$ was added slowly to a stirred dark blue solution of $\mathrm{Li}$ (554 mg, $79.8 \mathrm{mmol}, 6.0$ equiv.), $t$-BuOH (4.6 mL), THF (30 mL) in $\mathrm{NH}_{3}(80 \mathrm{~mL})$ at $-78{ }^{\circ} \mathrm{C}$. After $7 \mathrm{~h}$, the mixture was quenched slowly with $\mathrm{NH}_{4} \mathrm{Cl}$ (s) until no blue 
color was observed, then warmed to rt slowly and stirred until the excess $\mathrm{NH}_{3}$ was evaporated. The mixture was washed with water $(100 \mathrm{~mL})$, the layers were separated and then the aqueous layer extracted with $\mathrm{Et}_{2} \mathrm{O}(2 \times 60 \mathrm{~mL})$. The combined organic layers were dried $\left(\mathrm{MgSO}_{4}\right)$, filtered and concentrated to give the crude product. Purification by column chromatography $\left(60 \% \mathrm{Et}_{2} \mathrm{O}\right.$ in petrol) gave 3-(5-methylcyclohexa-4,7-dienyl)propan-1-ol (1.11 g, $7.29 \mathrm{mmol}, 55 \%)$ as a colorless oil, $R_{\mathrm{f}}=0.4\left(60 \% \mathrm{Et}_{2} \mathrm{O}\right.$ in petrol); IR (film) 3333bs $(\mathrm{O}-\mathrm{H}), 3026 \mathrm{~m}, 2927 \mathrm{~s}, 2871 \mathrm{~s}(\mathrm{C}-\mathrm{H}), 2815 \mathrm{~s}$, 1428m, 1059s; ${ }^{1} \mathrm{H}$ NMR (400 MHz) 1.51 (bs, 1H, OH), $1.63-1.70$ (m, 5H, $\left.3 \times \mathrm{H}-10,2 \times \mathrm{H}-2\right), 2.12$ $(\mathrm{t}, 2 \mathrm{H}, 2 \times \mathrm{H}-3, J=8.0), 2.59-2.66(\mathrm{~m}, 4 \mathrm{H}, 2 \times \mathrm{H}-6,2 \times \mathrm{H}-9), 3.66(\mathrm{td}, 2 \mathrm{H}, 2 \times \mathrm{H}-1, J=1.6,6.4)$, 5.66 - 5.73 (m, 2H, H-7, H-8); ${ }^{13} \mathrm{C}$ NMR (100 MHz) 18.4 (C-10), 29.0 (C-3), 30.5 (C-2), 30.9, 33.0 (C-6, C-9), 63.0 (C-1), 124.3 (C-4), 124.4 (C-7), 124.6 (C-8), 126.8 (C-5); MS (CI+) m/z: 170.2 $\left(\mathrm{MNH}_{4}{ }^{+}, 47 \%\right), 153.1\left(\mathrm{MH}^{+}, 100 \%\right), 135.1$ (61\%), $119.1(16 \%), 108.1(41 \%), 105.1$ (36\%), 91.1 (48\%); HRMS $m / z: \mathrm{MNH}_{4}^{+}$found 170.1543, $\mathrm{C}_{10} \mathrm{H}_{20} \mathrm{NO}$ requires 170.1545.

TPAP (56 mg, $0.16 \mathrm{mmol}, 5 \mathrm{~mol} \%$ ) was added to a stirred solution of 3-(5-methylcyclohexa-4,7dienyl)propan-1-ol (500 mg, $3.28 \mathrm{mmol})$, powdered $3 \AA$ Á sieves (900 mg) and 4-methylmorpholine $N$-oxide (769 mg, $6.56 \mathrm{mmol}, 2.0$ equiv.) in $\mathrm{CH}_{2} \mathrm{Cl}_{2}(25 \mathrm{~mL})$ at $\mathrm{rt}$. The resulting mixture was stirred for $4 \mathrm{~h}$, and then filtered through a thin pad of silica. The filtrate was concentrated to give the crude product. Purification by column chromatography $\left(10 \% \mathrm{Et}_{2} \mathrm{O}\right.$ in petrol) gave 3-(5-methylcyclohexa4,7-dienyl)propionaldehyde (320 mg, $2.13 \mathrm{mmol}, 65 \%)$ as a colorless oil, $R_{\mathrm{f}}=0.3\left(10 \% \mathrm{Et}_{2} \mathrm{O}\right)$; IR (film) 3028m, 2816m (C-H), 1725s (C=O), 1428m; ${ }^{1} \mathrm{H}$ NMR (400 MHz) 1.65 (s, 3H, $\left.3 \times \mathrm{H}-10\right)$, $2.36(\mathrm{t}, 2 \mathrm{H}, 2 \times \mathrm{H}-3, J=7.2), 2.51(\mathrm{td}, 2 \mathrm{H}, 2 \times \mathrm{H}-2, J=0.8,7.6), 2.60(\mathrm{~s}, 4 \mathrm{H}, 2 \times \mathrm{H}-6,2 \times \mathrm{H}-9)$, 5.65 - 5.71 (m, 2H, H-7, H-8), $9.80(\mathrm{~s}, 1 \mathrm{H}, \mathrm{CHO}) ;{ }^{13} \mathrm{C}$ NMR (100 MHz) 18.4 (C-10), 25.2 (C-3), 30.3, 32.9 (C-6, C-9), 42.2 (C-2), 124.3 (C-7), 124.4 (C-8), 125.3 (C-4), 125.4 (C-5), 202.4 (C-1); MS (CI+) m/z: $168.1\left(\left(\mathrm{MNH}_{4}^{+}, 53 \%\right), 151.2\left(\mathrm{MH}^{+}, 11 \%\right), 133.0(34 \%), 117.1(73 \%), 106.1(100 \%)\right.$; HRMS $m / z: \mathrm{MNH}_{4}{ }^{+}$found 168.1393, $\mathrm{C}_{10} \mathrm{H}_{18} \mathrm{NO}$ requires 168.1388 .

$n$-BuLi (1.6 M in hexane, $4.3 \mathrm{~mL}, 6.9 \mathrm{mmol}, 1.1$ equiv.) was added dropwise ( $1 \mathrm{drop} / 2 \mathrm{~s})$ to a stirred solution of 3-(5-methylcyclohexa-4,7-dienyl)propionaldehyde (939 mg, $6.25 \mathrm{mmol}$ ) and 
dibromomethane $(0.53 \mathrm{~mL}, 7.50 \mathrm{mmol}, 1.2$ equiv. $)$ in THF $(25 \mathrm{~mL})$ at $-78{ }^{\circ} \mathrm{C}^{[6]}$ The resulting mixture was warmed to $\mathrm{rt}$ and stirred for $20 \mathrm{~h}$. The reaction mixture was washed with sat. aq. $\mathrm{NH}_{4} \mathrm{Cl}(50 \mathrm{~mL})$, the layers were separated and then the aqueous layer extracted with $\mathrm{Et}_{2} \mathrm{O}(2 \times 40$ $\mathrm{mL})$. The combined organic layers were dried $\left(\mathrm{MgSO}_{4}\right)$, filtered and concentrated to give crude product. Purification by column chromatography (10\% $\left.\mathrm{Et}_{2} \mathrm{O}\right)$ gave epoxide $4 \mathbf{d}$ (684 mg, $4.16 \mathrm{mmol}$, $67 \%)$ as a colorless oil, $R_{\mathrm{f}}=0.4\left(10 \% \mathrm{Et}_{2} \mathrm{O}\right.$ in petrol); IR (film) $3027 \mathrm{~m}, 2921 \mathrm{~s}, 2872 \mathrm{~s}(\mathrm{C}-\mathrm{H}), 2815 \mathrm{~s}$, 1430m, 1252w, 1161w, 1011w; ${ }^{1} \mathrm{H}$ NMR (400 MHz) $1.54-1.64$ (m, 2H, $\left.2 \times \mathrm{H}-3\right), 1.66(\mathrm{~s}, 3 \mathrm{H}, 3 \times$ H-11), $2.20(\mathrm{t}, 2 \mathrm{H}, 2 \times \mathrm{H}-4, J=8.0), 2.48(\mathrm{dd}, 1 \mathrm{H}, \mathrm{H}-1, J=2.8,5.2), 2.58-2.66(\mathrm{~m}, 4 \mathrm{H}, 2 \times \mathrm{H}-7,2$ $\times \mathrm{H}-10), 2.75\left(\mathrm{dd}, 1 \mathrm{H}, \mathrm{H}-1^{\prime}, J=4.0,4.8\right), 2.90-2.94(\mathrm{~m}, 1 \mathrm{H}, \mathrm{H}-2), 5.66$ - 5.73 (m, 2H, H-8, H-9); ${ }^{13} \mathrm{C}$ NMR (100 MHz) 18.8 (C-11), 29.5 (C-4), 30.9 (C-7), 31.3 (C-3), 33.4 (C-10), 47.6 (C-1), 52.6 (C-2), 124.9, 125.0 (C-8, C-9, C-5), 126.7 (C-6); MS (CI+) m/z: $182.2\left(\mathrm{MNH}_{4}^{+}, 14 \%\right), 165.7\left(\mathrm{MH}^{+}\right.$, 53\%), 147.1 (100\%), 131.1 (66\%), 118.1 (26\%), 105.1 (63\%); HRMS $m / z: \mathrm{MH}^{+}$found 165.1287, $\mathrm{C}_{11} \mathrm{H}_{17} \mathrm{O}$ requires 165.1279 .

3b-Methyl-2,3,3a,3b,4,7-hexahydro-1H-cyclopenta[1,3]cyclopropa[1,2]benzen-3-ol 5d Following the typical cyclopropanation procedure, 4-(6-methylcyclohexa-5,8-dienyl)-1,2epoxybutane 4d (164 mg, $1.0 \mathrm{mmol})$ was treated with LTMP in $t$-BuOMe for $24 \mathrm{~h}$ to give bicyclic alcohol 5d (101 mg, $0.61 \mathrm{mmol}, 61 \%)$ as a colorless oil, $R_{\mathrm{f}}=0.3\left(60 \% \mathrm{Et}_{2} \mathrm{O}\right.$ in petrol); IR (film) 3318bs (O-H), 3026s, 2868s (C-H), 2825s, 1661w, 1434m, 1324m, 1214m, 1136s, 1056s, 1018s; ${ }^{1} \mathrm{H}$ NMR (400 MHz) 1.02 (s, 3H, $3 \times \mathrm{H}-11), 1.23$ (s, 1H, H-1), 1.59 (bs, 1H, OH), 1.72 - 1.96 (m, 4H, 2 $\times \mathrm{H}-3,2 \times \mathrm{H}-4), 2.02-2.09(\mathrm{~m}, 1 \mathrm{H}, \mathrm{H}-7), 2.32-2.41\left(\mathrm{~m}, 3 \mathrm{H}, \mathrm{H}-7^{\prime}, 2 \times \mathrm{H}-10\right), 4.21(\mathrm{~d}, 1 \mathrm{H}, \mathrm{H}-2, J=$ 5.2), 5.44 - 5.61 (m, 2H, H-8, H-9); ${ }^{13} \mathrm{C}$ NMR (100 MHz) 16.4 (C-11), 21.7 (C-6), 29.7 (C-7), 30.0 (C-4), 34.2 (C-10), 37.2 (C-1), 38.1 (C-3), 75.2 (C-2), 124.7, 125.6 (C-8, C-9); MS (FI+) m/z: 164.1 $\left(\mathrm{M}^{+}, 100 \%\right) ;$ HRMS $m / z: \mathrm{M}^{+}$found $164.1205, \mathrm{C}_{11} \mathrm{H}_{16} \mathrm{O}$ requires 164.1201. 

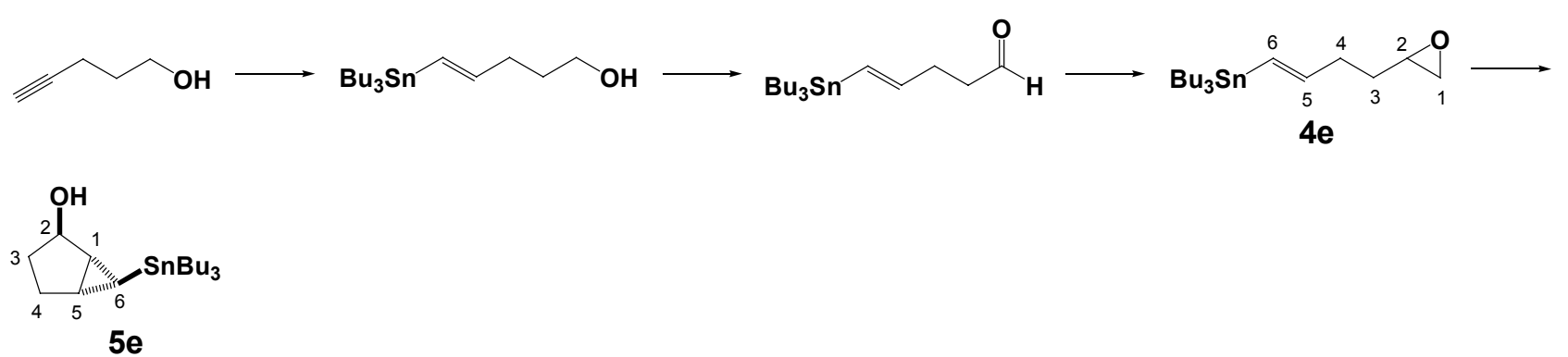

(5E)-1,2-Epoxy-6-tributylstannyl-5-hexene $4 \boldsymbol{e}$

A mixture of 4-pentyn-1-ol (2.00 g, $23.8 \mathrm{mmol})$ and AIBN (39 mg, $0.24 \mathrm{mmol}, 1 \mathrm{~mol} \%$ ) in $\mathrm{Bu}_{3} \mathrm{SnH}$ (7.0 mL, $26.2 \mathrm{mmol}, 1.1$ equiv.) was heated from $\mathrm{rt}$ to $80{ }^{\circ} \mathrm{C}$. After heating for $2 \mathrm{~h}$ at $80{ }^{\circ} \mathrm{C}$, the mixture was cooled to $\mathrm{rt}$ and chromatographed (10 - 30\% $\mathrm{Et}_{2} \mathrm{O}$ in petrol) three times to give (5E)-5tributylstannylpent-4-en-1-ol $(4.62 \mathrm{~g}, 12.3 \mathrm{mmol}, 52 \%)$ as a colorless oil, $R_{\mathrm{f}}=0.3\left(40 \% \mathrm{Et}_{2} \mathrm{O}\right.$ in petrol); ${ }^{1} \mathrm{H} \mathrm{NMR}(400 \mathrm{MHz}) 0.73-0.91\left(\mathrm{~m}, 15 \mathrm{H}, 3 \times \mathrm{Sn}\left(\mathrm{CH}_{2}\right)_{3} \mathrm{CH}_{3}, 3 \times \mathrm{Sn}\left(\mathrm{CH}_{2}\right)_{2} \mathrm{CH}_{2}\right), 1.23-1.35$ $\left(\mathrm{m}, 6 \mathrm{H}, 3 \times \mathrm{SnCH}_{2} \mathrm{CH}_{2}\right), 1.41-1.55\left(\mathrm{~m}, 6 \mathrm{H}, 3 \times \mathrm{SnCH}_{2}\right), 1.63-1.69(\mathrm{~m}, 2 \mathrm{H}, 2 \times \mathrm{H}-2), 2.13-2.21$ $(\mathrm{m}, 2 \mathrm{H}, 2 \times \mathrm{H}-3), 3.58-3.63(\mathrm{~m}, 2 \mathrm{H}, 2 \times \mathrm{H}-1), 5.83-5.95(\mathrm{~m}, 2 \mathrm{H}, \mathrm{H}-4, \mathrm{H}-5) ;{ }^{13} \mathrm{C} \mathrm{NMR}(100 \mathrm{MHz})$ $9.3\left(3 \times \mathrm{SnCH}_{2}, J\right.$ Sn-C $\left.=174\right), 13.7\left(3 \times \mathrm{Sn}\left(\mathrm{CH}_{2}\right)_{3} \mathrm{CH}_{3}\right), 27.2\left(3 \times \mathrm{Sn}\left(\mathrm{CH}_{2}\right)_{2} \mathrm{CH}_{2}\right), 29.1(3 \times$ $\mathrm{SnCH}_{2} \mathrm{CH}_{2}$ ), 31.7 (C-2), 34.1 (C-3), 62.4 (C-1), 128.1 (C-5), 148.6 (C-4).

DMSO (1.9 mL, $26.8 \mathrm{mmol}, 2.5$ equiv.) was added slowly to a stirred solution of oxalyl chloride (1.2 mL, $12.8 \mathrm{mmol}, 1.2$ equiv.) in $\mathrm{CH}_{2} \mathrm{Cl}_{2}(50 \mathrm{~mL})$ at $-78{ }^{\circ} \mathrm{C}$. After $5 \mathrm{~min}$, the mixture was treated slowly with a solution of (5E)-5-tributylstannylpent-4-en-1-ol (4.0 g, $10.7 \mathrm{mmol})$ in $\mathrm{CH}_{2} \mathrm{Cl}_{2}(10$ $\mathrm{mL})$, stirred for $15 \mathrm{~min}$ and then treated slowly with $\mathrm{Et}_{3} \mathrm{~N}(7.5 \mathrm{~mL}, 53.5 \mathrm{mmol}, 5.0$ equiv.). After 5 min, the resulting mixture was warmed to $\mathrm{rt}$ and stirred for a further $12 \mathrm{~h}$. The reaction mixture was poured into water $(100 \mathrm{~mL})$, the layers were separated and the aqueous layer extracted with $\mathrm{CH}_{2} \mathrm{Cl}_{2}$ $(2 \times 60 \mathrm{~mL})$. The combined organic layers were dried $\left(\mathrm{MgSO}_{4}\right)$, filtered and concentrated to give the crude product. Purification by column chromatography (5\% $\mathrm{Et}_{2} \mathrm{O}$ in petrol) gave (5E)-5tributylstannylpent-4-enal (1.65 g, $4.43 \mathrm{mmol}, 42 \%)$ as a light yellow oil, $R_{\mathrm{f}}=0.6\left(10 \% \mathrm{Et}_{2} \mathrm{O}\right.$ in petrol); IR (film) 2957s, 2925s (C-H), 2872m, 2852m, 1730s (C=O), 1599w, 1464w, 1417w, 1377w; ${ }^{1} \mathrm{H}$ NMR (400 MHz) $0.78-0.95\left(\mathrm{~m}, 15 \mathrm{H}, 3 \times \mathrm{Sn}\left(\mathrm{CH}_{2}\right)_{3} \mathrm{CH}_{3}, 3 \times \mathrm{Sn}\left(\mathrm{CH}_{2}\right)_{2} \mathrm{CH}_{2}\right), 1.24-1.35(\mathrm{~m}, 6 \mathrm{H}$, $\left.3 \times \mathrm{SnCH}_{2} \mathrm{CH}_{2}\right), 1.41-1.54\left(\mathrm{~m}, 6 \mathrm{H}, 3 \times \mathrm{SnCH}_{2}\right), 2.45-2.57(\mathrm{~m}, 4 \mathrm{H}, 2 \times \mathrm{H}-2,2 \times \mathrm{H}-3), 5.88-6.06$ 
$(\mathrm{m}, 2 \mathrm{H}, \mathrm{H}-4, \mathrm{H}-5), 9.78$ (t, $(1 \mathrm{H}, \mathrm{H}-1, J=1.6) ;{ }^{13} \mathrm{C} \mathrm{NMR}(100 \mathrm{MHz}) 9.4\left(3 \times \mathrm{SnCH} \mathrm{H}_{2}, J_{\mathrm{Sn}-\mathrm{C}}=171\right)$,

$13.7\left(3 \times \mathrm{Sn}\left(\mathrm{CH}_{2}\right)_{3} \mathrm{CH}_{3}\right), 27.2\left(3 \times \mathrm{Sn}\left(\mathrm{CH}_{2}\right)_{2} \mathrm{CH}_{2}\right), 29.0\left(3 \times \mathrm{SnCH}_{2} \mathrm{CH}_{2}\right), 29.9(\mathrm{C}-2), 42.7(\mathrm{C}-3)$, 129.2 (C-5), 146.3 (C-4), 202.4 (C-1).

$n$-BuLi (1.6 M in hexane, $3.1 \mathrm{~mL}, 4.9 \mathrm{mmol}, 1.1$ equiv.) was added dropwise ( $1 \mathrm{drop} / 2 \mathrm{~s})$ to a stirred solution of (5E)-5-tributylstannylpent-4-enal (1.65 g, $4.42 \mathrm{mmol})$ and dibromomethane $(0.37$ $\mathrm{mL}, 5.32 \mathrm{mmol}, 1.2$ equiv.) in $\mathrm{THF}(15 \mathrm{~mL})$ at $-78{ }^{\circ} \mathrm{C} .^{[6]}$ The resulting mixture warmed to $\mathrm{rt}$ and stirred for $16 \mathrm{~h}$. The reaction mixture was poured into sat. aq. $\mathrm{NH}_{4} \mathrm{Cl}(40 \mathrm{~mL})$, the layers were separated and then the aqueous layer extracted with $\mathrm{Et}_{2} \mathrm{O}(2 \times 40 \mathrm{~mL})$. The combined organic layers were dried $\left(\mathrm{MgSO}_{4}\right)$, filtered and concentrated to give the crude product. Purification by column chromatography $\left(10 \% \mathrm{Et}_{2} \mathrm{O}\right)$ gave epoxide $4 \mathrm{e}(475 \mathrm{mg}, 1.23 \mathrm{mmol}, 28 \%)$ as a colorless oil, $R_{\mathrm{f}}=0.4$ (10\% $\mathrm{Et}_{2} \mathrm{O}$ in petrol); IR (film) 3043w (epoxide), 2957s, 2925s (C-H), 2872m, 2853m, 1600w, 1464w, 1376w, 1340w, 1292w; ${ }^{1} \mathrm{H}$ NMR (400 MHz) $0.79-0.95\left(\mathrm{~m}, 15 \mathrm{H}, 3 \times \mathrm{Sn}\left(\mathrm{CH}_{2}\right)_{3} \mathrm{CH}_{3}, 3 \times\right.$ $\left.\mathrm{Sn}\left(\mathrm{CH}_{2}\right)_{2} \mathrm{CH}_{2}\right), 1.26-1.35\left(\mathrm{~m}, 6 \mathrm{H}, 3 \times \mathrm{SnCH}_{2} \mathrm{CH}_{2}\right), 1.41-1.55\left(\mathrm{~m}, 6 \mathrm{H}, 3 \times \mathrm{SnCH} \mathrm{H}_{2}\right), 1.61-1.70(\mathrm{~m}$, $2 \mathrm{H}, 2 \times \mathrm{H}-3), 2.23-2.38(\mathrm{~m}, 2 \mathrm{H}, 2 \times \mathrm{H}-4), 2.49(\mathrm{dd}, 1 \mathrm{H}, \mathrm{H}-1, J=2.8,5.2), 2.76\left(\mathrm{t}, 1 \mathrm{H}, \mathrm{H}-1^{\prime}, J=\right.$ 4.8), 2.92 - 2.97 (m, 1H, H-2), 5.87 - 6.06 (m, 2H, H-5, H-6); ${ }^{13} \mathrm{C}$ NMR $(100 \mathrm{MHz}) 9.4\left(3 \times \mathrm{SnCH}_{2}\right.$, $\left.J_{\text {Sn-C }}=164\right), 13.7\left(3 \times \mathrm{Sn}\left(\mathrm{CH}_{2}\right)_{3} \mathrm{CH}_{3}\right), 27.3\left(3 \times \mathrm{Sn}\left(\mathrm{CH}_{2}\right)_{2} \mathrm{CH}_{2}\right), 29.1\left(3 \times \mathrm{SnCH}_{2} \mathrm{CH}_{2}\right), 31.8(\mathrm{C}-3)$, 34.0 (C-4), 47.3 (C-1), 51.9 (C-2), 128.4 (C-6), 147.8 (C-5); ${ }^{119}$ Sn NMR (CDCl 3 , 93 MHz) -50.0; MS (CI+) m/z: $389.2\left(\mathrm{MH}^{+}, 1 \%\right), 348.1(9 \%), 308.1$ (100\%), $291.1(40 \%), 252.2(5 \%)$; HRMS m/z: $\mathrm{MH}^{+}$found 389.1852, $\mathrm{C}_{18} \mathrm{H}_{37} \mathrm{OSn}$ requires 389.1866 .

exo-6-Tributylstannylbicyclo[3.1.0] hexan-2-ol 5e

Following the typical cyclopropanation procedure, (5E)-1,2-epoxy-6-tributylstannyl-5-hexene $4 \mathbf{e}$ (300 mg, $0.77 \mathrm{mmol}$ ) was treated with LTMP in $t$-BuOMe for $16 \mathrm{~h}$ to give bicyclic alcohol $\mathbf{5 e}$ (200 $\mathrm{mg}, 0.52 \mathrm{mmol}, 68 \%)$ as a colorless oil, $R_{\mathrm{f}}=0.3\left(30 \% \mathrm{Et}_{2} \mathrm{O}\right.$ in petrol); IR (film) $3319 \mathrm{bs}(\mathrm{O}-\mathrm{H})$, 3008w, 2957s, 2925s (C-H), 2871m, 1463m, 1418m, 1377m, 1318m, 1157m; ${ }^{1} \mathrm{H}$ NMR (400 MHz)$0.52--0.45(\mathrm{~m}, 1 \mathrm{H}, \mathrm{H}-6), 0.78-0.82\left(\mathrm{~m}, 6 \mathrm{H}, 3 \times \mathrm{Sn}\left(\mathrm{CH}_{2}\right)_{2} \mathrm{CH}_{2}\right), 0.89\left(\mathrm{t}, 9 \mathrm{H}, 3 \times \mathrm{Sn}\left(\mathrm{CH}_{2}\right)_{3} \mathrm{CH}_{3}\right)$, $1.23-1.56\left(\mathrm{~m}, 17 \mathrm{H}, 3 \times \mathrm{SnCH}_{2} \mathrm{CH}_{2}, 3 \times \mathrm{SnCH}_{2}, \mathrm{OH}, \mathrm{H}-1, \mathrm{H}-5\right.$, one of cyclopentyl $\left.\mathrm{CH}_{2}\right), 1.66-$ 
$1.72\left(\mathrm{~m}, 1 \mathrm{H}\right.$, one of cyclopentyl $\left.\mathrm{CH}_{2}\right), 1.94-2.03\left(\mathrm{~m}, 1 \mathrm{H}\right.$, one of cyclopentyl $\left.\mathrm{CH}_{2}\right), 4.23-4.24(\mathrm{~m}$, $1 \mathrm{H}, \mathrm{H}-2) ;{ }^{13} \mathrm{C}$ NMR $(100 \mathrm{MHz}) 2.5(\mathrm{C}-6), 8.6\left(3 \times \mathrm{SnCH}_{2}, J\right.$ Sn-C $\left.=161\right), 13.7\left(3 \times \mathrm{Sn}\left(\mathrm{CH}_{2}\right)_{3} \mathrm{CH}_{3}\right)$, 20.7, 25.7 (one of cyclopentyl $\left.\mathrm{CH}_{2}\right), 27.3\left(3 \times \mathrm{Sn}\left(\mathrm{CH}_{2}\right)_{2} \mathrm{CH}_{2}\right), 28.7,29.1\left(3 \times \mathrm{SnCH}_{2} \mathrm{CH}_{2}\right), 30.6$, 75.5 (C-2); ${ }^{119} \mathrm{Sn}$ NMR (93 MHz) -7.6; MS (ES+) m/z: $411.2\left(\mathrm{MNa}^{+}, 54 \%\right), 388.3$ (39\%), 323.2 (47\%), 267.1 (41\%), 235.0 (19\%); HRMS m/z: $\mathrm{MNa}^{+}$found 411.1685, $\mathrm{C}_{18} \mathrm{H}_{36} \mathrm{NaOSn}$ requires 411.1680.

\section{5-Tributylstannylbicyclo[3.1.0]hexan-2-ol $5 f$}

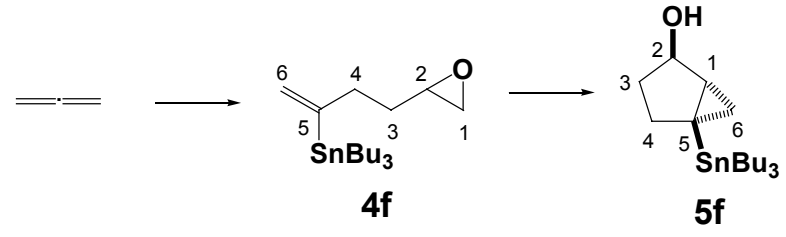

\section{1,2-Epoxy-5-tributylstannyl-5-hexene $4 f$}

A solution of $\mathrm{Bu}_{3} \mathrm{SnLi}\left(6.00 \mathrm{mmol}, 2.0\right.$ equiv.) [prepared by addition of $\mathrm{Bu}_{3} \mathrm{SnH}(1.61 \mathrm{~mL}, 6.00$ mmol) to a stirred solution of LDA $(6.00 \mathrm{mmol})$ in THF $(12 \mathrm{~mL})$ at $0{ }^{\circ} \mathrm{C}$, and then stirred for $5 \mathrm{~min}$ ] was added by syringe to a stirred suspension of $\mathrm{CuCN}(269 \mathrm{mg}, 3.00 \mathrm{mmol})$ in THF (10 mL) at -78 ${ }^{\circ} \mathrm{C} .{ }^{[7]}$ The resulting brown solution was stirred at the same temperature for $30 \mathrm{~min}$, and then treated with allene $\left(\sim 200 \mathrm{~cm}^{3}\right.$ in a balloon, $\sim 3.30 \mathrm{mmol}, \sim 1.1$ equiv.). Completion of addition was indicated by deflation of the balloon. The resulting orange/brown stannyl-cuprate solution was stirred at -78 ${ }^{\circ} \mathrm{C}$ for $30 \mathrm{~min}$ then treated with epichlorohydrin $(0.94 \mathrm{~mL}, 12.0 \mathrm{mmol}, 4.0$ equiv.). After $1 \mathrm{~h}$, the mixture was warmed to $0{ }^{\circ} \mathrm{C}$ and stirred for a further $5 \mathrm{~h}$. The reaction mixture was poured into sat. aq. $\mathrm{NH}_{4} \mathrm{Cl}(50 \mathrm{~mL})$, the layers were separated and then the aqueous layer extracted with $\mathrm{Et}_{2} \mathrm{O}(2 \times$ $50 \mathrm{~mL})$. The combined organic layers were dried $\left(\mathrm{MgSO}_{4}\right)$, filtered and concentrated to give the crude product. Purification by column chromatography (10\% $\mathrm{Et}_{2} \mathrm{O}$ in petrol) gave epoxide $4 \mathbf{f}$ (673 $\mathrm{mg}, 1.74 \mathrm{mmol}, 29 \%$ ) as a colorless oil, $R_{\mathrm{f}}=0.4\left(10 \% \mathrm{Et}_{2} \mathrm{O}\right.$ in petrol); IR (film) $3040 \mathrm{w}$ (epoxide), 2957s, 2927s, 2872s (C-H), 1464m, 1377w, 1340w, 1261w, 1072m; ${ }^{1} \mathrm{H}$ NMR (400 MHz) $0.82-0.98$ $\left(\mathrm{m}, 15 \mathrm{H}, 3 \times \mathrm{Sn}\left(\mathrm{CH}_{2}\right)_{3} \mathrm{CH}_{3}, 3 \times \mathrm{Sn}\left(\mathrm{CH}_{2}\right)_{2} \mathrm{CH}_{2}\right), 1.28-1.37\left(\mathrm{~m}, 6 \mathrm{H}, 3 \times \mathrm{SnCH}_{2} \mathrm{CH}_{2}\right), 1.44-1.53(\mathrm{~m}$, $\left.6 \mathrm{H}, 3 \times \mathrm{SnCH}_{2}\right), 1.59-1.66(\mathrm{~m}, 2 \mathrm{H}, 2 \times \mathrm{H}-3), 2.34-2.47(\mathrm{~m}, 2 \mathrm{H}, 2 \times \mathrm{H}-4), 2.49(\mathrm{dd}, 1 \mathrm{H}, \mathrm{H}-1, J=$ 2.4, 4.8), 2.77 (t, 1H, H-1', $J=4.0), 2.91-2.96(\mathrm{~m}, 1 \mathrm{H}, \mathrm{H}-2), 5.15(\mathrm{~d}, 1 \mathrm{H}, \mathrm{H}-6, J=2.4), 5.73(\mathrm{~s}, 1 \mathrm{H}$, 
H-6 $) ;{ }^{13} \mathrm{C}$ NMR $(100 \mathrm{MHz}) 9.5\left(3 \times \mathrm{SnCH} 2, J_{\mathrm{Sn}-\mathrm{C}}=166\right), 13.7\left(3 \times \mathrm{Sn}\left(\mathrm{CH}_{2}\right)_{3} \mathrm{CH}_{3}\right), 27.4(3 \times$ $\left.\mathrm{Sn}\left(\mathrm{CH}_{2}\right)_{2} \mathrm{CH}_{2}\right), 29.1\left(3 \times \mathrm{SnCH}_{2} \mathrm{CH}_{2}\right), 32.4(\mathrm{C}-3), 37.1(\mathrm{C}-4), 47.3(\mathrm{C}-1), 51.9(\mathrm{C}-2), 125.3(\mathrm{C}-6)$, 154.00 (C-5); ${ }^{119} \mathrm{Sn}$ NMR (93 MHz) -44.9; MS (CI-) m/z: $387.3\left([\mathrm{M}-\mathrm{H}]^{-}, 46 \%\right), 361.1$ (70\%), 313.1 (100\%), 291.2 (70\%), 269.1 (30\%), 218.2 (22\%); HRMS $m / z:[\mathrm{M}-\mathrm{H}]^{-}$found 387.1713, $\mathrm{C}_{18} \mathrm{H}_{35} \mathrm{O}^{120} \mathrm{Sn}$ requires 387.1715 .

\section{5-Tributylstannylbicyclo[3.1.0]hexan-2-ol 5f}

Following the typical cyclopropanation procedure, 1,2-epoxy-5-tributylstannyl-5-hexene 4 ff (387 $\mathrm{mg}, 1.00 \mathrm{mmol}$ ) was treated with LTMP in $t$-BuOMe for $24 \mathrm{~h}$ to give bicyclic alcohol $\mathbf{5 f}$ ( $256 \mathrm{mg}$, $0.66 \mathrm{mmol}, 66 \%)$ as a colorless oil; $R_{\mathrm{f}}=0.3\left(20 \% \mathrm{Et}_{2} \mathrm{O}\right.$ in petrol); IR (film) $3345 \mathrm{bs}(\mathrm{O}-\mathrm{H}), 2956 \mathrm{~s}$, 2925s, 2872s (C-H), 1464s, 1418m, 1376s, 1340m, 1292m, 1250m, 1212m, 1185m, 1058m; ${ }^{1} \mathrm{H}$ $\operatorname{NMR}(500 \mathrm{MHz}) 0.18(\mathrm{dd}, 1 \mathrm{H}, \mathrm{H}-6, J=3.5,4.5), 0.36\left(\mathrm{dd}, 1 \mathrm{H}, \mathrm{H}-6^{\prime}, J=5.5,7.5\right), 0.84(\mathrm{t}, 6 \mathrm{H}, 3 \times$ $\left.\mathrm{Sn}\left(\mathrm{CH}_{2}\right)_{2} \mathrm{CH}_{2}, J=8.5\right), 0.90\left(\mathrm{t}, 9 \mathrm{H}, 3 \times \mathrm{Sn}\left(\mathrm{CH}_{2}\right)_{3} \mathrm{CH}_{3}, J=7.5\right), 1.24(\mathrm{~d}, 1 \mathrm{H}, \mathrm{OH}, J=6.0), 1.28-$ $1.54\left(\mathrm{~m}, 15 \mathrm{H}, 3 \times \mathrm{SnCH}_{2}, 3 \times \mathrm{SnCH}_{2} \mathrm{CH}_{2}, \mathrm{H}-1,2 \times \mathrm{H}-3\right), 1.76(\mathrm{dd}, 1 \mathrm{H}, \mathrm{H}-4, J=8.5,12.5), 1.86-$ $1.92\left(\mathrm{~m}, 1 \mathrm{H}, \mathrm{H}-4^{\prime}\right), 4.35(\mathrm{t}, 1 \mathrm{H}, \mathrm{H}-2, J=5.5) ;{ }^{13} \mathrm{C} \mathrm{NMR}(100 \mathrm{MHz}) 8.5\left(3 \times \mathrm{SnCH} 2, J_{\mathrm{Sn}-\mathrm{C}}=158\right)$, $11.1(\mathrm{C}-6), 12.1(\mathrm{C}-5), 13.7\left(3 \times \mathrm{Sn}\left(\mathrm{CH}_{2}\right)_{3} \mathrm{CH}_{3}\right), 27.4\left(3 \times \mathrm{Sn}\left(\mathrm{CH}_{2}\right)_{2} \mathrm{CH}_{2}\right), 29.1\left(3 \times \mathrm{SnCH}_{2} \mathrm{CH}_{2}\right)$, 29.2 (C-1), 30.2 (C-3), 30.3 (C-4), 75.1 (C-2); ${ }^{119}$ Sn NMR (125 MHz) -10.0; MS (CI-, $\left.{ }^{120} \mathrm{Sn}\right) \mathrm{m} / z$ : $387.3\left([\mathrm{M}-\mathrm{H}]^{-}, 100 \%\right), 361.1(65 \%), 313.1(100 \%), 291.2(15 \%), 269.1(30 \%)$; HRMS m/z: [M-H] found $387.1718, \mathrm{C}_{18} \mathrm{H}_{35} \mathrm{O}^{120} \mathrm{Sn}$ requires 387.1715 . 

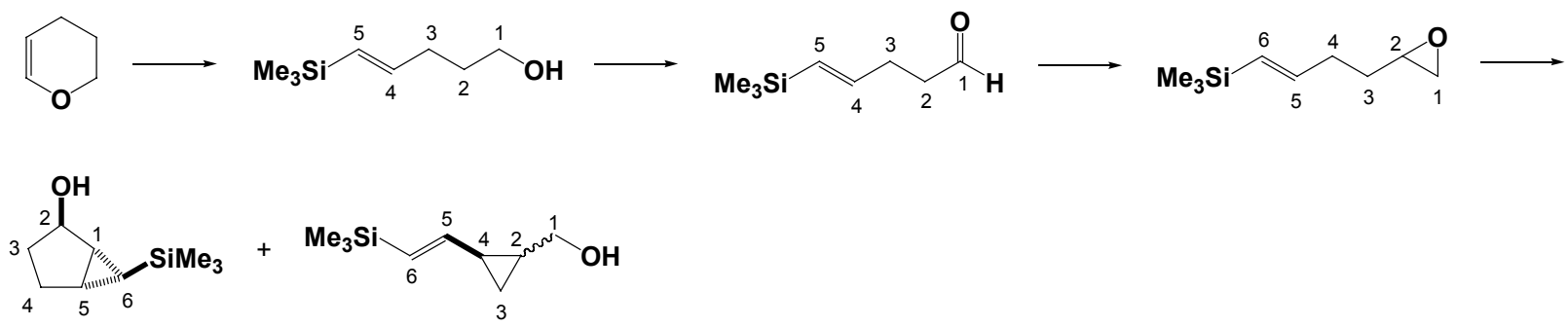

(5E)-Trimethylsilylpent-4-en-1-ol ${ }^{[8]}$

SOLUTION A: $t$-BuLi (1.7 M in pentane, $7.1 \mathrm{~mL}, 12 \mathrm{mmol})$ was added slowly to a stirred solution of freshly distilled 3,4-dihydro- $2 H$-pyran $(0.841 \mathrm{~g}, 10.0 \mathrm{mmol})$ in THF $(8 \mathrm{~mL})$ at $-60{ }^{\circ} \mathrm{C}$. After 10 min, the mixture was placed in an ice-bath for $50 \mathrm{~min}$.

SOLUTION B: MeLi (1.6 $\mathrm{M}$ in $\left.\mathrm{Et}_{2} \mathrm{O}, 13.3 \mathrm{~mL}, 21 \mathrm{mmol}\right)$ was added to a stirred solution of hexamethyldisilane $(3.28 \mathrm{~g}, 22.4 \mathrm{mmol})$ and HMPA $(4.12 \mathrm{~g}, 23.0 \mathrm{mmol})$ in THF $(20 \mathrm{~mL})$ at $0{ }^{\circ} \mathrm{C}$. After $15 \mathrm{~min}$, this mixture was added via cannula to a stirred suspension of $\mathrm{CuCN}$ (896 mg, 10.0 $\mathrm{mmol})$ in THF $(24 \mathrm{~mL})$ at $-40{ }^{\circ} \mathrm{C}$. The resulting mixture was stirred at $-25^{\circ} \mathrm{C}$ for $30 \mathrm{~min}$.

Solution A [diluted with THF $(8 \mathrm{~mL})$ ] was added via cannula to solution B [diluted with THF (28 $\mathrm{mL})]$ at $-30{ }^{\circ} \mathrm{C}$. The reaction mixture was subsequently stirred for $1.5 \mathrm{~h}$ at $-5{ }^{\circ} \mathrm{C}$, cooled to $-40{ }^{\circ} \mathrm{C}$ and then treated with $\mathrm{NH}_{4} \mathrm{Cl}(4.79 \mathrm{~g}, 89.6 \mathrm{mmol})$ in one portion. The mixture was allowed to reach rt overnight, poured into a solution of sat. aq. $\mathrm{NH}_{4} \mathrm{Cl}: \mathrm{NH}_{3}(4: 1)$ at $0{ }^{\circ} \mathrm{C}$, stirred for $30 \mathrm{~min}$, and then extracted with $\mathrm{Et}_{2} \mathrm{O}(3 \times 50 \mathrm{~mL})$. The combined organic layers were dried $\left(\mathrm{MgSO}_{4}\right)$ and concentrated to give the crude product. Purification by column chromatography $\left(50 \% \mathrm{Et}_{2} \mathrm{O}\right.$ in petrol) gave (5E)-trimethylsilylpent-4-en-1-ol as a colorless oil $(1.423 \mathrm{~g}, 8.99 \mathrm{mmol}, 90 \%), R_{\mathrm{f}}=$ $0.22\left(50 \% \mathrm{Et}_{2} \mathrm{O}\right.$ in petrol); IR (film) 3400s, 2954s, 1640m, 1247s; ${ }^{1} \mathrm{H}$ NMR (400 MHz) 0.05 (s, 9H, $\left.\mathrm{Si}\left(\mathrm{CH}_{3}\right)_{3}\right), 1.36(\mathrm{~s}, 1 \mathrm{H}, \mathrm{OH}), 1.65-1.72(\mathrm{~m}, 2 \mathrm{H}, 2 \times \mathrm{H}-2), 2.18-2.29(\mathrm{~m}, 2 \mathrm{H}, 2 \times \mathrm{H}-3), 3.66(\mathrm{t}, 2 \mathrm{H}$, $2 \times \mathrm{H}-1, J=6.8), 5.68(\mathrm{~d}, 1 \mathrm{H}, \mathrm{H}-5, J=18.4), 6.01-6.08(\mathrm{~m}, 1 \mathrm{H}, \mathrm{H}-4) ;{ }^{13} \mathrm{C} \mathrm{NMR}(100 \mathrm{MHz})-1.2$ $\left(\mathrm{Si}\left(\mathrm{CH}_{3}\right)_{3}\right), 31.5(\mathrm{C}-3), 32.9(\mathrm{C}-2), 62.5(\mathrm{C}-1), 130.5$ (C-5), 146.2 (C-4); MS (CI+) m/z: 176.1 $\left(\mathrm{MNH}_{4}^{+}, 35 \%\right), 159.1\left(\mathrm{MH}^{+}, 100 \%\right), 103.1(20 \%)$. 
(5E)-Trimethylsilyl-pent-4-enal

DMSO (1.56 g, $19.91 \mathrm{mmol})$ was added slowly to a stirred solution of oxalyl chloride $(1.21 \mathrm{~g}, 9.51$ mmol) in $\mathrm{CH}_{2} \mathrm{Cl}_{2}(40 \mathrm{~mL})$ at $-78{ }^{\circ} \mathrm{C}$. After $5 \mathrm{~min}$, a solution of 5(E)-trimethylsilylpent-4-en-1-ol (1.26 g, $7.95 \mathrm{mmol})$ in $\mathrm{CH}_{2} \mathrm{Cl}_{2}(10 \mathrm{~mL})$ was added dropwise. After $15 \mathrm{~min} \mathrm{Et}_{3} \mathrm{~N}(4.03 \mathrm{~g}, 39.81$ mmol) was added slowly, and then after 5 min the reaction mixture was warmed to $\mathrm{rt}$ and stirred overnight. The reaction mixture was poured into water $(100 \mathrm{~mL})$ and extracted with $\mathrm{CH}_{2} \mathrm{Cl}_{2}(2 \times$ $100 \mathrm{~mL})$. The combined organic layers were dried $\left(\mathrm{MgSO}_{4}\right)$ and concentrated in vacuo. Purification of the crude product by column chromatography (20\% $\mathrm{Et}_{2} \mathrm{O}$ in petrol) gave $(5 E)$ trimethylsilyl-pent-4-enal $(1.240 \mathrm{~g}, 7.93 \mathrm{mmol}, 99 \%)$ as a colorless oil, $R_{\mathrm{f}}=0.5\left(20 \% \mathrm{Et}_{2} \mathrm{O}\right.$ in petrol); IR (film) 2959s, 2821w, 2720w, 1728s, 1618m, 1411w, 1248s; ${ }^{1} \mathrm{H}$ NMR (400 MHz) 0.03 (s, 9H, $\left.\mathrm{Si}\left(\mathrm{CH}_{3}\right)_{3}\right), 2.45-2.58(\mathrm{~m}, 4 \mathrm{H}, 2 \times \mathrm{H}-2,2 \times \mathrm{H}-3), 5.66-5.71(\mathrm{~m}, 1 \mathrm{H}, \mathrm{H}-5), 5.99-6.06(\mathrm{~m}, 1 \mathrm{H}$, H-4), 9.78 (s, 1H, H-1); ${ }^{13} \mathrm{C}$ NMR (100 MHz) -1.3 (Si(CH$\left.)_{3}\right), 28.7$ (C-3), 42.5 (C-2), 131.3 (C-5), 144.0 (C-4), $202.1(\mathrm{C}-1)$; $\mathrm{MS}(\mathrm{CI}+) \mathrm{m} / z: 174.2\left(\mathrm{MNH}_{4}{ }^{+}, 100 \%\right), 157.1\left(\mathrm{MH}^{+}, 90 \%\right), 113.1(20 \%)$.

(5E)-1,2-Epoxy-6-trimethylsilyl-5-hexene $4 \boldsymbol{e}\left(\mathrm{SnBu}_{3}=\mathrm{SiMe}_{3}\right)$

$n$-BuLi (1.6 M solution in hexane, $1.5 \mathrm{~mL}, 2.34 \mathrm{mmol})$ was added over $1.5 \mathrm{~h}$ via a syringe pump to a solution of (5E)-trimethylsilyl-pent-4-enal (350 mg, $2.24 \mathrm{mmol})$ and dibromomethane (443 mg, 2.55 mmol) in THF $(10 \mathrm{~mL})-78{ }^{\circ} \mathrm{C}$. The resulting solution was warmed to $\mathrm{rt}$ and stirred overnight. The reaction mixture was washed with sat. aq. $\mathrm{NH}_{4} \mathrm{Cl}(50 \mathrm{~mL})$, the organic phase was dried $\left(\mathrm{MgSO}_{4}\right)$ and then concentrated in vacuo. Purification of the crude product by column chromatography (20\% $\mathrm{Et}_{2} \mathrm{O}$ in petrol) gave (5E)-1,2-epoxy-6-trimethylsilyl-5-hexene $4 \mathbf{e}\left(\mathrm{SnBu}_{3}=\mathrm{SiMe}_{3}\right)(267 \mathrm{mg}, 1.57$ mmol, $70 \%), R_{\mathrm{f}}=0.65\left(20 \% \mathrm{Et}_{2} \mathrm{O}\right.$ in petrol); IR (film) $3000 \mathrm{w}, 2955 \mathrm{~m}, 1738 \mathrm{w}, 1618 \mathrm{~m}, 1410 \mathrm{w}$, 1248s; ${ }^{1} \mathrm{H}$ NMR (400 MHz) 0.05 (s, 9H, Si(CH3) $)$, 1.61 - 1.68 (m, 2H, $\left.2 \times \mathrm{H}-4\right), 2.24$ - 2.32 (m, 2H, $2 \times \mathrm{H}-3), 2.48(\mathrm{dd}, 1 \mathrm{H}, \mathrm{H}-1, J=3.2,5.0), 2.75-2.77\left(\mathrm{~m}, 1 \mathrm{H}, \mathrm{H}-1^{\prime}\right), 2.91-2.96(\mathrm{~m}, 1 \mathrm{H}, \mathrm{H}-2), 5.70$ $(\mathrm{d}, 1 \mathrm{H}, \mathrm{H}-6, J=18.4), 6.01-6.08(\mathrm{~m}, 1 \mathrm{H}, \mathrm{H}-5) ;{ }^{13} \mathrm{C} \mathrm{NMR}(100 \mathrm{MHz})-1.2\left(\mathrm{Si}\left(\mathrm{CH}_{3}\right)_{3}\right), 31.6(\mathrm{C}-4)$, 32.8 (C-3), 47.2 (C-1), 51.9 (C-2), 130.8 (C-6), 145.5 (C-5); MS (CI+) $m / z: 188.2\left(\mathrm{MNH}_{4}^{+}, 95 \%\right.$, $171.2\left(\mathrm{MH}^{+}, 100 \%\right), 164.2(15 \%), 155.2(50 \%), 129.1(50 \%), 103.1(55 \%)$. 
6-(Trimethylsilyl)-bicyclo[3.1.0]hexan-1-ol $5 \boldsymbol{e}\left(\mathrm{SnBu}_{3}=\mathrm{SiMe}_{3}\right)$ and 2-(2-trimethylsilylvinyl)cyclopropyl-methanol $9\left(R=\right.$ SiMe $\left._{3}\right)$

Following the typical cyclopropanation procedure, (5E)-1,2-epoxy-6-trimethylsilyl-5-hexene (390 $\mathrm{mg}, 2.29 \mathrm{mmol}$ ) was treated with LTMP for $16 \mathrm{~h}$ to give an inseparable mixture of 6(trimethylsilyl)-bicyclo[3.1.0]hexan-1-ol $\mathbf{5 e}\left(\mathrm{SnBu}_{3}=\mathrm{SiMe}_{3}\right)$ and 2-(2-trimethylsilylvinyl)cyclopropyl-methanol $9\left(\mathrm{R}=\mathrm{SiMe}_{3}\right)$ as colorless oils $(273 \mathrm{mg}, 1.60 \mathrm{mmol}, 70 \%)$, in a ratio of 5:2 (determined by integration of olefin signals and $\mathrm{CHSiMe}_{3}$ ). Separation of their tert-butyldiphenyl silyl ether derivatives was achieved, see below.

\section{2-(tert-Butyldiphenylsilyloxy)-6-trimethylsilyl-bicyclo[3.1.0]hexane}

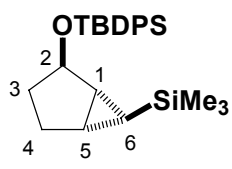

TBDPSCl (0.52 g, $1.91 \mathrm{mmol})$ was added portionwise to a stirred solution of a mixture of $5 \mathbf{e}\left(\mathrm{SnBu}_{3}=\mathrm{SiMe}_{3}\right)$ and $9\left(\mathrm{R}=\mathrm{SiMe}_{3}\right)($ in ratio 5:2) $(270 \mathrm{mg}, 1.59 \mathrm{mmol})$ and imidazole $(0.27 \mathrm{~g}, 3.98 \mathrm{mmol})$ in DMF $(2 \mathrm{~mL})$ at $0{ }^{\circ} \mathrm{C}$. Following stirring for $1 \mathrm{~h}$ the solution was warmed to rt and then stirred overnight. The reaction mixture was dry-loaded onto silica and then chromatographed (petrol) to give 2-(tert-butyldiphenylsilyloxy)-6-trimethylsilylbicyclo[3.1.0]hexane (464 mg, $1.14 \mathrm{mmol}, 100 \%$ ) as a colorless oil as the first eluted compound, $R_{\mathrm{f}}$ $=0.2$ (petrol); IR (film) 3071m, 3016m, 2956s, 2895m, 2859s, 1437m, 1428s, 1361w, 1248s, 1164s, $1111 \mathrm{~s}, 1071 \mathrm{~s}, 1026 \mathrm{~s} ;{ }^{1} \mathrm{H}$ NMR (400 MHz) -0.77 - -0.76 (m, 1H, H-6), -0.07 (s, 9H, $\left.\mathrm{Si}\left(\mathrm{CH}_{3}\right)_{3}\right), 1.19$ - $1.23\left(\mathrm{~m}, 10 \mathrm{H}, \mathrm{H}-1, \mathrm{SiC}\left(\mathrm{CH}_{3}\right)_{3}\right), 1.40-1.45(\mathrm{~m}, 2 \mathrm{H}, 2 \times \mathrm{H}-3), 1.64-1.72(\mathrm{~m}, 2 \mathrm{H}, 2 \times \mathrm{H}-4), 2.13-$ $2.21(\mathrm{~m}, 1 \mathrm{H}, 2 \times \mathrm{H}-5), 4.39-4.40(\mathrm{~m}, 1 \mathrm{H}, \mathrm{H}-2), 7.42-7.51(\mathrm{~m}, 6 \mathrm{H}, 6 \times \mathrm{Ar}-\mathrm{H}), 7.76-7.82(\mathrm{~m}, 4 \mathrm{H}, 4$ $\times \mathrm{Ar}-\mathrm{H}) ;{ }^{13} \mathrm{C} \mathrm{NMR}(100 \mathrm{MHz})-2.1\left(\mathrm{Si}\left(\mathrm{CH}_{3}\right)_{3}, 6.3(\mathrm{C}-5), 19.3\left(\mathrm{SiC}\left(\mathrm{CH}_{3}\right)_{3}\right), 21.4(\mathrm{C}-6), 26.0(\mathrm{C}-4)\right.$, $27.1\left(\mathrm{SiC}\left(\mathrm{CH}_{3}\right)_{3}\right), 28.6(\mathrm{C}-1), 31.1(\mathrm{C}-3), 76.9(\mathrm{C}-2), 127.5(2 \times \mathrm{Ar}-\mathrm{C}), 129.5(2 \times \mathrm{Ar}-\mathrm{C}), 134.9(2$ $\times$ Ar-C), $135.1(2 \times$ Ar-C), $135.8(2 \times$ Ar-C), $135.9(2 \times$ Ar-C); MS (CI+) m/z: $409.4(\mathrm{MH}+, 60 \%)$, $368.3(100 \%), 351.3(80 \%), 331.3(25 \%), 288.2$ (65\%), $268.3(80 \%), 216.2(90 \%), 170.29(90 \%)$, $153.1(90 \%), 135.1(30 \%)$. 

trimethylsilylvinyl-cyclopropylmethoxy)-diphenylsilane $(0.185 \mathrm{~g}, 0.45 \mathrm{mmol}, 100 \%)$ (cis:trans $=$ 1:7.8) as a colorless oil, $R_{\mathrm{f}}=0.05$ (petrol); IR (film) $3071 \mathrm{~m}, 3000 \mathrm{~s}, 2955 \mathrm{~s}, 2895 \mathrm{~s}, 2859 \mathrm{~s}, 1613 \mathrm{~s}$, 1473m, 1428m, 1247s, 1112s; ${ }^{1} \mathrm{H}$ NMR (400 MHz) 0.05 (s, 9H, $\left.\mathrm{Si}\left(\mathrm{CH}_{3}\right)_{3}\right), 0.60-0.71(\mathrm{~m}, 2 \mathrm{H}, 2$ $\times \mathrm{H}-3), 0.84-0.91(\mathrm{~m}, 1 \mathrm{H}, \mathrm{H}-2), 1.05\left(\mathrm{~s}, 9 \mathrm{H}, \mathrm{SiC}\left(\mathrm{CH}_{3}\right)_{3}\right), 1.30-1.38(\mathrm{~m}, 1 \mathrm{H}, \mathrm{H}-4), 3.48$ - $3.52(\mathrm{~m}$, $2 \mathrm{H}, 2 \times \mathrm{H}-1), 5.49-5.56(\mathrm{~m}, 1 \mathrm{H}, \mathrm{H}-5), 5.62$ - $5.80(\mathrm{~m}, 1 \mathrm{H}, \mathrm{H}-6), 7.37$ - $7.45(\mathrm{~m}, 6 \mathrm{H}, 6 \times \mathrm{Ar}-\mathrm{H}), 7.61$

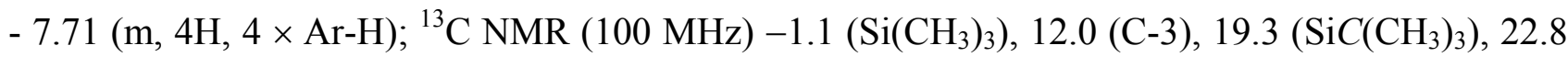
(C-2), $23.1(\mathrm{C}-4), 26.9\left(\mathrm{SiC}\left(\mathrm{CH}_{3}\right)_{3}\right), 66.1(\mathrm{C}-1), 126.8$ (4 × Ar-C), 127.6 (Ar-C), $129.5(\mathrm{C}-6), 134.0$ (Ar-C), $135.6\left(4 \times\right.$ Ar-C), $149.3(\mathrm{C}-5) ; \mathrm{MS}(\mathrm{CI}+) \mathrm{m} / z: 409.2\left(\mathrm{MH}^{+}, 30 \%\right), 297.2$ (15\%), 245.1 (15\%), $173.1(35 \%)$.

\section{4,4,5,5-Tetramethyl-2-(4-oxiranylbut-1-enyl)[1,3,2]dioxaborolane $4 e\left(\mathrm{SnBu}_{3}=\mathrm{B}\left(\mathrm{OCMe}_{2}\right)_{2}\right)$}

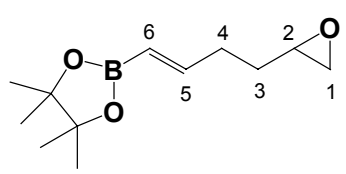

A solution of Grubbs $2^{\text {nd }}$ generation catalyst (42 mg, 5 mol\%), 1,2-epoxy-5hexene 4a (196 mg, 2.0 mmol, 2.0 equiv.) and 4,4,5,5-tetramethyl-2-vinyl-[1,3,2]dioxaborolane (154 $\mathrm{mg}, 1.00 \mathrm{mmol})$ in $\mathrm{CH}_{2} \mathrm{Cl}_{2}(5 \mathrm{~mL})$ was heated under reflux at $45{ }^{\circ} \mathrm{C}$ for $12 \mathrm{~h}$. The solvent was evaporated and the residue was purified by column chromatography ( $20 \% \mathrm{Et}_{2} \mathrm{O}$ in petrol) to give the title epoxide (168 mg, $0.75 \mathrm{mmol}, 75 \%)$ as a brown oil, $R_{\mathrm{f}}=0.2\left(20 \% \mathrm{Et}_{2} \mathrm{O}\right.$ in petrol); IR (film) 2979s, 2931s (C-H), 1640s, 1446m, 1363s, 1265m, 1146s; ${ }^{1} \mathrm{H}$ NMR (400 MHz) 1.26 (s, 12H, $4 \times$ $\left.\mathrm{CH}_{3}\right), 1.64-1.70(\mathrm{~m}, 2 \mathrm{H}, 2 \times \mathrm{H}-4), 2.26-2.37(\mathrm{~m}, 2 \mathrm{H}, 2 \times \mathrm{H}-3), 2.48(\mathrm{dd}, 1 \mathrm{H}, \mathrm{H}-1, J=2.8,4.8)$, 2.75 (dd, 1H, H-1', J = 4.4, 4.8), 2.92 - 2.96 (m, 1H, H-2), 5.48 (dt, 1H, H-6, J = 1.6, 18.0), 6.64 (dt, 1H, H-5, $J=6.0,18.0) ;{ }^{13} \mathrm{C}$ NMR (100 MHz) $24.7\left(\mathrm{CH}_{3}\right), 30.1(\mathrm{C}-3), 31.9(\mathrm{C}-4), 47.1(\mathrm{C}-1), 51.8$ (C-2), $83.1\left(C\left(\mathrm{CH}_{3}\right)_{2}\right), 152.7(\mathrm{C}-5, \mathrm{C}-6)$; MS (CI+) $\mathrm{m} / z: 242.2\left(\mathrm{MNH}_{4}^{+}, 100 \%\right), 225.2(6 \%)$; HRMS $m / z: \mathrm{MNH}_{4}{ }^{+}$found 242.1935, $\mathrm{C}_{12} \mathrm{H}_{25} \mathrm{BNO}_{3}$ requires 242.1927. 


\section{3,3-Diallylbicyclo[3.1.0]hexan-2-ol 5g}

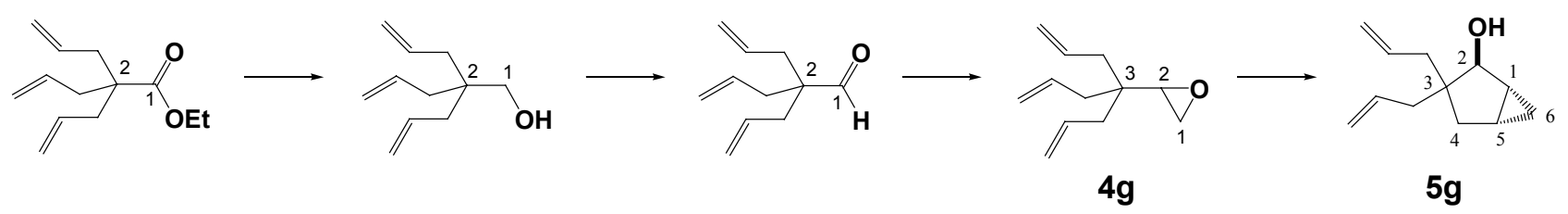

\section{3,3-Diallyl-1,2-epoxy-5-hexene $4 \mathrm{~g}$}

$n$-BuLi (1.6 $\mathrm{M}$ in hexane, $0.81 \mathrm{~mL}, 1.3 \mathrm{mmol}, 1.1$ equiv.) was added to a stirred solution of diisopropylamine $(0.18 \mathrm{~mL}, 1.31 \mathrm{mmol}, 1.1$ equiv. $)$ in $\mathrm{THF}(6 \mathrm{~mL})$ at $-78{ }^{\circ} \mathrm{C}$. The LDA solution formed was warmed to $\mathrm{rt}$ over $15 \mathrm{~min}$, cooled to $-78{ }^{\circ} \mathrm{C}$ and then treated slowly with ethyl 2allylpent-4-enoate $^{[9]}(200 \mathrm{mg}, 1.19 \mathrm{mmol})$. After $1 \mathrm{~h}$, the mixture was treated with allyl bromide ( $0.11 \mathrm{~mL}, 1.31 \mathrm{mmol}, 1.1$ equiv.), then stirred for $30 \mathrm{~min}$ and then left at at $\mathrm{rt}$ for $20 \mathrm{~h}$. The reaction mixture was poured into sat. aq. $\mathrm{NH}_{4} \mathrm{Cl}(30 \mathrm{~mL})$ and the aqueous layer extracted with $\mathrm{Et}_{2} \mathrm{O}(2 \times 30$ $\mathrm{mL})$. The combined organic layers were dried $\left(\mathrm{MgSO}_{4}\right)$, filtered and concentrated to give the crude product. Purification by column chromatography $\left(5 \% \mathrm{Et}_{2} \mathrm{O}\right.$ in petrol) gave ethyl 2,2-diallylpent-4enoate (234 mg, $1.12 \mathrm{mmol}, 94 \%)$ as a colorless oil, $R_{\mathrm{f}}=0.4\left(5 \% \mathrm{Et}_{2} \mathrm{O}\right.$ in petrol); IR (film) $3076 \mathrm{~m}$ $(\mathrm{C}=\mathrm{C}), 2980 \mathrm{~m}, 2932 \mathrm{~m}(\mathrm{C}-\mathrm{H}), 1725 \mathrm{~s}(\mathrm{C}=\mathrm{O}), 1638 \mathrm{~m}(\mathrm{C}=\mathrm{C}), 1441 \mathrm{~m}, 1369 \mathrm{w}, 1340 \mathrm{w}, 1297 \mathrm{w}, 1278 \mathrm{w}$, 1205s, 1148m, 1037m; ${ }^{1} \mathrm{H}$ NMR (400 MHz) $1.26\left(\mathrm{t}, 3 \mathrm{H}, \mathrm{OCH}_{2} \mathrm{CH}_{3}, J=7.2\right), 2.34(\mathrm{~d}, 6 \mathrm{H}, 3 \times$ $\left.\mathrm{CH}_{2} \mathrm{CH}=\mathrm{CH}_{2}, J=7.6\right), 4.15\left(\mathrm{q}, 2 \mathrm{H}, \mathrm{OCH}_{2} \mathrm{CH}_{3}, J=7.2\right), 5.07-5.10\left(\mathrm{~m}, 6 \mathrm{H}, 3 \times \mathrm{CH}_{2} \mathrm{CH}=\mathrm{CH}_{2}\right), 5.67$ - $5.78\left(\mathrm{~m}, 3 \mathrm{H}, 3 \times \mathrm{CH}_{2} \mathrm{CH}=\mathrm{CH}_{2}\right) ;{ }^{13} \mathrm{C} \mathrm{NMR}(100 \mathrm{MHz}) 14.3\left(\mathrm{OCH}_{2} \mathrm{CH}_{3}\right), 38.9\left(\mathrm{CH}_{2} \mathrm{CH}=\mathrm{CH}_{2}\right), 49.1$ (C-2), $60.4\left(\mathrm{OCH}_{2} \mathrm{CH}_{3}\right), 118.3\left(\mathrm{CH}_{2} \mathrm{CH}=\mathrm{CH}_{2}\right), 133.5\left(\mathrm{CH}_{2} \mathrm{CH}=\mathrm{CH}_{2}\right), 175.4(\mathrm{C}=\mathrm{O})$.

DIBALH (1.0 M in hexane, $27.1 \mathrm{~mL}, 27.1 \mathrm{mmol}, 2.2$ equiv.) was added slowly to a stirred solution of ethyl 2,2-diallylpent-4-enoate $(2.56 \mathrm{~g}, 12.3 \mathrm{mmol})$ in $\mathrm{CH}_{2} \mathrm{Cl}_{2}(55 \mathrm{~mL})$ at $-78{ }^{\circ} \mathrm{C}$. After $3 \mathrm{~h}$, the mixture was treated slowly with $\mathrm{MeOH}(0.5 \mathrm{~mL})$ and then warmed to rt. The reaction mixture was washed successively with water $(150 \mathrm{~mL})$ and aq. $\mathrm{HCl}(2 \mathrm{M}, 50 \mathrm{~mL})$, and the aqueous layers were extracted with $\mathrm{CH}_{2} \mathrm{Cl}_{2}(100 \mathrm{~mL})$. The combined organic layers were dried $\left(\mathrm{MgSO}_{4}\right)$, filtered and concentrated to give 2,2-diallylpent-4-en-1-ol (1.934 g, $11.63 \mathrm{mmol}, 95 \%)$ as a pale yellow oil, which was sufficiently pure to be used without further purification, $R_{\mathrm{f}}=0.2\left(30 \% \mathrm{Et}_{2} \mathrm{O}\right.$ in petrol); IR (film) 3399bs (O-H), 3075m (C=C), 3005w, 2978m, 2924m (C-H), 1639m (C=C), 1444m, 1415w, 
1262m, 1213w, 1045s, 1017s; ${ }^{1} \mathrm{H}$ NMR (400 MHz) 2.07 (d, 6H, $\left.3 \times \mathrm{CH}_{2} \mathrm{CH}=\mathrm{CH}_{2}, J=7.2\right), 3.42(\mathrm{~s}$, $\left.2 \mathrm{H}, \mathrm{CH}_{2} \mathrm{OH}\right), 5.09-5.12\left(\mathrm{~m}, 6 \mathrm{H}, 3 \times \mathrm{CH}_{2} \mathrm{CH}=\mathrm{CH}_{2}\right), 5.82-5.93\left(\mathrm{~m}, 3 \mathrm{H}, 3 \times \mathrm{CH}_{2} \mathrm{CH}=\mathrm{CH}_{2}\right) ;{ }^{13} \mathrm{C}$ NMR (100 MHz) $38.8\left(\mathrm{CH}_{2}=\mathrm{CHCH}_{2}\right), 41.2(\mathrm{C}-2), 67.5(\mathrm{C}-1), 117.8\left(\mathrm{CH}_{2} \mathrm{CH}=\mathrm{CH}_{2}\right), 134.5$ $\left(\mathrm{CH}_{2} \mathrm{CH}=\mathrm{CH}_{2}\right) ; \mathrm{MS}(\mathrm{CI}+) \mathrm{m} / \mathrm{z}: 184.2\left(\mathrm{MNH}_{4}^{+}, 100 \%\right), 167.1\left(\mathrm{MH}^{+}, 70 \%\right), 151.1(10 \%), 137.1$ (50\%), 124.1 (20\%), 107.1 (18\%); HRMS m/z: $\mathrm{MNH}_{4}^{+}$found 184.1693, $\mathrm{C}_{11} \mathrm{H}_{22} \mathrm{NO}$ requires 184.1701.

DMSO (2.1 mL, $29.0 \mathrm{mmol}, 2.5$ equiv.) was added slowly to a stirred solution of oxalyl chloride (1.1 mL, $12.8 \mathrm{mmol}, 1.1$ equiv.) in $\mathrm{CH}_{2} \mathrm{Cl}_{2}(70 \mathrm{~mL})$ at $-78{ }^{\circ} \mathrm{C}$. The resulting mixture was stirred for $5 \mathrm{~min}$, treated slowly with a solution of 2,2-diallylpent-4-en-1-ol (1.93 g, $11.6 \mathrm{mmol})$ in $\mathrm{CH}_{2} \mathrm{Cl}_{2}(10$ $\mathrm{mL})$ and then stirred for a further $15 \mathrm{~min}$. The mixture was treated slowly with $\mathrm{Et}_{3} \mathrm{~N}(8.1 \mathrm{~mL}, 58.0$ mmol, 5.0 equiv.), stirred for $5 \mathrm{~min}$ and then warmed to $\mathrm{rt}$. After $3 \mathrm{~h}$, water $(100 \mathrm{~mL})$ was added to the reaction mixture, the layers were separated and the aqueous layer extracted with $\mathrm{CH}_{2} \mathrm{Cl}_{2}(2 \times 100$ $\mathrm{mL})$. The combined organic layers were dried $\left(\mathrm{MgSO}_{4}\right)$, filtered and concentrated to give the crude product. Purification by column chromatography $\left(10 \% \mathrm{Et}_{2} \mathrm{O}\right.$ in petrol) gave 2,2-diallylpent-4-enal $(1.86 \mathrm{~g}, 11.3 \mathrm{mmol}, 97 \%)$ as a colorless oil, $R_{\mathrm{f}}=0.6\left(10 \% \mathrm{Et}_{2} \mathrm{O}\right.$ in petrol); IR (film) $3076 \mathrm{~m}(\mathrm{C}=\mathrm{C})$, 2980m, 2922m (C-H), 2836w, 2720w, 1725s (C=O), 1638m (C=C), 1441m, 1417w; ${ }^{1} \mathrm{H}$ NMR (400 $\mathrm{MHz}) 2.28\left(\mathrm{dt}, 6 \mathrm{H}, 3 \times \mathrm{CH}_{2} \mathrm{CH}=\mathrm{CH}_{2}, J=1.2,7.6\right), 5.07-5.12\left(\mathrm{~m}, 6 \mathrm{H}, 3 \times \mathrm{CH}_{2} \mathrm{CH}=\mathrm{CH}_{2}\right), 5.65-$ $5.75\left(\mathrm{~m}, 3 \mathrm{H}, 3 \times \mathrm{CH}_{2} \mathrm{CH}=\mathrm{CH}_{2}\right), 9.52(\mathrm{~s}, 1 \mathrm{H}, \mathrm{CHO}) ;{ }^{13} \mathrm{C} \mathrm{NMR}(100 \mathrm{MHz}) 36.7\left(\mathrm{CH}_{2} \mathrm{CH}=\mathrm{CH}_{2}\right), 51.8$ (C-2), $118.9\left(\mathrm{CH}_{2} \mathrm{CH}=\mathrm{CH}_{2}\right), 132.5\left(\mathrm{CH}_{2} \mathrm{CH}=\mathrm{CH}_{2}\right), 205.6(\mathrm{C}=\mathrm{O}) ; \mathrm{MS}(\mathrm{CI}+) \mathrm{m} / z$ : $182.2\left(\mathrm{MNH}_{4}^{+}\right.$, 100\%), $165.1\left(\mathrm{MH}^{+}, 15 \%\right), 135.1(17 \%), 123.1(20 \%), 109.1(7 \%) ;$ HRMS $\mathrm{m} / z: \mathrm{MNH}_{4}^{+}$found 182.1545, $\mathrm{C}_{11} \mathrm{H}_{20} \mathrm{NO}$ requires 182.1545 .

$n$-BuLi (1.6 M in hexane, $2.5 \mathrm{~mL}, 4.0 \mathrm{mmol}, 1.1$ equiv.) was added dropwise ( $1 \mathrm{drop} / 2 \mathrm{~s})$ to a stirred solution of 2,2-diallylpent-4-enal $(600 \mathrm{mg}, 3.65 \mathrm{mmol})$ and dibromomethane $(0.38 \mathrm{~mL}, 5.48$ mmol, 1.5 equiv. $)$ in THF $(15 \mathrm{~mL})$ at $-78{ }^{\circ} \mathrm{C} .^{[6]}$ The resulting mixture was warmed to $\mathrm{rt}$ and stirred for a further $16 \mathrm{~h}$. The reaction mixture was washed with sat. aq. $\mathrm{NH}_{4} \mathrm{Cl}(50 \mathrm{~mL})$, the layers were separarted and the aqueous layer extracted with $\mathrm{Et}_{2} \mathrm{O}(2 \times 40 \mathrm{~mL})$. The combined organic layers 
were dried $\left(\mathrm{MgSO}_{4}\right)$, filtered and concentrated to give the crude product. Purification by column chromatography $\left(10 \% \mathrm{Et}_{2} \mathrm{O}\right)$ gave epoxide $4 \mathrm{~g}(437 \mathrm{mg}, 2.45 \mathrm{mmol}, 67 \%)$ as a colorless oil, $R_{\mathrm{f}}=0.5$ (10\% $\mathrm{Et}_{2} \mathrm{O}$ in petrol); IR (film) 3076m (epoxide), 3006w, 2979m, 2924s (C-H), 1640m (C=C), 1485w, 1445m, 1274w; ${ }^{1} \mathrm{H}$ NMR (400 MHz) $2.01-2.14\left(\mathrm{~m}, 6 \mathrm{H}, 3 \times \mathrm{CH}_{2} \mathrm{CH}=\mathrm{CH}_{2}\right), 2.64-2.69(\mathrm{~m}$, $2 \mathrm{H}, 2 \times \mathrm{H}-1), 2.88(\mathrm{dd}, 1 \mathrm{H}, \mathrm{H}-2, J=3.2,4.0), 5.06-5.10\left(\mathrm{~m}, 6 \mathrm{H}, 3 \times \mathrm{CH}_{2} \mathrm{CH}=\mathrm{CH}_{2}\right), 5.80-5.91(\mathrm{~m}$, $\left.3 \mathrm{H}, 3 \times \mathrm{CH}_{2} \mathrm{CH}=\mathrm{CH}_{2}\right) ;{ }^{13} \mathrm{C} \mathrm{NMR}(100 \mathrm{MHz}) 38.6(\mathrm{C}-3), 38.8\left(\mathrm{CH}_{2} \mathrm{CH}=\mathrm{CH}_{2}\right), 43.6(\mathrm{C}-1), 57.9(\mathrm{C}-$ 2), $118.0\left(\mathrm{CH}_{2} \mathrm{CH}=\mathrm{CH}_{2}\right), 134.0\left(\mathrm{CH}_{2} \mathrm{CH}=\mathrm{CH}_{2}\right) ; \mathrm{MS}(\mathrm{CI}+) \mathrm{m} / z: 196.2\left(\mathrm{MNH}_{4}^{+}, 75 \%\right), 179.1\left(\mathrm{MH}^{+}\right.$, 100\%), 161.1 (100\%), 137.1 (30\%), 119.1 (30\%), $107.1(10 \%) ; \mathrm{HRMS} \mathrm{m} / z: \mathrm{MNH}_{4}^{+}$found 196.1700, $\mathrm{C}_{12} \mathrm{H}_{22} \mathrm{NO}$ requires $196.1701, \mathrm{MH}^{+}$found $179.1432, \mathrm{C}_{12} \mathrm{H}_{19} \mathrm{O}$ requires 179.1436 .

\section{3,3-Diallylbicyclo[3.1.0]hexan-2-ol 5g}

Following the typical cyclopropanation procedure, 3,3-diallyl-1,2-epoxy-5-hexene $\mathbf{4 g}$ (178 $\mathrm{mg}, 1.00$ mmol) was treated with LTMP in $\mathrm{Et}_{2} \mathrm{O}$ for $8 \mathrm{~h}$. TLC analysis of the reaction mixture revealed the presence of the starting epoxide, $R_{\mathrm{f}}=0.8\left(50 \% \mathrm{Et}_{2} \mathrm{O}\right.$ in petrol $)$ and product, $R_{\mathrm{f}}=0.3\left(50 \% \mathrm{Et}_{2} \mathrm{O}\right.$ in petrol). The reaction mixture was cooled to $0{ }^{\circ} \mathrm{C}$ and treated with another batch of LTMP (1.5 equiv.) under the standard conditions. After a further $8 \mathrm{~h}$, TLC analysis showed complete consumption of starting material. Bicyclic alcohol 5g (149 mg, $0.84 \mathrm{mmol}, 84 \%)$ was subsequently isolated as a colorless oil following the standard purification procedures, $R_{\mathrm{f}}=0.3\left(50 \% \mathrm{Et}_{2} \mathrm{O}\right.$ in petrol); IR (film) 3390bs (O-H), 3074s (cyclopropane), 3030w, 2978s, 2919s, 2861s (C-H), 1638s $(\mathrm{C}=\mathrm{C}), 1440 \mathrm{~m}, 1328 \mathrm{w}, 1303 \mathrm{w}, 1244 \mathrm{w}, 1077 \mathrm{~m}, 1057 \mathrm{~m}, 1028 \mathrm{~s} ;{ }^{1} \mathrm{H}$ NMR $(400 \mathrm{MHz}) 0.24-0.27$ (m, $1 \mathrm{H}, \mathrm{H}-6_{\text {endo }}$ ), $0.78-0.83$ (m, 1H, H-6 exo $), 1.22$ (dd, $\left.1 \mathrm{H}, \mathrm{H}-4, J=2.0,14.0\right), 1.29-1.38$ (m, 2H, H-1, $\mathrm{H}-5), 1.76(\mathrm{~s}, \mathrm{OH}), 1.91(\mathrm{dd}, 1 \mathrm{H}, \mathrm{H}-4, J=6.0,14.0), 1.97-2.09\left(\mathrm{~m}, 2 \mathrm{H}, \mathrm{CH}_{2} \mathrm{CH}=\mathrm{CH}_{2}\right), 2.27(\mathrm{~d}, 2 \mathrm{H}$, $\left.\mathrm{CH}_{2} \mathrm{CH}=\mathrm{CH}_{2}, J=7.2\right), 3.75(\mathrm{~d}, 1 \mathrm{H}, \mathrm{H}-2, J=4.8), 5.01-5.15\left(\mathrm{~m}, 4 \mathrm{H}, 2 \times \mathrm{CH}_{2} \mathrm{CH}=\mathrm{CH}\right)_{2}, 5.78-6.01$ $\left(\mathrm{m}, 2 \mathrm{H}, 2 \times \mathrm{CH}_{2} \mathrm{CH}=\mathrm{CH}_{2}\right) ;{ }^{13} \mathrm{C} \mathrm{NMR}(100 \mathrm{MHz}) 17.2(\mathrm{C}-5, \mathrm{C}-6), 26.0(\mathrm{C}-1), 38.1\left(\mathrm{CH}_{2} \mathrm{CH}=\mathrm{CH}_{2}\right)$, $38.2(\mathrm{C}-4), 42.7\left(\mathrm{CH}_{2} \mathrm{CH}=\mathrm{CH}_{2}\right), 55.0(\mathrm{C}-3), 82.2(\mathrm{C}-2), 117.1\left(\mathrm{CH}_{2} \mathrm{CH}=\mathrm{CH}_{2}\right), 117.3\left(\mathrm{CH}_{2} \mathrm{CH}=\mathrm{CH}_{2}\right)$, $135.4\left(\mathrm{CH}_{2} \mathrm{CH}=\mathrm{CH}_{2}\right), 136.2\left(\mathrm{CH}_{2} \mathrm{CH}=\mathrm{CH}_{2}\right) ; \mathrm{MS}(\mathrm{CI}+) \mathrm{m} / z: 196.2\left(\mathrm{MNH}_{4}^{+}, 20 \%\right), 179.1\left(\mathrm{MH}^{+}\right.$, 35\%), 161.1 (100\%), $137.1(10 \%), 119.1(20 \%), 108.1$ (4\%); HRMS $m / z: \mathrm{MNH}_{4}^{+}$found 196.1703, $\mathrm{C}_{12} \mathrm{H}_{22} \mathrm{NO}$ requires 196.1701 . 


\section{3-Allylbicyclo[3.1.0]hexan-2-ol 5h}

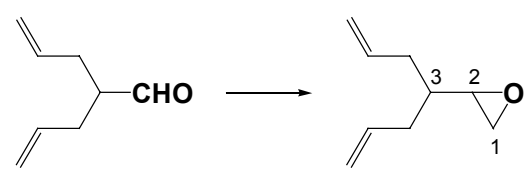

$4 h$

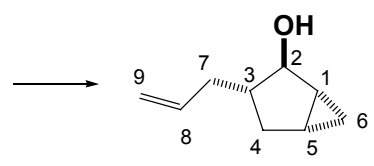

$5 \mathrm{~h}$

2-(1-Allylbut-3-enyl)oxirane $4 \boldsymbol{h}$

$n$-BuLi (1.6 M in hexane, $2.2 \mathrm{~mL}, 3.5 \mathrm{mmol}, 1.1$ equiv.) was added dropwise ( $1 \mathrm{drop} / 3 \mathrm{~s})$ to a stirred solution of 2-allylpent-4-enal ${ }^{[10]}(400 \mathrm{mg}, 3.22 \mathrm{mmol})$ and dibromomethane $(0.27 \mathrm{~mL}, 3.86$ mmol, 1.2 equiv.) in THF $(10 \mathrm{~mL})$ at $-78{ }^{\circ} \mathrm{C} .^{[6]}$ The resulting mixture was warmed to $\mathrm{rt}$ and stirred for $16 \mathrm{~h}$. The reaction mixture was washed with sat. aq. $\mathrm{NH}_{4} \mathrm{Cl}(50 \mathrm{~mL})$, the layers were separated and the aqueous layer extracted with $\mathrm{Et}_{2} \mathrm{O}(2 \times 40 \mathrm{~mL})$. The combined organic layers were dried $\left(\mathrm{MgSO}_{4}\right)$, filtered and concentrated to give the crude product. Purification by column chromatography $\left(10 \% \mathrm{Et}_{2} \mathrm{O}\right)$ gave epoxide $4 \mathrm{~h}(289 \mathrm{mg}, 2.09 \mathrm{mmol}, 65 \%)$ as a colorless oil, $R_{\mathrm{f}}=0.5$ (10\% $\mathrm{Et}_{2} \mathrm{O}$ in petrol); IR (film) 3078m (C=C), 2997m, 2979s, 2920s (C-H), 1641s (C=C), 1442m, 1417w, 1261w; ${ }^{1} \mathrm{H}$ NMR (400 MHz) $1.30-1.38$ (m, 1H, H-3), $2.06-2.34$ (m, 4H, $2 \times$ $\left.\mathrm{CH}_{2} \mathrm{CH}=\mathrm{CH}_{2}\right), 2.52-2.54(\mathrm{~m}, 1 \mathrm{H}, \mathrm{H}-1), 2.76-2.80\left(\mathrm{~m}, 2 \mathrm{H}, \mathrm{H}-1^{\prime}, \mathrm{H}-2\right), 5.02-5.12(\mathrm{~m}, 4 \mathrm{H}, 2 \times$ $\left.\mathrm{CH}_{2} \mathrm{CH}=\mathrm{CH}_{2}\right), 5.73-5.90\left(\mathrm{~m}, 2 \mathrm{H}, 2 \times \mathrm{CH}_{2} \mathrm{CH}=\mathrm{CH}_{2}\right) ;{ }^{13} \mathrm{C}$ NMR $(100 \mathrm{MHz})$ 35.1, $36.3(2 \times$ $\left.\mathrm{CH}_{2} \mathrm{CH}=\mathrm{CH}_{2}\right), 41.3(\mathrm{C}-3), 46.9(\mathrm{C}-1), 55.2(\mathrm{C}-2), 116.6,116.7\left(\mathrm{CH}_{2} \mathrm{CH}=\mathrm{CH}_{2}\right), 135.9,136.0$ $\left(\mathrm{CH}_{2} \mathrm{CH}=\mathrm{CH}_{2}\right) ; \mathrm{MS}(\mathrm{CI}+) \mathrm{m} / z: 156.1\left(\mathrm{MNH}_{4}^{+}, 100 \%\right), 139.1\left(\mathrm{MH}^{+}, 53 \%\right), 121.1(74 \%), 107.1$ (17\%); HRMS $m / z: \mathrm{MNH}_{4}{ }^{+}$found 156.1391, $\mathrm{C}_{9} \mathrm{H}_{18} \mathrm{NO}$ requires 156.1388.

\section{3-Allylbicyclo[3.1.0]hexan-2-ol $5 \boldsymbol{h}$}

Following the typical cyclopropanation procedure, 2-(1-allylbut-3-enyl)oxirane $\mathbf{4 h}$ (138 $\mathrm{mg}, 1.00$ mmol) was treated with LTMP for $10 \mathrm{~h}$ to give 3-allylbicyclo[3.1.0]hexan-2-ol $\mathbf{5 h}$ (96 $\mathrm{mg}, 0.69$ mmol, 69\%) as a colorless oil, $R_{\mathrm{f}}=0.3\left(50 \% \mathrm{Et}_{2} \mathrm{O}\right.$ in petrol); IR (film) $3330 \mathrm{bs}(\mathrm{O}-\mathrm{H}), 3070 \mathrm{~m}$ (cyclopropane), 2926s, 2868s $(\mathrm{C}-\mathrm{H}), 1636 \mathrm{~m}(\mathrm{C}=\mathrm{C}), 1438 \mathrm{~m}, 1414 \mathrm{~m}, 1356 \mathrm{~m}, 1342 \mathrm{~m}, 1265 \mathrm{w}, 1212 \mathrm{w}$, 1029s; ${ }^{1} \mathrm{H}$ NMR (400 MHz) $0.07-0.10$ (m, 1H, H-6 endo), 0.56 - 0.62 (m, 1H, H- $6_{\text {exo }}$ ), 1.34 - 1.41 (m, 2H, H-1, H-4), 1.43 - 1.50 (m, 1H, H-5), 1.70 (s, 1H, OH), 1.88 - 1.96 (m, 1H, H-7), 2.04 - 2.18 (m, 2H, H-3, H-7'), 2.25 - 2.32 (m, 1H, H-4'), 3.95 (d, 1H, H-2, J=2.8), 4.97 - 5.02 (m, 2H, $2 \times$ H-9), 
5.69 - 5.79 (m, 1H, H-8); ${ }^{13} \mathrm{C}$ NMR (100 MHz) 10.6 (C-6), 18.5 (C-5), 26.4 (C-1), 32.0 (C-4), 40.6 (C-7), 50.0 (C-3), 80.2 (C-2), 115.5 (C-9), 137.5 (C-8); MS (CI+) m/z: $156.1\left(\mathrm{MNH}_{4}^{+}, 10 \%\right), 139.1$ $\left(\mathrm{MH}^{+}, 20 \%\right), 121.1(67 \%), 105.1(4 \%)$; HRMS $m / z: \mathrm{MNH}_{4}{ }^{+}$found $156.1384, \mathrm{C}_{9} \mathrm{H}_{18} \mathrm{NO}$ requires 156.1388.

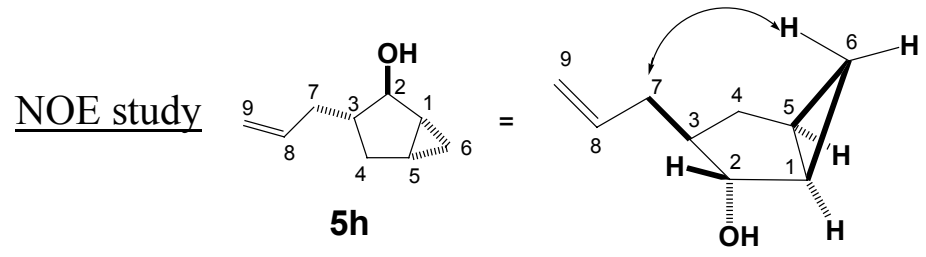

NOE study: irradiation of $\mathrm{H}-6_{\text {endo }}$ and $\mathrm{H}-7$ s showed reciprocal signal enhancement, but no signal enhancement was observed between $\mathrm{H}-6_{\text {endo }}$ and $\mathrm{H}-3$.

\section{3-Allyl-3-isopropylbicyclo[3.1.0]hexan-2-ol 5i}
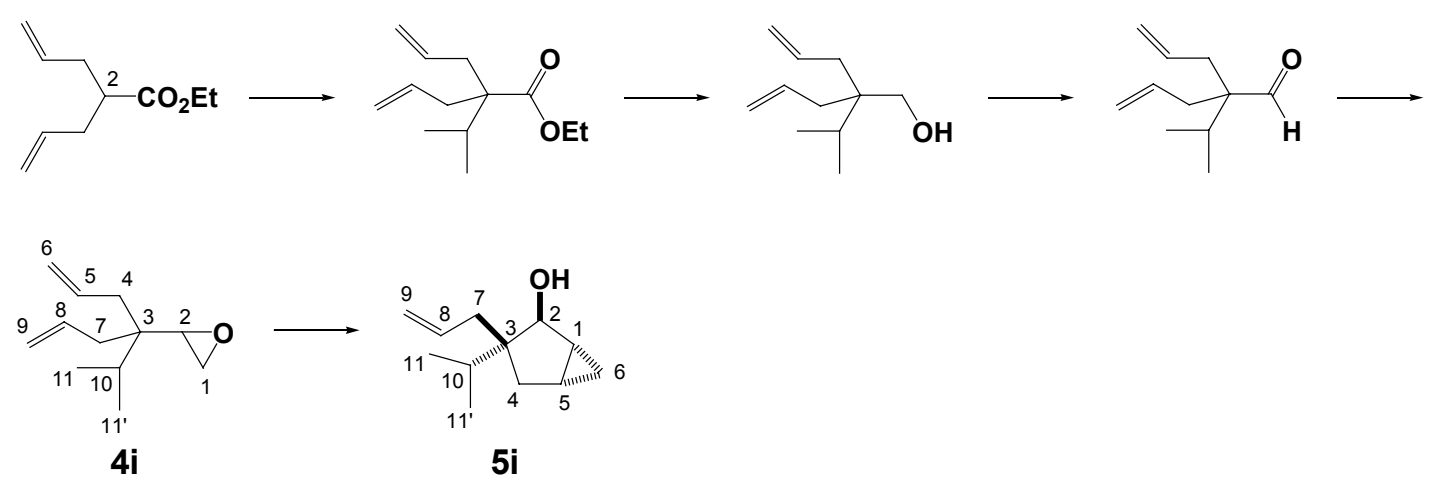

2-(1-Allyl-1-isopropylbut-3-enyl)oxirane $4 \boldsymbol{i}$

$n$-BuLi (1.6 $\mathrm{M}$ in hexane, $0.8 \mathrm{~mL}, 1.3 \mathrm{mmol}, 1.1$ equiv.) was added to a stirred solution of diisopropylamine $(0.18 \mathrm{~mL}, 1.31 \mathrm{mmol}, 1.1$ equiv. $)$ in THF $(6 \mathrm{~mL})$ at $-78{ }^{\circ} \mathrm{C}$. The LDA solution formed was warmed to $\mathrm{rt}$ over $15 \mathrm{~min}$, then cooled to $-78{ }^{\circ} \mathrm{C}$. Ethyl 2-allylpent-4-enoate ${ }^{[9]}(200 \mathrm{mg}$, $1.19 \mathrm{mmol}$ ) was added slowly to the mixture. The resulting mixture was stirred for a $1 \mathrm{~h}$ at the same temperature and treated with isopropyliodide $(3.57 \mathrm{mmol}, 608 \mathrm{mg}, 0.35 \mathrm{~mL}, 3.0$ equiv.). After 30 min, the mixture was warmed to $\mathrm{rt}$ and then stirred for $20 \mathrm{~h}$. The reaction mixture was poured into sat. aq. $\mathrm{NH}_{4} \mathrm{Cl}(40 \mathrm{~mL})$, the layers were separated and the aqueous layer extracted with $\mathrm{Et}_{2} \mathrm{O}(50$ $\mathrm{mL})$. The combined organic layers were dried $\left(\mathrm{MgSO}_{4}\right)$, filtered and concentrated to give the crude product. Purification by column chromatography $\left(5 \% \mathrm{Et}_{2} \mathrm{O}\right.$ in petrol) gave ethyl 2-allyl-2isopropylpent-4-enoate (197 $\mathrm{mg}, 0.94 \mathrm{mmol}, 79 \%)$ as a colorless oil, $R_{\mathrm{f}}=0.4\left(5 \% \mathrm{Et}_{2} \mathrm{O}\right.$ in petrol); IR (film) 3076m (C=C), 2970s $(\mathrm{CH}), 1725 \mathrm{~s}(\mathrm{C}=\mathrm{O}), 1638 \mathrm{~m}(\mathrm{C}=\mathrm{C}), 1446 \mathrm{~m}, 1388 \mathrm{w}, 1369 \mathrm{w}, 1210 \mathrm{~s}$, 
1143s, 1032m; ${ }^{1} \mathrm{H}$ NMR (400 MHz) $0.93\left(\mathrm{~d}, 6 \mathrm{H}, \mathrm{CH}\left(\mathrm{CH}_{3}\right)_{2}, J=6.8\right), 1.27$ (t, 3H, $\mathrm{OCH}_{2} \mathrm{CH}_{3}, J=$ 7.2), 1.93 (sept, $\left.1 \mathrm{H}, \mathrm{CH}\left(\mathrm{CH}_{3}\right)_{2}, J=7.2\right), 2.32\left(\mathrm{dd}, 2 \mathrm{H}, \mathrm{CH}_{2} \mathrm{CH}=\mathrm{CH}_{2}, J=8.0,14.4\right), 2.50$ (dd, $2 \mathrm{H}$, $\left.\mathrm{CH}_{2} \mathrm{CH}=\mathrm{CH}_{2}, J=6.8,14.4\right), 4.15\left(\mathrm{q}, 2 \mathrm{H}, \mathrm{OCH}_{2} \mathrm{CH}_{3}, J=7.6\right), 5.02-5.10(\mathrm{~m}, 4 \mathrm{H}, 2 \times$ $\left.\mathrm{CH}_{2} \mathrm{CH}=\mathrm{CH}_{2}\right), 5.74-5.85\left(\mathrm{~m}, 2 \mathrm{H}, 2 \times \mathrm{CH}_{2} \mathrm{CH}=\mathrm{CH}_{2}\right) ;{ }^{13} \mathrm{C} \mathrm{NMR}(100 \mathrm{MHz}) 14.3\left(\mathrm{OCH}_{2} \mathrm{CH}_{3}\right), 17.9$ $\left(\mathrm{CH}\left(\mathrm{CH}_{3}\right)_{2}\right), \quad 33.1 \quad\left(\mathrm{CH}\left(\mathrm{CH}_{3}\right)_{2}\right), \quad 37.1 \quad\left(\mathrm{CH}_{2} \mathrm{CH}=\mathrm{CH}_{2}\right), \quad 52.0 \quad(\mathrm{C}-2), \quad 60.1 \quad\left(\mathrm{OCH}_{2} \mathrm{CH}_{3}\right), \quad 117.6$ $\left(\mathrm{CH}_{2} \mathrm{CH}=\mathrm{CH}_{2}\right), 134.9\left(\mathrm{CH}_{2} \mathrm{CH}=\mathrm{CH}_{2}\right), 175.2(\mathrm{C}-1) ; \mathrm{MS}(\mathrm{CI}+) m / z: 228.2\left(\mathrm{MNH}_{4}{ }^{+}, 5 \%\right), 211.2$ $\left(\mathrm{MH}^{+}, 100 \%\right), 167.1$ (5\%), 148.1 (5\%), 137.1 (2\%); HRMS $m / z: \mathrm{MNH}_{4}{ }^{+}$found 228.1967, $\mathrm{C}_{13} \mathrm{H}_{26} \mathrm{NO}_{2}$ requires 228.1964 .

DIBALH (1.0 M in hexane, $32.3 \mathrm{~mL}, 32.3 \mathrm{mmol}, 2.1$ equiv.) was added slowly to a stirred solution of ethyl 2-allyl-2-isopropylpent-4-enoate $(3.23 \mathrm{~g}, 15.4 \mathrm{mmol})$ in $\mathrm{CH}_{2} \mathrm{Cl}_{2}(60 \mathrm{~mL})$ at $-78{ }^{\circ} \mathrm{C}$. After 3 $\mathrm{h}$, the mixture was treated slowly with $\mathrm{MeOH}(0.5 \mathrm{~mL})$ and then warmed to rt. The reaction mixture was washed with water $(150 \mathrm{~mL})$, followed by aq. $\mathrm{HCl}(2 \mathrm{M}, 50 \mathrm{~mL})$. The aqueous layers were extracted with $\mathrm{CH}_{2} \mathrm{Cl}_{2}(100 \mathrm{~mL})$, and then the combined organic layers were dried $\left(\mathrm{MgSO}_{4}\right)$, filtered and concentrated to give the crude product. Purification by column chromatography $\left(10 \% \mathrm{Et}_{2} \mathrm{O}\right.$ in petrol) gave 2-allyl-2-isopropylpent-4-en-1-ol $(2.54 \mathrm{~g}, 15.1 \mathrm{mmol}, 98 \%)$ as a colorless oil, $R_{\mathrm{f}}=0.3$ $\left(10 \% \mathrm{Et}_{2} \mathrm{O}\right.$ in petrol); IR (film) 3433bs $(\mathrm{O}-\mathrm{H}), 3078 \mathrm{~m}(\mathrm{C}=\mathrm{C}), 2969 \mathrm{~s}, 2889 \mathrm{~s}(\mathrm{C}-\mathrm{H}), 1630 \mathrm{~m}(\mathrm{C}=\mathrm{C})$, 1467m, 1442m, 1383w, 1363w, 1038m; ${ }^{1} \mathrm{H}$ NMR (400 MHz) 0.93 (d, 6H, $\left.\mathrm{CH}\left(\mathrm{CH}_{3}\right)_{2}, J=6.8\right), 1.46$ (bs, 1H, OH), 1.82 (sept, 1H, $\left.\mathrm{CH}\left(\mathrm{CH}_{3}\right)_{2}, J=6.8\right), 2.13\left(\mathrm{~d}, 4 \mathrm{H}, 2 \times \mathrm{CH}_{2} \mathrm{CH}=\mathrm{CH}_{2}, J=7.6\right), 3.55(\mathrm{~d}$, $2 \mathrm{H}, 2 \times \mathrm{H}-1, J=3.6), 5.04-5.14\left(\mathrm{~m}, 4 \mathrm{H}, 2 \times \mathrm{CH}_{2} \mathrm{CH}=\mathrm{CH}_{2}\right), 5.89-6.00\left(\mathrm{~m}, 2 \mathrm{H}, 2 \times \mathrm{CH}_{2} \mathrm{CH}=\mathrm{CH}_{2}\right)$; ${ }^{13} \mathrm{C}$ NMR $(100 \mathrm{MHz}) 17.3\left(\mathrm{CH}\left(\mathrm{CH}_{3}\right)_{2}\right), 31.0\left(\mathrm{CH}\left(\mathrm{CH}_{3}\right)_{2}\right), 37.8\left(\mathrm{CH}_{2} \mathrm{CH}=\mathrm{CH}_{2}\right), 43.0(\mathrm{C}-2), 67.8(\mathrm{C}-$ 1), $117.2\left(\mathrm{CH}_{2} \mathrm{CH}=\mathrm{CH}_{2}\right), 135.9\left(\mathrm{CH}_{2} \mathrm{CH}=\mathrm{CH}_{2}\right) ; \mathrm{MS}(\mathrm{CI}+) \mathrm{m} / z: 186.2\left(\mathrm{MNH}_{4}^{+}, 100 \%\right), 169.2$ $\left(\mathrm{MH}^{+}, 93 \%\right), 151.1(15 \%), 137.1(20 \%), 125.1(15 \%), 109.1(30 \%)$; HRMS $m / z: \mathrm{MNH}_{4}^{+}$found 186.1862, $\mathrm{C}_{11} \mathrm{H}_{24} \mathrm{NO}$ requires $186.1858, \mathrm{MH}^{+}$found $169.1586, \mathrm{C}_{11} \mathrm{H}_{21} \mathrm{O}$ requires 169.1592.

DMSO (2.7 mL, $37.8 \mathrm{mmol}, 2.5$ equiv.) was added slowly to a stirred solution of oxalyl chloride (1.4 mL, $16.6 \mathrm{mmol}, 1.1$ equiv.) in $\mathrm{CH}_{2} \mathrm{Cl}_{2}(85 \mathrm{~mL})$ at $-78{ }^{\circ} \mathrm{C}$. After $5 \mathrm{~min}$, the mixture was treated slowly with a solution of 2-allyl-2-isopropylpent-4-en-1-ol (2.54 g, $15.1 \mathrm{mmol})$ in $\mathrm{CH}_{2} \mathrm{Cl}_{2}(15 \mathrm{~mL})$, 
stirred for $15 \mathrm{~min}$ and then treated slowly with $\mathrm{Et}_{3} \mathrm{~N}$ (10.5 mL, $75.5 \mathrm{mmol}, 5.0$ equiv.). The resulting mixture was stirred at the same temperature for $5 \mathrm{~min}$, warmed to $\mathrm{rt}$ and stirred for a further $3 \mathrm{~h}$. The reaction mixture was poured into water $(100 \mathrm{~mL})$, the layers were separated, and the aqueous layer extracted with $\mathrm{CH}_{2} \mathrm{Cl}_{2}(2 \times 100 \mathrm{~mL})$. The combined organic layers were dried $\left(\mathrm{MgSO}_{4}\right)$, filtered and concentrated to give the crude product. Purification by column chromatography (10\% $\mathrm{Et}_{2} \mathrm{O}$ in petrol) gave 2-allyl-2-isopropylpent-4-enal (2.29 g, $\left.13.8 \mathrm{mmol}, 91 \%\right)$ as a colorless oil, $R_{\mathrm{f}}=0.6\left(10 \% \mathrm{Et}_{2} \mathrm{O}\right.$ in petrol); IR (film) $3076 \mathrm{~m}(\mathrm{C}=\mathrm{C}), 2970 \mathrm{~s}(\mathrm{C}-\mathrm{H}), 2720 \mathrm{w}, 1725 \mathrm{~s}$ $(\mathrm{C}=\mathrm{O}), 1638 \mathrm{~m}(\mathrm{C}=\mathrm{C}), 1465 \mathrm{w}, 1441 \mathrm{w}, 1174 \mathrm{w} ;{ }^{1} \mathrm{H}$ NMR $(400 \mathrm{MHz}) 0.97\left(\mathrm{~d}, 6 \mathrm{H}, \mathrm{CH}\left(\mathrm{CH}_{3}\right)_{2}, J=7.2\right)$ 1.98 (sept, 1H, $\left.\mathrm{CH}\left(\mathrm{CH}_{3}\right)_{2}, J=7.2\right), 2.26-2.44\left(\mathrm{~m}, 4 \mathrm{H}, 2 \times \mathrm{CH}_{2} \mathrm{CH}=\mathrm{CH}_{2}\right), 5.07-5.13(\mathrm{~m}, 4 \mathrm{H}, 2 \times$ $\left.\mathrm{CH}_{2} \mathrm{CH}=\mathrm{CH}_{2}\right), 5.70-5.80\left(\mathrm{~m}, 2 \mathrm{H}, 2 \times \mathrm{CH}_{2} \mathrm{CH}=\mathrm{CH}_{2}\right) ;{ }^{13} \mathrm{C} \mathrm{NMR}(100 \mathrm{MHz}) 17.5\left(\mathrm{CH}\left(\mathrm{CH}_{3}\right)_{2}\right), 31.2$ $\left(\mathrm{CH}\left(\mathrm{CH}_{3}\right)_{2}\right), 34.8\left(2 \times \mathrm{CH}_{2} \mathrm{CH}=\mathrm{CH}_{2}\right), 53.9(\mathrm{C}-2), 118.3\left(2 \times \mathrm{CH}_{2} \mathrm{CH}=\mathrm{CH}_{2}\right), 133.6\left(\mathrm{CH}_{2} \mathrm{CH}=\mathrm{CH}_{2}\right)$, 206.8 (C-1); MS (CI+) m/z: $184.2\left(\mathrm{MNH}_{4}{ }^{+} 100 \%\right), 167.1\left(\mathrm{MH}^{+}, 30 \%\right), 151.1(12 \%), 137.1(17 \%)$, 124.1 (25\%), 109.1 (10\%); HRMS $m / z: \mathrm{MNH}_{4}{ }^{+}$found 184.1706, $\mathrm{C}_{11} \mathrm{H}_{22} \mathrm{NO}$ requires 184.1701.

$n$-BuLi (1.6 M in hexane, $2.5 \mathrm{~mL}, 4.0 \mathrm{mmol}, 1.1$ equiv.) was added dropwise ( $1 \mathrm{drop} / 3 \mathrm{~s})$ to a stirred solution of 2-allyl-2-isopropylpent-4-enal $(600 \mathrm{mg}, 3.61 \mathrm{mmol})$ and dibromomethane $(0.38$ $\mathrm{mL}, 5.42 \mathrm{mmol}, 1.5$ equiv.) in THF $(15 \mathrm{~mL})$ at $-78{ }^{\circ} \mathrm{C} .^{[6]}$ The resulting mixture was warmed to $\mathrm{rt}$ and stirred for a further $16 \mathrm{~h}$. The mixture was washed with sat. aq. $\mathrm{NH}_{4} \mathrm{Cl}(50 \mathrm{~mL})$, the layers were separated and the aqueous layer extracted with $\mathrm{Et}_{2} \mathrm{O}(2 \times 40 \mathrm{~mL})$. The combined organic layers were dried $\left(\mathrm{MgSO}_{4}\right)$, filtered and concentrated to give the crude product. Purification by column chromatography $\left(10 \% \mathrm{Et}_{2} \mathrm{O}\right)$ gave epoxide $4 \mathbf{i}(453 \mathrm{mg}, 2.51 \mathrm{mmol}, 70 \%)$ as a colorless oil, $R_{\mathrm{f}}=0.4$ $\left(10 \% \mathrm{Et}_{2} \mathrm{O}\right.$ in petrol); IR (film) 3076m $(\mathrm{CH}=\mathrm{C}), 2977 \mathrm{~s}, 2930 \mathrm{~s}(\mathrm{C}-\mathrm{H}), 1631 \mathrm{~m}(\mathrm{C}=\mathrm{C}), 1469 \mathrm{w}, 1440 \mathrm{w}$, 1386w, 1273w, 1175w; ${ }^{1} \mathrm{H}$ NMR (400 MHz) 0.98, 0.99 (2d, 6H, $\left.\mathrm{CH}\left(\mathrm{CH}_{3}\right)_{2}, J=6.8\right), 1.79$ (sept, 1H, $\left.\mathrm{CH}\left(\mathrm{CH}_{3}\right)_{2}, J=6.8\right), 1.96-2.18\left(\mathrm{~m}, 4 \mathrm{H}, 2 \times \mathrm{CH}_{2} \mathrm{CH}=\mathrm{CH}_{2}\right), 2.63(\mathrm{dd}, 1 \mathrm{H}, \mathrm{H}-1, J=4.4), 2.67(\mathrm{dd}, 1 \mathrm{H}$, $\left.\mathrm{H}-1^{\prime}, J=2.8,4.4\right), 2.90(\mathrm{dd}, 1-\mathrm{H}, \mathrm{H}-2, J=2.8,4.0), 5.02-5.12\left(\mathrm{~m}, 4 \mathrm{H}, 2 \times \mathrm{CH}_{2} \mathrm{CH}=\mathrm{CH}_{2}\right), 5.76-$ $5.94\left(\mathrm{~m}, 2 \mathrm{H}, 2 \times \mathrm{CH}_{2} \mathrm{CH}=\mathrm{CH}_{2}\right) ;{ }^{13} \mathrm{C} \mathrm{NMR}(100 \mathrm{MHz}) 17.3\left(\mathrm{CH}\left(\mathrm{CH}_{3}\right)_{2}\right), 17.6\left(\mathrm{CH}\left(\mathrm{CH}_{3}\right)_{2}\right), 33.1$ $\left(\mathrm{CH}\left(\mathrm{CH}_{3}\right)_{2}\right), 35.9\left(\mathrm{CH}_{2} \mathrm{CH}=\mathrm{CH}_{2}\right), 36.1\left(\mathrm{CH}_{2} \mathrm{CH}=\mathrm{CH}_{2}\right), 40.4(\mathrm{C}-3), 43.2(\mathrm{C}-1), 56.5(\mathrm{C}-2), 117.3$ $\left(\mathrm{CH}_{2} \mathrm{CH}=\mathrm{CH}_{2}\right), 117.4\left(\mathrm{CH}_{2} \mathrm{CH}=\mathrm{CH}_{2}\right), 134.7\left(\mathrm{CH}_{2} \mathrm{CH}=\mathrm{CH}_{2}\right), 134.8\left(\mathrm{CH}_{2} \mathrm{CH}=\mathrm{CH}_{2}\right) ; \mathrm{MS}(\mathrm{CI}+) m / z$ : 
$198.2\left(\mathrm{MNH}_{4}^{+} 30 \%\right), 181.2\left(\mathrm{MH}^{+}, 60 \%\right), 163.1(100 \%), 151.1(10 \%), 139.1(15 \%), 123.1(20 \%)$, 107.1 (15\%); HRMS $m / z: \mathrm{MNH}_{4}^{+}$found 198.1849, $\mathrm{C}_{12} \mathrm{H}_{24} \mathrm{NO}$ requires 198.1858.

\section{3-Allyl-3-isopropylbicyclo[3.1.0]hexan-2-ol 5i}

Following the typical cyclopropanation procedure, 2-(1-allyl-1-isopropylbut-3-enyl)oxirane 4i (180 $\mathrm{mg}, 1.00 \mathrm{mmol}$ ) was treated with LTMP in $t$-BuOMe for $8 \mathrm{~h}$ to give bicyclic alcohol $\mathbf{5 i}$ (148 $\mathrm{mg}$, $0.82 \mathrm{mmol}, 82 \%)$ as a colorless oil, $R_{\mathrm{f}}=0.4\left(20 \% \mathrm{Et}_{2} \mathrm{O}\right.$ in petrol); IR (film) $3435 \mathrm{bs}(\mathrm{O}-\mathrm{H}), 3059 \mathrm{~m}$ (cyclopropane), 3029w, 2960s, 2872s (C-H), 1631m (C=C), 1465m, 1411w, 1386m, 1371m, 1342w, 1322w, 1180w, 1077s, 1057s, 1028m; ${ }^{1} \mathrm{H}$ NMR (400 MHz) $0.05-0.08$ (m, 1H, H-6 endo), 0.85 (d, $\left.3 \mathrm{H}, \mathrm{CH}_{3}, J=6.8\right), 0.89-0.96$ (m, 4H, $\left.\mathrm{CH}_{3}, \mathrm{H}-6_{\text {exo }}\right), 1.19$ - 1.34 (m, 3H, H-1, H-5, H-4), 1.51 (sept, $1 \mathrm{H}, \mathrm{H}-10, J=6.8), 1.90(\mathrm{~d}, 1 \mathrm{H}, \mathrm{OH}, J=6.0), 2.01\left(\mathrm{dd}, 1 \mathrm{H}, \mathrm{H}-4^{\prime}, J=6.8,14.0\right), 2.15(\mathrm{dd}, 1 \mathrm{H}, \mathrm{H}-7, J$ $=6.8,14.0), 2.41\left(\mathrm{dd}, 1 \mathrm{H}, \mathrm{H}^{\prime} 7^{\prime}, J=7.6,13.6\right), 3.72(\mathrm{~d}, 1 \mathrm{H}, \mathrm{H}-2, J=5.6), 5.09(\mathrm{~d}, 1 \mathrm{H}, \mathrm{H}-9, J=$ 10.0), 5.18 (d, 1H, H-9', $J=16.8), 6.15-6.26(\mathrm{~m}, 1 \mathrm{H}, \mathrm{H}-8) ;{ }^{13} \mathrm{C}$ NMR (100 MHz) 17.3 (C-5), 18.0 $\left(\mathrm{CH}_{3}\right), 18.4\left(\mathrm{CH}_{3}\right), 19.0(\mathrm{C}-6), 27.5(\mathrm{C}-1), 34.9(\mathrm{C}-7), 36.4(\mathrm{C}-10), 38.8(\mathrm{C}-4), 60.3(\mathrm{C}-3), 82.8(\mathrm{C}-$ 2), $115.6(\mathrm{C}-9), 137.8(\mathrm{C}-8)$; $\mathrm{MS}(\mathrm{CI}+) \mathrm{m} / z: 198.2\left(\mathrm{MNH}_{4}{ }^{+} 10 \%\right), 180.2\left(\mathrm{MH}^{+}, 45 \%\right), 163.1(100 \%)$, $137.1(5 \%), 121.1(15 \%), 107.1(7 \%)$; HRMS $m / z: \mathrm{MNH}_{4}{ }^{+}$found $198.1852, \mathrm{C}_{12} \mathrm{H}_{24} \mathrm{NO}$ requires 198.1858.

\section{NOE study}

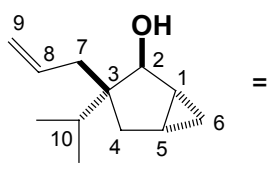

$5 \mathbf{i}$

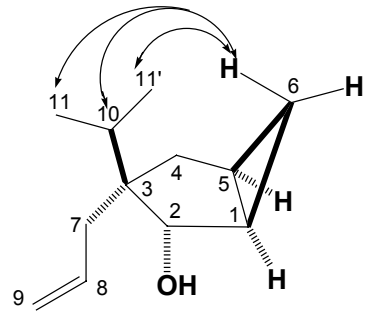

NOE study: irradiation of H- $6_{\text {endo }}, \mathrm{H}-10, \mathrm{H}-11$ and H-11' showed reciprocal signal enhancement, but no signal enhancement was observed between $\mathrm{H}-\mathrm{6}_{\text {endo }}$ and $\mathrm{H}-7 \mathrm{~s}$. 


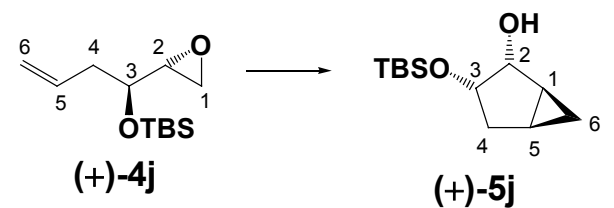

(2R,3S)-3-tert-butyldimethylsilyloxy-1,2-Epoxy-5-hexene (+)-4j

$\mathrm{Ti}(i-\mathrm{PrO})_{4}(0.41 \mathrm{~mL}, 1.4 \mathrm{mmol}, 0.1$ equiv. $)$ was added to a stirred solution of 1,5-hexadien-3-ol (1.37 g, $14.0 \mathrm{mmol}),(+)$-dicyclohexyl tartrate $(717 \mathrm{mg}, 2.1 \mathrm{mmol}, 0.15$ equiv.) and powdered $4 \AA$ molecular sieves $(627 \mathrm{mg})$ in $\mathrm{CH}_{2} \mathrm{Cl}_{2}(16 \mathrm{~mL})$ at $-20{ }^{\circ} \mathrm{C}$. After $30 \mathrm{~min}$, the mixture was treated dropwise with $t$-BuOOH (6.0 $\mathrm{M}$ in decane, $1.4 \mathrm{~mL}, 8.4 \mathrm{mmol}, 0.6$ equiv.), and then stirred at the same termperature for a further $19 \mathrm{~h}$. The reaction mixture was poured into a solution of iron (III) sulfate heptahydrate $(5.17 \mathrm{~g})$ and citric acid $(1.72 \mathrm{~g})$ in water $(16 \mathrm{~mL})$ at $0{ }^{\circ} \mathrm{C}$, then stirred for 30 min. The reaction was filtered through a pad of celite. The layers were separated and the aqueous layer extracted with $\mathrm{CH}_{2} \mathrm{Cl}_{2}(2 \times 30 \mathrm{~mL})$. The combined organic layers were dried $\left(\mathrm{MgSO}_{4}\right)$, filtered and concentrated to give the crude product. Purification by column chromatography (30\% $\mathrm{Et}_{2} \mathrm{O}$ in petrol) gave $(2 R, 3 S)-1,2-$-epoxy-3-hydroxy-5-hexene ${ }^{[11]}(1.02 \mathrm{~g}$, assumed to be $7.0 \mathrm{mmol}$, $50 \%$ yield) as a cloudy oil. ${ }^{1} \mathrm{H}$ NMR analysis of the product showed the presence of a significant amount of (+)-dicyclohexyl tartrate, and was used without further purification.

tert-Butyldimethylsilyl chloride $(2.11 \mathrm{~g}, 14.0 \mathrm{mmol}, 2.0$ equiv.) was added to a stirred solution of $(2 R, 3 S)$-1,2-epoxy-3-hydroxy-5-hexene $(7.0 \mathrm{mmol})$ and imidazole (1.19 g, $17.5 \mathrm{mmol}, 2.5$ equiv.) in $\mathrm{CH}_{2} \mathrm{Cl}_{2}(60 \mathrm{~mL})$ at $\mathrm{rt}$ for $5 \mathrm{~h}$. The reaction mixture was washed with sat. aq. $\mathrm{NaHCO}_{3}(50 \mathrm{~mL})$, the layers were separated and the aqueous layer extracted with $\mathrm{CH}_{2} \mathrm{Cl}_{2}(2 \times 20 \mathrm{~mL})$. The combined organic layers were dried $\left(\mathrm{MgSO}_{4}\right)$, filtered and concentrated to give the crude product. Purification by column chromatography $\left(10 \% \mathrm{Et}_{2} \mathrm{O}\right.$ in petrol) gave $(2 R, 3 S)$-3-tert-butyldimethylsilyloxy-1,2epoxy-5-hexene (+)-4j (1.166 g, $5.11 \mathrm{mmol}, 37 \%$ from 1,5-hexadien-3-ol) as a colorless oil, $R_{\mathrm{f}}=0.5$ $\left(10 \% \mathrm{Et}_{2} \mathrm{O}\right.$ in petrol), $[\alpha]_{\mathrm{D}}^{25}=+32.4^{\circ}\left(c=1.0\right.$, in $\left.\mathrm{CHCl}_{3}\right), e e=97 \%$; IR (film) $3079 \mathrm{w}$ (epoxide), 2930s, 2858s (C-H), 1643w, 1473m, 1362w, 1253s, 1112s; ${ }^{1} \mathrm{H}$ NMR (400 MHz) 0.05 (s, 6H, $2 \times$ $\left.\mathrm{SiCH}_{3}\right), 0.89\left(\mathrm{~s}, 9 \mathrm{H}, \mathrm{SiC}\left(\mathrm{CH}_{3}\right)_{3}\right), 2.29-2.43(\mathrm{~m}, 2 \mathrm{H}, 2 \times \mathrm{H}-4), 2.67$ (dd, 1H, H-1, J = 2.8, 5.6), 2.71 
(dd, 1H, H-1' $, J=3.6,5.2), 2.90-2.93$ (m, 1H, H-2), 3.61 - 3.65 (m, 1H, H-3), 5.07 - 5.14 (m, 2H, $2 \times \mathrm{H}-6), 5.82-5.93(\mathrm{~m}, 1 \mathrm{H}, \mathrm{H}-5) ;{ }^{13} \mathrm{C} \mathrm{NMR}(100 \mathrm{MHz})-4.8\left(\mathrm{SiCH}_{3}\right),-4.5\left(\mathrm{SiCH}_{3}\right), 18.1$ $\left(\mathrm{SiC}\left(\mathrm{CH}_{3}\right)_{3}\right), 25.8\left(\mathrm{SiC}\left(\mathrm{CH}_{3}\right)_{3}\right), 40.0(\mathrm{C}-4), 44.8(\mathrm{C}-1), 54.3(\mathrm{C}-2), 71.0(\mathrm{C}-3), 117.4(\mathrm{C}-6), 134.2(\mathrm{C}-$ 5); MS (CI+) m/z: $229.2\left(\mathrm{MH}^{+}, 38 \%\right), 213.1(20 \%), 199.2(9 \%), 187.1(48 \%), 171.1(100 \%), 141.1$ (55\%), 129.0 (87\%), 117.0 (18\%); HRMS $m / z$ : found $229.1622 \mathrm{C}_{12} \mathrm{H}_{25} \mathrm{O}_{2}$ Si required 229.1624.

\section{(2R,3S)-3-tert-Butyldimethylsilanyloxybicyclo[3.1.0] hexan-2-ol (+)-5j}

Following the typical cyclopropanation procedure, $(2 R, 3 S)$-3-tert-butyldimethylsilyloxy-1,2-epoxy5-hexene (+)-4j (228 mg, $1.00 \mathrm{mmol})$ was treated with LTMP in $t$-BuOMe for $16 \mathrm{~h}$ to give $(2 R, 3 S)$ 3-tert-butyldimethylsilanyloxybicyclo[3.1.0]hexan-2-ol (+)-5j (139 mg, $0.61 \mathrm{mmol}, 61 \%)$ as a colorless oil, $R_{\mathrm{f}}=0.4\left(10 \% \mathrm{Et}_{2} \mathrm{O}\right.$ in petrol $) ;[\alpha]_{\mathrm{D}}^{25}=+1.0^{\circ}\left(c=1.0\right.$, in $\left.\mathrm{CHCl}_{3}\right), e e=96 \%$; IR (film) 3544bs (O-H), 2931s, 2859s (C-H), 1472m, 1362m, 1253s, 1174m, 1118s, 1086s (Si-O), 1049m, 1019m; ${ }^{1} \mathrm{H}$ NMR (400 MHz) $0.06\left(\mathrm{~s}, 6 \mathrm{H}, 2 \times \mathrm{SiCH}_{3}\right), 0.08-0.11$ (m, 1H, H-6 endo), $0.43-0.49$ (m, 1H, H-6 exo), 0.89 (s, 9H, $\left.\mathrm{SiC}\left(\mathrm{CH}_{3}\right)_{3}\right), 1.35$ - 1.41 (m, 1H, H-5), 1.50 - 1.54 (m, 1H, H-1), $1.74-1.80$ (m, 1H, H-4), 1.96 (dd, 1H, H-4' $, J=7.2,12.4), 2.93$ (s, 1H, OH), 3.78 - 3.83 (m, 1H, H-3), 3.91 (d, $1 \mathrm{H}, \mathrm{H}-2, J=4.8) ;{ }^{13} \mathrm{C}$ NMR $(100 \mathrm{MHz})-5.1\left(\mathrm{SiCH}_{3}\right),-4.7\left(\mathrm{SiCH}_{3}\right), 7.4(\mathrm{C}-6), 15.6(\mathrm{C}-5), 18.0$ $\left(\mathrm{SiC}\left(\mathrm{CH}_{3}\right)_{3}\right), 21.0(\mathrm{C}-1), 25.7\left(\mathrm{SiC}\left(\mathrm{CH}_{3}\right)_{3}\right), 33.4(\mathrm{C}-4), 72.3(\mathrm{C}-3), 72.4(\mathrm{C}-2)$; MS (CI+) $m / z: 229.2$ $\left(\mathrm{MH}^{+}, 6 \%\right), 211.1(100 \%), 187.1(10 \%), 171.1(93 \%), 153.1(6 \%), 132.1(7 \%), 115.1(10 \%), 105.0$ (13\%); HRMS $m / z$ : $\mathrm{MH}^{+}$found $229.1618 \mathrm{C}_{12} \mathrm{H}_{25} \mathrm{O}_{2}$ Si required 229.1624.

\section{Procedures for the formation of bicyclic alcohols $5 k-0$ and $18 a-b$ from epoxides 4k-o and LTMP (Table 2 in main paper)}

exo-6-Vinylbicyclo[3.1.0]hexan-2-ol $5 \mathrm{k}$ and (2-buta-1,3-dienyl-cyclopropyl)-methanol $9(\mathrm{R}=$ $\mathrm{CH}=\mathrm{CH}_{2}$ )

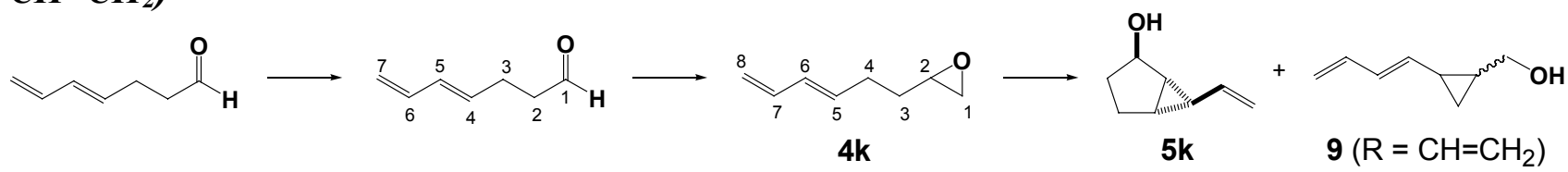

\section{1,2-Epoxy-5,7-octadiene $4 \boldsymbol{k}$}

DIBALH (1.0 M in hexane, $2.9 \mathrm{~mL}, 2.9 \mathrm{mmol}, 1.0$ equiv.) was added slowly to a stirred solution of methyl (4E)-hepta-4,6-dienoate (400 mg, $2.85 \mathrm{mmol})$ in $\mathrm{CH}_{2} \mathrm{Cl}_{2}(12 \mathrm{~mL})$ at $-78{ }^{\circ} \mathrm{C}$. After $1 \mathrm{~h}$, the 
mixture was treated slowly with $\mathrm{MeOH}$ ( 2 drops) and then warmed to rt. The mixture was washed with water $(30 \mathrm{~mL})$ and the layers were separated. The organic layer was dried $\left(\mathrm{MgSO}_{4}\right)$, filtered and concentrated to give the crude product. Purification by column chromatography $\left(10 \% \mathrm{Et}_{2} \mathrm{O}\right.$ in petrol) gave (4E)-hepta-4,6-dienal ( $228 \mathrm{mg}, 2.07 \mathrm{mmol}, 73 \%)$ as a colorless oil, $R_{\mathrm{f}}=0.2\left(10 \% \mathrm{Et}_{2} \mathrm{O}\right.$ in petrol); IR (film) 3086w, 3009w, 2913w, 2826w (C-H), 2720w, 1725s (C=O), 1653w (C=C), 1600w, 1446w, 1412w, 1388w, 1008m; ${ }^{1} \mathrm{H}$ NMR (400 MHz) $2.40-2.45$ (m, 2H, $\left.2 \times \mathrm{H}-3\right), 2.53-$ $2.58(\mathrm{~m}, 2 \mathrm{H}, 2 \times \mathrm{H}-2), 5.00(\mathrm{~d}, 1 \mathrm{H}, \mathrm{H}-7, J=10.0), 5.12\left(\mathrm{~d}, 1 \mathrm{H}, \mathrm{H}-7^{\prime}, J=17.2\right), 5.65-5.73(\mathrm{~m}, 1 \mathrm{H}$, H-4), $6.06-6.12$ (m, 1H, H-5), $6.24-6.34$ (m, 1H, H-6), 9.78 (t, 1H, H-1, J=1.2); ${ }^{1} \mathrm{H}$ NMR (100 MHz) 25.0 (C-3), 43.1 (C-2), 115.9 (C-7), 132.1 (C-5), 132.3 (C-4), 136.7 (C-6), 201.7 (C=O); MS (CI+) $m / z: 128.2\left(\mathrm{MNH}_{4}^{+}, 100 \%\right), 112.6(35 \%)$; HRMS $m / z: \mathrm{MNH}_{4}{ }^{+}$found $128.1925, \mathrm{C}_{7} \mathrm{H}_{14} \mathrm{NO}$ requires 128.1922 .

$n$-BuLi (1.6 M in hexane, $4.6 \mathrm{~mL}, 7.32 \mathrm{mmol}, 1.05$ equiv.) was added dropwise ( 1 drop/2 s) to a stirred solution of (4E)-hepta-4,6-dienal $(768 \mathrm{mg}, 6.97 \mathrm{mmol})$ and dibromomethane $(0.74 \mathrm{~mL}, 10.50$ mmol, 1.5 equiv. $)$ in THF $(28 \mathrm{~mL})$ at $-78^{\circ} \mathrm{C}$. The resulting mixture was warmed to $\mathrm{rt}$ and stirred for $15 \mathrm{~h}$. The reaction mixture was washed with sat. aq. $\mathrm{NH}_{4} \mathrm{Cl}(50 \mathrm{~mL})$, the layers were separated and then the aqueous layer extracted with $\mathrm{Et}_{2} \mathrm{O}(2 \times 40 \mathrm{~mL})$. The combined organic layers were dried $\left(\mathrm{MgSO}_{4}\right)$, filtered and concentrated to give the crude product. Purification by column chromatography $\left(10 \% \mathrm{Et}_{2} \mathrm{O}\right)$ gave epoxide $4 \mathbf{k}(579 \mathrm{mg}, 4.66 \mathrm{mmol}, 67 \%)$ as a colorless oil, $R_{\mathrm{f}}=0.6$ (10\% $\mathrm{Et}_{2} \mathrm{O}$ in petrol); IR (film) 3038w (epoxide), 2990s, 2922s, 2855w (C-H), 1715w, 1648w, 1600w, 1480w, 1441m, 1412m, 1258w (epoxide), 1133w, 1003s; ${ }^{1} \mathrm{H}$ NMR (400 MHz) $1.61-1.68$ (m, 2H, $2 \times \mathrm{H}-3), 2.21-2.32(\mathrm{~m}, 2 \mathrm{H}, 2 \times \mathrm{H}-4), 2.48-2.50(\mathrm{~m}, 1 \mathrm{H}, \mathrm{H}-1), 2.75-2.77$ (m, 1H, H-1', $2.91-2.96(\mathrm{~m}, 1 \mathrm{H}, \mathrm{H}-2), 4.99(\mathrm{~d}, 1 \mathrm{H}, \mathrm{H}-8, J=10.0), 5.10\left(\mathrm{~d}, 1 \mathrm{H}, \mathrm{H}-8^{\prime}, J=16.8\right), 5.69$ - $5.76(\mathrm{~m}$, $1 \mathrm{H}, \mathrm{H}-5), 6.10(\mathrm{dd}, 1 \mathrm{H}, \mathrm{H}-6, J=10.4,15.2), 6.31(\mathrm{dt}, 1 \mathrm{H}, \mathrm{H}-7, J=10.4,16.8) ;{ }^{13} \mathrm{C} \mathrm{NMR}(100 \mathrm{MHz})$ 29.0 (C-4), 32.0 (C-3), 47.1 (C-1), 51.8 (C-2), 115.4 (C-8), 131.7 (C-6), 133.6 (C-5), 136.9 (C-7); MS (CI+) m/z: $142.1230\left(\mathrm{MNH}_{4}^{+} 100 \%\right), 125.0946\left(\mathrm{MH}^{+}, 50 \%\right), 107.0878(20 \%) ; \mathrm{HRMS} m / z$ : $\mathrm{MNH}_{4}{ }^{+}$found 142.1230, $\mathrm{C}_{8} \mathrm{H}_{16} \mathrm{NO}$ requires 142.1232. 
exo-6-Vinylbicyclo[3.1.0]hexan-2-ol $\mathbf{5 k}$ and (2-buta-1,3-dienyl-cyclopropyl)-methanol $9(R=$ $\mathrm{CH}=\mathrm{CH}_{2}$ )

Following the typical cyclopropanation procedure, 1,2-epoxy-5,7-octadiene $4 \mathbf{k}$ (124 mg, $1.00 \mathrm{mmol})$ was treated with LTMP in $t$-BuOMe for $16 \mathrm{~h}$ to give an inseparable 1:1.5 mixture of bicyclic alcohol 5k and cyclopropane $9\left(\mathrm{R}=\mathrm{CH}=\mathrm{CH}_{2}\right)(80 \mathrm{mg}, 0.65 \mathrm{mmol}, 65 \%)$ as a light yellow oil, $R_{\mathrm{f}}=0.5(50 \%$ $\mathrm{Et}_{2} \mathrm{O}$ in petrol). Ratio determined in ${ }^{1} \mathrm{H}$ NMR using the $\mathrm{CHOH}$ signal of $\mathbf{5 r}$ and $\mathrm{CH}_{2} \mathrm{OH}$ signal of 9 $\left(\mathrm{R}=\mathrm{CH}=\mathrm{CH}_{2}\right)$.

\section{exo-4,4-Dimethyl-6-vinylbicyclo[3.1.0]hexan-2-ol 5l}

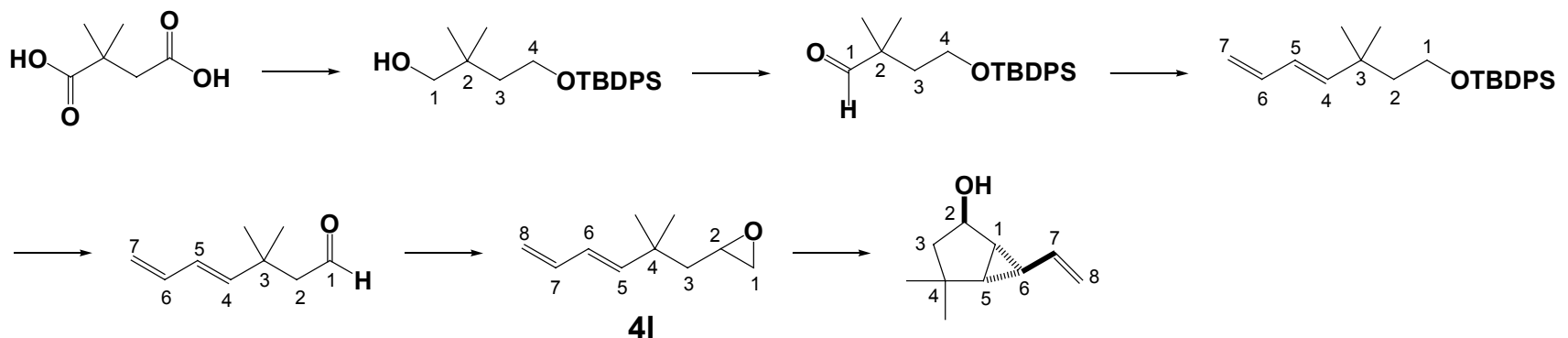

5!

(5E)-1,2-Epoxy-4,4-dimethylocta-3,5-diene $4 \boldsymbol{l}$

$\mathrm{LiAlH}_{4}(2.3 \mathrm{M}$ in THF, $52.2 \mathrm{~mL}, 120 \mathrm{mmol}, 6.0$ equiv.) was added slowly to a stirred solution of 2,2-dimethylsuccinic acid $(2.92 \mathrm{~g}, 20.0 \mathrm{mmol})$ in THF $(250 \mathrm{~mL})$ at $-78{ }^{\circ} \mathrm{C}$ and stirred at $\mathrm{rt}$ for $5 \mathrm{~h}$. The reaction mixture was cooled to $-78{ }^{\circ} \mathrm{C}$ and quenched by sequential addition of water $(4.6 \mathrm{~mL})$, aq. $\mathrm{NaOH}(15 \mathrm{w} \%, 4.6 \mathrm{~mL})$ and water $(13.8 \mathrm{~mL})$. The solid precipitates formed were filtered and washed through with $\mathrm{Et}_{2} \mathrm{O}(2 \times 150 \mathrm{~mL})$. The filtrate was dried $\left(\mathrm{MgSO}_{4}\right)$, filtered and concentrated to give the 2,2-dimethyl-butane-1,4-diol (2.33 g, $19.7 \mathrm{mmol}, 99 \%)$ as a coulourless oil, which was sufficiently pure to be used without further purification. tert-Butyldiphenylsilyl chloride $(0.52 \mathrm{~mL}$, $2.0 \mathrm{mmol}, 1.0$ equiv.) was added to a stirred solution of 2,2-dimethyl-butane-1,4-diol (236 mg, 2.0 mmol) and imidazole (204 mg, $3.0 \mathrm{mmol}, 1.5$ equiv.) in anhydrous DMF (5 mL) at rt. After $20 \mathrm{~h}$, the mixture was diluted with $\mathrm{Et}_{2} \mathrm{O}(20 \mathrm{~mL})$ washed with sat. aq. $\mathrm{NaHCO}_{3}(25 \mathrm{~mL})$, the layers were separated and then the aqueous layer extracted with $\mathrm{Et}_{2} \mathrm{O}(20 \mathrm{~mL})$. The combined organic layers were dried $\left(\mathrm{MgSO}_{4}\right)$, filtered and concentrated to give the crude product. Purification by column chromatography $\left(30 \% \mathrm{Et}_{2} \mathrm{O}\right.$ in petrol) gave 4-(tert-butyldiphenylsilyloxy)-2,2-dimethylbutan-1-ol (522 mg, $1.46 \mathrm{mmol}, 73 \%)$ as a colorless oil, $R_{\mathrm{f}}=0.3\left(30 \% \mathrm{Et}_{2} \mathrm{O}\right.$ in petrol); IR (film) $3431 \mathrm{bs}(\mathrm{O}-\mathrm{H})$, 
3072m, 2957s (C-H), 1472s, 1428s, 1391m, 1363m, 1111s (Si-O), 1056s; ${ }^{1} \mathrm{H}$ NMR (400 MHz) $0.92\left(\mathrm{~s}, 6 \mathrm{H}, 2 \times \mathrm{CH}_{3}\right), 1.08\left(\mathrm{~s}, 9 \mathrm{H}, \mathrm{SiC}\left(\mathrm{CH}_{3}\right)_{3}\right), 1.57(\mathrm{t}, 2 \mathrm{H}, 2 \times \mathrm{H}-3, J=4.0), 3.40(\mathrm{~d}, 2 \mathrm{H}, 2 \times \mathrm{H}-1$, $J=4.0), 3.74(\mathrm{t}, 2 \mathrm{H}, 2 \times \mathrm{H}-4, J=4.0), 7.40-7.48(\mathrm{~m}, 6 \mathrm{H}, 6 \times \mathrm{Ar}-\mathrm{H}), 7.69-7.71(\mathrm{~m}, 4 \mathrm{H}, 4 \times \mathrm{Ar}-\mathrm{H})$; ${ }^{13} \mathrm{C}$ NMR $(100 \mathrm{MHz}) 19.0\left(\mathrm{SiC}\left(\mathrm{CH}_{3}\right)_{3}\right), 25.0\left(\mathrm{CH}_{3}\right), 26.8\left(\mathrm{SiC}\left(\mathrm{CH}_{3}\right)_{3}\right), 26.0(\mathrm{C}-2), 42.0(\mathrm{C}-3), 61.1$ (C-4), 71.6 (C-1), 127.8 (Ar-C), 129.8 (Ar-C), 133.0 (Ar-C), 135.5 (Ar-C); MS (CI+) m/z: 357.2 $\left(\mathrm{MH}^{+}, 3 \%\right), 279.2$ (16\%), 229.1 (11\%), 216.1 (4\%), 199.1 (100\%), 181.0 (11\%); HRMS m/z: $\mathrm{MH}^{+}$ found 357.2233, $\mathrm{C}_{22} \mathrm{H}_{33} \mathrm{O}_{2}$ Si requires 357.2250.

DMSO (3.0 mL, $42.9 \mathrm{mmol}, 2.5$ equiv.) was added slowly to a stirred solution of oxalyl chloride (1.6 mL, $18.9 \mathrm{mmol}, 1.1$ equiv.) in $\mathrm{CH}_{2} \mathrm{Cl}_{2}(80 \mathrm{~mL})$ at $-78{ }^{\circ} \mathrm{C}$ and stirred for $5 \mathrm{~min}$. The mixture was treated slowly with a solution of 4-(tert-butyldiphenylsilyloxy)-2,2-dimethylbutan-1-ol (6.12 g, $17.2 \mathrm{mmol})$ in $\mathrm{CH}_{2} \mathrm{Cl}_{2}(20 \mathrm{~mL})$, stirred for $15 \mathrm{~min}$ and then treated slowly with $\mathrm{Et}_{3} \mathrm{~N}(12.0 \mathrm{~mL}, 86.0$ mmol, 5.0 equiv.). After $5 \mathrm{~min}$, the resulting mixture was warmed to $\mathrm{rt}$ and stirred for a further $12 \mathrm{~h}$. The reaction mixture was washed with water $(100 \mathrm{~mL})$, the layers were separated and then the aqueous layer extracted with $\mathrm{CH}_{2} \mathrm{Cl}_{2}(2 \times 100 \mathrm{~mL})$. The combined organic layers were dried $\left(\mathrm{MgSO}_{4}\right)$, filtered and concentrated to give the crude product. Purification by column chromatography $\left(10 \% \quad \mathrm{Et}_{2} \mathrm{O} \quad\right.$ in petrol $)$ gave 4-(tert-butyldiphenylsilyloxy)-2,2dimethylbutyraldehyde $(5.55 \mathrm{~g}, 15.7 \mathrm{mmol}, 91 \%)$ as a colorless oil, $R_{\mathrm{f}}=0.5\left(10 \% \mathrm{Et}_{2} \mathrm{O}\right.$ in petrol); IR (film) 3072m, 2932s (C-H), 2707m, 1732s (C=O), 1472s, 1428s, 1391m, 1363m, 1188w, 1111s (Si-O), 1053m; ${ }^{1} \mathrm{H}$ NMR (400 MHz) 1.07 (s, 9H, $\left.\mathrm{SiC}\left(\mathrm{CH}_{3}\right)_{3}\right), 1.10\left(\mathrm{~s}, 6 \mathrm{H}, 2 \times \mathrm{CH}_{3}\right), 1.83(\mathrm{t}, 2 \mathrm{H}, 2 \times$ $\mathrm{H}-3, J=4.0), 3.68(\mathrm{t}, 2 \mathrm{H}, 2 \times \mathrm{H}-4, J=4.0), 7.40-7.48(\mathrm{~m}, 6 \mathrm{H}, \mathrm{Ar}-\mathrm{H}), 7.68-7.70(\mathrm{~m}, 4 \mathrm{H}, \mathrm{Ar}-\mathrm{H})$, 9.59 (s, 1H, CHO); ${ }^{1} \mathrm{H}$ NMR (100 MHz) $19.0\left(\mathrm{SiC}\left(\mathrm{CH}_{3}\right)_{3}\right), 21.5\left(\mathrm{CH}_{3}\right), 26.7\left(\mathrm{SiC}\left(\mathrm{CH}_{3}\right)_{3}\right), 40.6(\mathrm{C}-$ 3), 44.5 (C-2), 60.1 (C-4), 127.6 (Ar-C), 129.6 (Ar-C), 133.3 (Ar-C), 135.5 (Ar-C), 205.3 (C=O); MS (CI+) m/z: $372.2\left(\mathrm{MNH}_{4}^{+}, 2 \%\right), 355.2\left(\mathrm{MH}^{+}, 86 \%\right), 325.2(5 \%), 297.1(83 \%), 277.2(23 \%)$, 267.1 (12\%), 216.1 (11\%), 199.1 (28\%), 148.1 (24\%); HRMS $m / z: \mathrm{MNH}_{4}^{+}$found 372.2364, $\mathrm{C}_{22} \mathrm{H}_{34} \mathrm{NO}_{2} \mathrm{Si}$ requires 372.2359 . 
$n$-BuLi (1.6 M in hexane, $8.7 \mathrm{~mL}, 13.9 \mathrm{mmol}, 1.2$ equiv.) was added to a stirred solution of allyl diethyl phosphonate $(2.47 \mathrm{~g}, 13.9 \mathrm{mmol}, 1.2$ equiv. $)$ in THF $(85 \mathrm{~mL})$ at $-78^{\circ} \mathrm{C}^{[12]}$ and stirred for 15 min. The mixture was treated slowly with a solution of 4-(tert-butyldiphenylsilyloxy)-2,2dimethylbutyraldehyde (4.10 g, $11.6 \mathrm{mmol})$ and HMPA (4.0 mL, $23.2 \mathrm{mmol}, 2.0$ equiv.) in THF (10 $\mathrm{mL}$ ), and then stirred for $2 \mathrm{~h}$. The resulting mixture was warmed to rt, and then stirred for a further $12 \mathrm{~h}$. The reaction mixture was washed with aq. $\mathrm{HCl}(1 \mathrm{~N}, 200 \mathrm{~mL})$, the layers were separated and then the aqueous layer extracted with $\mathrm{Et}_{2} \mathrm{O}(2 \times 100 \mathrm{~mL})$. The combined organic layers were dried $\left(\mathrm{MgSO}_{4}\right)$, filtered and concentrated to give the crude product. Purification by column chromatography $\left(10 \% \quad \mathrm{Et}_{2} \mathrm{O}\right.$ in petrol) gave (4E)-tert-butyl-(3,3-dimethylhepta-4,6dienyloxy)diphenylsilane $(3.73 \mathrm{~g}, 9.85 \mathrm{mmol}, 85 \%)$ as a colorless oil, $R_{\mathrm{f}}=0.7\left(10 \% \mathrm{Et}_{2} \mathrm{O}\right.$ in petrol); IR (film) 3071m, 2960s (C-H), 2858s, 1649w, 1602w, 1472m, 1390m, 1362m, 1188w, 1111s (Si-O), 1044m, 1005s; ${ }^{1} \mathrm{H}$ NMR (400 MHz) $1.00\left(\mathrm{~s}, 6 \mathrm{H}, 2 \times \mathrm{CH}_{3}\right), 1.07\left(\mathrm{~s}, 9 \mathrm{H},\left(\mathrm{SiC}\left(\mathrm{CH}_{3}\right)_{3}\right), 1.66(\mathrm{t}, 2 \mathrm{H}, 2 \times\right.$ $\mathrm{H}-2, J=8.0), 3.69(\mathrm{t}, 2 \mathrm{H}, 2 \times \mathrm{H}-1, J=8.0), 4.97(\mathrm{~d}, 1 \mathrm{H}, \mathrm{H}-7, J=12.0), 5.10\left(\mathrm{~d}, 1 \mathrm{H}, \mathrm{H}-7^{\prime}, J=20.0\right)$, $5.62(\mathrm{~d}, 1 \mathrm{H}, \mathrm{H}-4, J=16.0), 5.93(\mathrm{dd}, 1 \mathrm{H}, \mathrm{H}-5, J=12.0,16.0), 6.23-6.30(\mathrm{~m}, 1 \mathrm{H}, \mathrm{H}-6) ;{ }^{13} \mathrm{C}$ NMR $(100 \mathrm{MHz}) 19.1\left(\mathrm{SiC}\left(\mathrm{CH}_{3}\right)_{3}\right), 26.8\left(\mathrm{SiC}\left(\mathrm{CH}_{3}\right)_{3}\right), 27.5\left(\mathrm{CH}_{3}\right), 35.1(\mathrm{C}-3), 45.1(\mathrm{C}-2), 61.2(\mathrm{C}-1)$, 114.8 (C-7), 126.8 (C-5), 127.6 (Ar-C), 129.5 (Ar-C), 134.0 (C-6), 135.6 (Ar-C), 137.6 (Ar-C), 144.7 (C-5); $\mathrm{MS}(\mathrm{CI}+)$ m/z: $379.2\left(\mathrm{MH}^{+}, 100 \%\right), 321.2$ (59\%), 199.1 (21\%), 148.1 (100\%), 123.1 (61\%); HRMS $m / z$ : $\mathrm{MH}^{+}$found 379.2452, $\mathrm{C}_{22} \mathrm{H}_{35} \mathrm{OSi}$, requires 379.2457.

TBAF (1.0 M in THF, $19.7 \mathrm{~mL}, 19.7 \mathrm{mmol}, 2.0$ equiv.) was added to a stirred solution of (4E)-tertbutyl-(3,3-dimethylhepta-4,6-dienyloxy)diphenylsilane (3.73 g, $9.85 \mathrm{mmol})$ in THF $(20 \mathrm{~mL})$ at $\mathrm{rt}$. After $12 \mathrm{~h}$, the mixture was poured into water $(80 \mathrm{~mL})$ and the aqueous layer extracted with $\mathrm{Et}_{2} \mathrm{O}$ $(80 \mathrm{~mL})$. The combined organic layers were dried $\left(\mathrm{MgSO}_{4}\right)$, filtered and concentrated to give $(4 E)$ 3,3-dimethylhepta-4,6-dien-1-ol (1.43 g, $10.2 \mathrm{mmol})$ as a colorless oil, which was sufficiently pure to be used without further purification. DMSO (1.8 mL, $25.5 \mathrm{mmol}, 2.5$ equiv.) was added slowly to a stirred solution of oxalyl chloride $\left(1.1 \mathrm{~mL}, 11.2 \mathrm{mmol}, 1.1\right.$ equiv.) in $\mathrm{CH}_{2} \mathrm{Cl}_{2}(40 \mathrm{~mL})$ at $-78{ }^{\circ} \mathrm{C}$ and stirred for $5 \mathrm{~min}$. The mixture was treated slowly with a solution of the above alcohol (1.43 $\mathrm{g}$, $10.2 \mathrm{mmol})$ in $\mathrm{CH}_{2} \mathrm{Cl}_{2}(10 \mathrm{~mL})$, stirred for a $15 \mathrm{~min}$ and then treated slowly with $\mathrm{Et}_{3} \mathrm{~N}$ (7.1 mL, 51.0 
mmol, 5.0 equiv.). After $5 \mathrm{~min}$, the mixture was warmed to $\mathrm{rt}$ and stirred for a further $5 \mathrm{~h}$. The reaction mixture was washed with water $(100 \mathrm{~mL})$, the layers were separated and then the aqueous layer extracted with $\mathrm{CH}_{2} \mathrm{Cl}_{2}(2 \times 100 \mathrm{~mL})$. The combined organic layers were dried $\left(\mathrm{MgSO}_{4}\right)$, filtered and concentrated to give the crude product. Purification by column chromatography $(10 \%$ $\mathrm{Et}_{2} \mathrm{O}$ in petrol) gave (4E)-3,3-dimethylhepta-4,6-dienal (1.20 g, $8.68 \mathrm{mmol}, 88 \%$ from (4E)-tertbutyl-(3,3-dimethylhepta-4,6-dienyloxy)diphenylsilane) as a colorless oil, $R_{\mathrm{f}}=0.5\left(10 \% \mathrm{Et}_{2} \mathrm{O}\right.$ in petrol); IR (film) 2964s (C-H), 2735w, 1722s (C=O), 1649w, 1603w, 1470w, 1386w, 1045w, 1007s; ${ }^{1} \mathrm{H}$ NMR (400 MHz) $1.18\left(\mathrm{~s}, 6 \mathrm{H}, 2 \times \mathrm{CH}_{3}\right), 2.36(\mathrm{~d}, 2 \mathrm{H}, 2 \times \mathrm{H}-2, J=2.8), 5.04(\mathrm{~d}, 1 \mathrm{H}, \mathrm{H}-7, J=$ 10.0), 5.17 (d, 1H, H-7' $J=16.9), 5.78(\mathrm{~d}, 1 \mathrm{H}, \mathrm{H}-4, J=15.2), 6.05$ (dd, 1H, H-5, $J=10.0,15.2)$, 6.27 - 6.37 (m, 1H, H-6); ${ }^{13} \mathrm{C}$ NMR (100 MHz) $27.6\left(\mathrm{CH}_{3}\right), 35.4(\mathrm{C}-3), 55.0(\mathrm{C}-2), 116.4(\mathrm{C}-7)$, 128.1 (C-5), 136.9 (C-6), 142.2 (C-4), 203.0 (C-1); $\mathrm{MS}(\mathrm{CI}+) \mathrm{m} / z: 156.1\left(\mathrm{MNH}_{4}^{+}, 91 \%\right), 148.1$ (76\%), 139.1 (100\%), $131.1(4 \%), 123.1$ (6\%), $109.1(10 \%)$; HRMS $m / z: \mathrm{MNH}_{4}{ }^{+}$found 156.1391, $\mathrm{C}_{9} \mathrm{H}_{18} \mathrm{NO}$, requires 156.1388 .

$n$-BuLi (1.6 M in hexane, $6.0 \mathrm{~mL}, 9.6 \mathrm{mmol}, 1.1$ equiv.) was added dropwise ( $1 \mathrm{drop} / 2 \mathrm{~s})$ to a stirred solution of (4E)-3,3-dimethylhepta-4,6-dienal (1.20 g, $8.68 \mathrm{mmol})$ and dibromomethane $(0.73$ $\mathrm{mL}, 10.4 \mathrm{mmol}, 1.2$ equiv.) in THF $(35 \mathrm{~mL})$ at $-78{ }^{\circ} \mathrm{C} .^{[6]}$ The resulting mixture was warmed to $\mathrm{rt}$ and stirred for $16 \mathrm{~h}$. The reaction mixture was washed with sat. aq. $\mathrm{NH}_{4} \mathrm{Cl}(40 \mathrm{~mL})$, the layers were separated and then the aqueous aqueous layer extracted with $\mathrm{Et}_{2} \mathrm{O}(2 \times 40 \mathrm{~mL})$. The combined organic layers were dried $\left(\mathrm{MgSO}_{4}\right)$, filtered and concentrated to give the crude product. Purification by column chromatography $\left(10 \% \mathrm{Et}_{2} \mathrm{O}\right)$ gave $(5 E)$-1,2-epoxy-4,4-dimethylocta-3,5-diene $4 \mathbf{l}$ (695 $\mathrm{mg}, 4.57 \mathrm{mmol}, 53 \%)$ as a colorless oil, $R_{\mathrm{f}}=0.4\left(10 \% \mathrm{Et}_{2} \mathrm{O}\right.$ in petrol); IR (film) $3086 \mathrm{w}$ (epoxide), 3039m, 2963s (C-H), 1649m, 1603w, 1469m, 1385m, 1365m, 1260w, 1190w, 1006s; ${ }^{1} \mathrm{H}$ NMR (400 MHz) $1.12\left(\mathrm{~s}, 3 \mathrm{H}, \mathrm{CH}_{3}\right), 1.14\left(\mathrm{~s}, 3 \mathrm{H}, \mathrm{CH}_{3}\right), 1.49-1.59(\mathrm{~m}, 2 \mathrm{H}, 2 \times \mathrm{H}-3), 2.40-2.42$ (m, 1H, H-1), $2.73\left(\mathrm{dd}, 1 \mathrm{H}, \mathrm{H}-1^{\prime}, J=4.0,8.0\right), 2.88-2.93(\mathrm{~m}, 1 \mathrm{H}, \mathrm{H}-2), 5.00(\mathrm{~d}, 1 \mathrm{H}, \mathrm{H}-8, J=12.0), 5.15(\mathrm{~d}, 1 \mathrm{H}$, H-8' $J=16.0), 5.74$ (d, 1H, H-5, $J=16.0), 6.04$ (dd, 1H, H-6, $J=12.0,16.0), 6.29$ - 6.38 (m, 1H, $\mathrm{H}-7) ;{ }^{13} \mathrm{C}$ NMR (100 MHz) $27.1\left(\mathrm{CH}_{3}\right), 27.7\left(\mathrm{CH}_{3}\right), 35.9$ (C-4), 45.5 (C-3), 46.8 (C-1), $49.5(\mathrm{C}-2)$, 115.5 (C-8), 127.4 (C-6), 137.3 (C-7), 143.9 (C-5); MS (CI+) m/z: $170.2\left(\mathrm{MNH}_{4}^{+}, 34 \%\right), 153.1$ 
(100\%), 148.1 (19\%), 135.1 (71\%), 119.1 (11\%), $109.0(16 \%)$; HRM $m / z: \mathrm{MNH}_{4}^{+}$found 170.1537 $\mathrm{C}_{10} \mathrm{H}_{20} \mathrm{NO}$, requires 170.1545 .

exo-4,4-Dimethyl-6-vinylbicyclo[3.1.0]hexan-2-ol 5l

Following the typical cyclopropanation procedure, (5E)-1,2-epoxy-4,4-dimethylocta-3,5-diene $4 \mathbf{I}$ (152 mg, $1.00 \mathrm{mmol}$ ) was treated with LTMP in $t$-BuOMe for $16 \mathrm{~h}$ to give bicyclic alcohol $\mathbf{5 l}$ (114 $\mathrm{mg}, 0.75 \mathrm{mmol}, 75 \%)$ as a colorless oil, $R_{\mathrm{f}}=0.5\left(50 \% \mathrm{Et}_{2} \mathrm{O}\right.$ in petrol); IR (film) $3357 \mathrm{bs}(\mathrm{O}-\mathrm{H})$, 3082w, 2954s (C-H), 2868m, 1638s, 1466m, 1364m, 1337m, 1151s, 1036m; ${ }^{1} \mathrm{H}$ NMR (400 MHz) $1.05\left(\mathrm{~s}, 3 \mathrm{H}, \mathrm{CH}_{3}\right), 1.08$ - 1.11 (m, 1H, H-6), 1.26 (s, 3H, $\left.\mathrm{CH}_{3}\right), 1.27$ - 1.44 (m, 3H, H-3, H-3', H-5), $1.51-1.52$ (m, 1H, H-1), 1.62 (bs, 1H, OH), 4.32 (d, 1H, H-2, J=4.0), 4.83 (dd, 1H, H-8, $J=4.0$, 12.0), 4.99 (d, 1H, H-8' $J=20.0), 5.31-5.41$ (m, 1H, H-7); ${ }^{13} \mathrm{C}$ NMR (100 MHz) 23.2 (C-6), 26.6 $\left(\mathrm{CH}_{3}\right), 30.4\left(\mathrm{CH}_{3}\right), 33.9(\mathrm{C}-1), 38.5$ (C-5), 39.1 (C-3), 46.3 (C-4), 75.1 (C-2), $111.4(\mathrm{C}-8), 139.5(\mathrm{C}-$ 7); $\mathrm{MS}(\mathrm{CI}+) \mathrm{m} / z: 170.2\left(\mathrm{MNH}_{4}^{+}, 3 \%\right), 152.1\left(\mathrm{MH}^{+}, 50 \%\right), 135.1(100 \%), 119.1(8 \%), 108.1(6 \%)$; HRMS $m / z: \mathrm{MNH}_{4}{ }^{+}$found $170.1544 \mathrm{C}_{10} \mathrm{H}_{20} \mathrm{NO}$, requires 170.1545.

\section{endo-4,4-Dimethyl-6-vinylbicyclo[3.1.0]hexan-2-ol 5m}
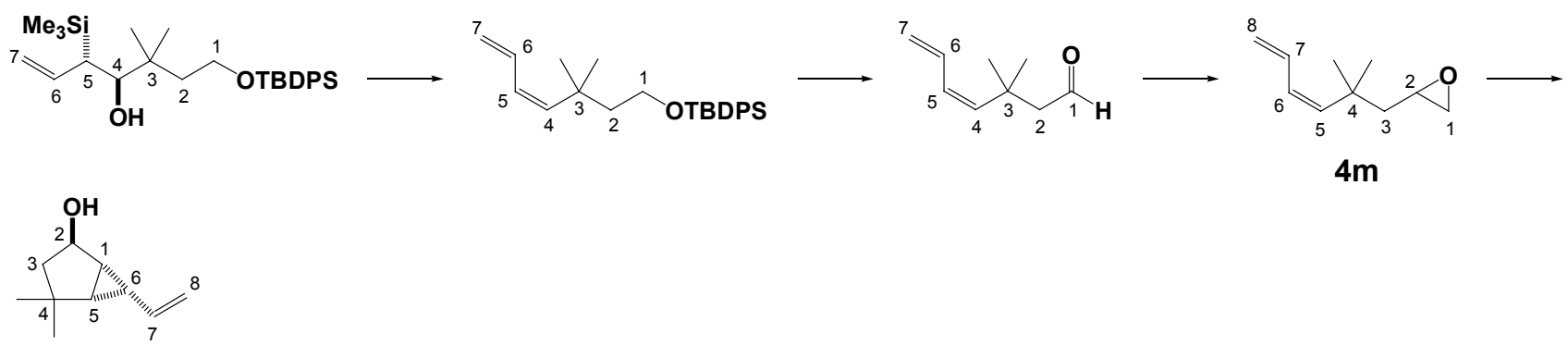

$5 \mathrm{~m}$

(5Z)-1,2-Epoxy-4,4-dimethylocta-5,7-diene 4m

$t$-BuLi (1.5 M in hexane, $2.2 \mathrm{~mL}, 3.3 \mathrm{mmol}, 1.0$ equiv.) was added to a stirred solution of allyl trimethylsilane $(377 \mathrm{mg}, 3.30 \mathrm{mmol})$ and TMEDA (0.5 mL, $3.3 \mathrm{mmol}, 1.0$ equiv.) in THF (11 mL) at $-78{ }^{\circ} \mathrm{C}$. The mixture was warmed to $-30{ }^{\circ} \mathrm{C}$, stirred for $2 \mathrm{~h}$ and then cooled to $-78{ }^{\circ} \mathrm{C}$. The mixture was treated with $\mathrm{Ti}(i-\mathrm{PrO})_{4}(1.0 \mathrm{~mL}, 3.3 \mathrm{mmol})$, stirred for $1 \mathrm{~h}$ and then treated slowly with a solution of 4-(tert-butyldiphenylsilyloxy)-2,2-dimethylbutyraldehyde (see synthesis of $\mathbf{4 l}, 1.06 \mathrm{~g}$, $3.0 \mathrm{mmol}$, ) in THF $(2 \mathrm{~mL})$. After $3.5 \mathrm{~h}$ at $-78{ }^{\circ} \mathrm{C}$, the mixture was quenched by slow addition of aq. 
$\mathrm{HCl}(2 \mathrm{M}, 10 \mathrm{~mL})$ and then warmed to rt. The layers were separated and the aqueous layer extracted with $\mathrm{Et}_{2} \mathrm{O}(40 \mathrm{~mL})$. The combine organic layers were dried $\left(\mathrm{MgSO}_{4}\right)$, filtered and concentrated to give the crude product. Purification by column chromatography $\left(5 \% \mathrm{Et}_{2} \mathrm{O}\right.$ in petrol) gave 1-(tert-butyldiphenylsilyloxy)-3,3-dimethyl-5-trimethylsilylhept-6-en-4-ol (1.00 g, $2.13 \mathrm{mmol}$, $71 \%)$ as a colorless oil; $R_{\mathrm{f}}=0.6\left(10 \% \mathrm{Et}_{2} \mathrm{O}\right.$ in petrol); IR (film) $3412 \mathrm{bs}(\mathrm{O}-\mathrm{H}), 3072 \mathrm{~s}, 2958 \mathrm{~s}(\mathrm{C}-\mathrm{H})$, 2892s, 1620m, 1472s, 1428s, 1390s, 1364m, 1245s, 1158s, 1112s (Si-O), 1029s; ${ }^{1} \mathrm{H}$ NMR (400 MHz) 0.07 (s, 9H, $\left.\mathrm{Si}\left(\mathrm{CH}_{3}\right)_{3}\right), 0.90\left(\mathrm{~s}, 3 \mathrm{H}, \mathrm{CH}_{3}\right), 0.95\left(\mathrm{~s}, 3 \mathrm{H}, \mathrm{CH}_{3}\right), 1.08\left(\mathrm{~s}, 9 \mathrm{H}, \mathrm{SiC}\left(\mathrm{CH}_{3}\right)_{3}\right), 1.40-$ $1.46(\mathrm{~m}, 1 \mathrm{H}, \mathrm{H}-2), 1.74-1.81\left(\mathrm{~m}, 1 \mathrm{H}, \mathrm{H}-2^{\prime}\right), 1.94(\mathrm{~d}, 1 \mathrm{H}, \mathrm{H}-5, J=10.4), 3.68-3.74(\mathrm{~m}, 3 \mathrm{H}, 2 \times \mathrm{H}-$ 1, H-4), 3.89 (s, 1H, OH), 4.81 (d, 1H, H-7, J = 17.2), 4.89 (d, 1H, H-7', $J=10.0), 6.10$ - 6.20 (m, 1H, H-6); ${ }^{13} \mathrm{C}$ NMR $(100 \mathrm{MHz})-2.4\left(\mathrm{Si}\left(\mathrm{CH}_{3}\right)_{3}\right), 19.0\left(\mathrm{SiC}\left(\mathrm{CH}_{3}\right)_{3}\right), 23.6\left(\mathrm{CH}_{3}\right), 25.9\left(\mathrm{CH}_{3}\right), 26.8$ $\left(\mathrm{CSi}\left(\mathrm{CH}_{3}\right)_{3}\right), 38.5$ (C-5), 40.1 (C-3), 44.1 (C-2), 61.2 (C-1), 76.5 (C-4), 111.3 (C-7), 127.8 (Ar-C), 129.8 (Ar-C), 132.8 (Ar-C), 132.9 (Ar-C), 135.6 (Ar-C), 137.7 (C-6); MS (CI+) m/z: 451.3 ([M$\left.\left.\mathrm{H}_{2} \mathrm{O}\right] \mathrm{H}^{+}, 1 \%\right), 379.2$ (100\%), 321.2 (66\%), 297.1 (18\%), $243.1(14 \%), 216.1(21 \%), 199.1(43 \%)$, $123.1(82 \%)$; HRMS $m / z$ : [M- $\left.\mathrm{H}_{2} \mathrm{O}\right] \mathrm{H}^{+}$found $451.2835 \mathrm{C}_{28} \mathrm{H}_{43} \mathrm{OSi}_{2}$, requires 451.2852.

$\mathrm{KH}(30 \%$ in mineral oil, $340 \mathrm{mg}, 2.55 \mathrm{mmol}, 3.0$ equiv.) was washed with dry petrol four times and then dried under a flow of argon. A stirred suspension of $\mathrm{KH}$ in THF (12 $\mathrm{mL})$ at $\mathrm{rt}$ was treated slowly with a solution of 1-(tert-butyldiphenylsilyloxy)-3,3-dimethyl-5-trimethylsilylhept-6-en-4-ol (398 mg, $0.85 \mathrm{mmol}$ ). Hydrogen evolution ceased after $5 \mathrm{~min}$ and the mixture was stirred for $1 \mathrm{~h}$. The mixture was poured slowly into sat. aq. $\mathrm{NH}_{4} \mathrm{Cl}(25 \mathrm{~mL})$, the layers were separated and then the aqueous layer extracted with $\mathrm{Et}_{2} \mathrm{O}(20 \mathrm{~mL})$. The combined organic layers were dried $\left(\mathrm{MgSO}_{4}\right)$, filtered and concentrated to give the crude product. Purification by column chromatography (5\% $\mathrm{Et}_{2} \mathrm{O}$ in petrol) gave (4Z)-tert-butyl-(3,3-dimethyl-hepta-4,6-dienyloxy)diphenylsilane (286 mg, 0.76 mmol, $89 \%$ ) as a colorless oil; $R_{\mathrm{f}}=0.8\left(5 \% \mathrm{Et}_{2} \mathrm{O}\right.$ in petrol); IR (film) $3071 \mathrm{w}, 2959 \mathrm{~s}(\mathrm{C}-\mathrm{H}), 2858 \mathrm{~s}$, 1589m, 1472s, 1428s, 1389m, 1363m, 1111s (Si-O), 1047m; ${ }^{1} \mathrm{H}$ NMR (400 MHz) 1.08 (s, 9H, $\left.\mathrm{SiC}\left(\mathrm{CH}_{3}\right)_{3}\right), 1.15\left(\mathrm{~s}, 6 \mathrm{H}, 2 \times \mathrm{CH}_{3}\right), 1.77-1.81(\mathrm{~m}, 2 \mathrm{H}, 2 \times \mathrm{H}-2), 3.73-3.77(\mathrm{~m}, 2 \mathrm{H}, 2 \times \mathrm{H}-1), 5.08-$ $5.15(\mathrm{~m}, 2 \mathrm{H}, 2 \times \mathrm{H}-7), 5.27(\mathrm{~d}, 1 \mathrm{H}, \mathrm{H}-4, J=12.0), 5.81-5.88(\mathrm{~m}, 1 \mathrm{H}, \mathrm{H}-5), 6.75-6.85(\mathrm{~m}, 1 \mathrm{H}, \mathrm{H}-$ 6), 7.38 - 7.47 (m, 6H, Ar-H), 7.69 - 7.72 (m, 4H, Ar-H); ${ }^{13} \mathrm{C}$ NMR (100 MHz) $\left.19.1\left(\mathrm{SiC}_{(\mathrm{CH}}\right)_{3}\right)$, 
$26.9\left(\mathrm{SiC}\left(\mathrm{CH}_{3}\right)_{3}\right), 29.8\left(\mathrm{CH}_{3}\right), 36.0(\mathrm{C}-3), 46.4$ (C-2), $61.3(\mathrm{C}-1), 117.8(\mathrm{C}-7), 127.6(\mathrm{Ar}-\mathrm{C}), 128.4$ (C-5), 129.5 (Ar-C), 133.2 (C-6), 134.0 (Ar-C), 135.6 (Ar-C), 140.6 (C-4); MS (CI+) m/z: 379.2 $\left(\mathrm{MH}^{+}, 100 \%\right), 321.2$ (75\%), 243.1 (14\%), 199.1 (27\%), $123.1(72 \%)$; HRMS m/z: $\mathrm{MH}^{+}$found 379.2459 $\mathrm{C}_{25} \mathrm{H}_{35} \mathrm{OSi}$, requires 379.2457.

TBAF (1.0 M in THF, $49.2 \mathrm{~mL}, 49.2 \mathrm{mmol}, 2.0$ equiv.) was added to a stirred solution of (4Z)-tertbutyl-(3,3-dimethyl-hepta-4,6-dienyloxy)diphenylsilane $(9.30 \mathrm{~g}, 24.6 \mathrm{mmol})$ in THF $(80 \mathrm{~mL})$ at $\mathrm{rt}$ and then stirred for $12 \mathrm{~h}$. The mixture was washed with water $(200 \mathrm{~mL})$, the layers were separated and then the aqueous layer extracted with $\mathrm{Et}_{2} \mathrm{O}(200 \mathrm{~mL})$. The combined organic layers were dried $\left(\mathrm{MgSO}_{4}\right)$, filtered and concentrated to give (4Z)-3,3-dimethylhepta-4,6-dien-1-ol (3.45 g, 24.6 mmol) as a colorless oil, which was sufficiently pure to be used without further purification. DMSO (4.4 mL, $61.5 \mathrm{mmol}, 2.5$ equiv.) was added slowly to a stirred solution of oxalyl chloride (2.6 mL, $27.1 \mathrm{mmol}, 1.1$ equiv. $)$ in $\mathrm{CH}_{2} \mathrm{Cl}_{2}(110 \mathrm{~mL})$ at $-78{ }^{\circ} \mathrm{C}$ and stirred for $5 \mathrm{~min}$. The mixture was treated slowly with a solution of the above alcohol (3.45 g, $24.6 \mathrm{mmol})$ in $\mathrm{CH}_{2} \mathrm{Cl}_{2}(20 \mathrm{~mL})$, stirred for 15 min and then treated slowly with $\mathrm{Et}_{3} \mathrm{~N}(17.1 \mathrm{~mL}, 123 \mathrm{mmol}, 5.0$ equiv.). After $5 \mathrm{~min}$, the mixture was warmed to $\mathrm{rt}$ and stirred for a further $12 \mathrm{~h}$. The reaction mixture was washed with water (100 $\mathrm{mL})$, the layers were separated and then the aqueous layer extracted with $\mathrm{CH}_{2} \mathrm{Cl}_{2}(2 \times 100 \mathrm{~mL})$. The combined organic layers were dried $\left(\mathrm{MgSO}_{4}\right)$, filtered and concentrated to give the crude product. Purification by column chromatography (10\% $\mathrm{Et}_{2} \mathrm{O}$ in petrol) gave (4Z)-3,3-dimethylhepta-4,6dienal $\quad(2.78 \quad \mathrm{~g}, \quad 20.1 \quad \mathrm{mmol}, \quad 82 \% \quad$ from $\quad$ (4Z)-tert-butyl-(3,3-dimethyl-hepta-4,6dienyloxy)diphenylsilane) as a colorless oil, $R_{\mathrm{f}}=0.5\left(10 \% \mathrm{Et}_{2} \mathrm{O}\right.$ in petrol); IR (film) 3089w, 2963s (C-H), 2734m, 1722s (C=O), 1586w, 1470m, 1389w, 1367m, 1254w, 1154w, 1047m; ${ }^{1} \mathrm{H}$ NMR (400 MHz) $1.30\left(\mathrm{~s}, 6 \mathrm{H}, 2 \times \mathrm{CH}_{3}\right), 2.49(\mathrm{~d}, 2 \mathrm{H}, 2 \times \mathrm{H}-2, J=2.0), 5.17-5.21(\mathrm{~m}, 2 \mathrm{H}, 2 \times \mathrm{H}-7), 5.44(\mathrm{~d}$, $1 \mathrm{H}, \mathrm{H}-4, J=12.0), 5.96$ (t, 1H, H-5, $J=12.0), 6.74-6.83(\mathrm{~m}, 1 \mathrm{H}, \mathrm{H}-6), 9.74$ (t, 1H, H-1, $J=4.2)$; ${ }^{3} \mathrm{C}$ NMR (100 MHz) $29.8\left(\mathrm{CH}_{3}\right), 35.8$ (C-3), 56.5 (C-2), 119.4 (C-7), 129.5 (C-5), 132.4 (C-6), $138.2(\mathrm{C}-4), 202.8(\mathrm{C}-1)$; $\mathrm{MS}(\mathrm{CI}+) \mathrm{m} / z: 156.1\left(\mathrm{MNH}_{4}{ }^{+}, 63 \%\right), 148.1(21 \%), 139.1(28 \%), 121.1$ (14\%), 109.1 (16\%), 95.1 (100\%); HRMS m/z: $\mathrm{MNH}_{4}{ }^{+}$found $156.1386 \mathrm{C}_{9} \mathrm{H}_{18} \mathrm{NO}$, requires 156.1388 . 
$n$-BuLi (1.6 $\mathrm{M}$ in hexane, $6.0 \mathrm{~mL}, 9.6 \mathrm{mmol}, 1.1$ equiv.) was added dropwise $(\sim 1 \mathrm{drop} / 2 \mathrm{~s})$ to a stirred solution of (4Z)-3,3-dimethylhepta-4,6-dienal (1.20 g, $8.68 \mathrm{mmol})$ and dibromomethane (0.73 mL, $10.4 \mathrm{mmol}, 1.2$ equiv.) in THF $(35 \mathrm{~mL})$ at $-78{ }^{\circ} \mathrm{C} .{ }^{[6]}$ The resulting mixture was warmed to $\mathrm{rt}$ and stirred for $16 \mathrm{~h}$. The reaction mixture was washed with sat. aq. $\mathrm{NH}_{4} \mathrm{Cl}(40 \mathrm{~mL})$, the layers were separated and then the aqueous layer extracted with $\mathrm{Et}_{2} \mathrm{O}(2 \times 40 \mathrm{~mL})$. The combined organic layers were dried $\left(\mathrm{MgSO}_{4}\right)$, filtered and concentrated to give the crude product. Purification by column chromatography $\left(10 \% \mathrm{Et}_{2} \mathrm{O}\right)$ gave epoxide $\mathbf{4 m}(707 \mathrm{mg}, 4.64 \mathrm{mmol}, 53 \%)$ as a colorless oil, $R_{\mathrm{f}}=0.4\left(10 \% \mathrm{Et}_{2} \mathrm{O}\right.$ in petrol); IR (film) 3088w (epoxide), 3045w, 2962s, 2926s (C-H), 2873m, 1722m, 1470m, 1434w, 1410w, 1366m, 1168m; ${ }^{1} \mathrm{H}$ NMR (400 MHz) 1.24 (s, 3H, CH $), 1.28$ (s, 3H, $\left.\mathrm{CH}_{3}\right), 1.60-1.70(\mathrm{~m}, 2 \mathrm{H}, 2 \times \mathrm{H}-3), 2.44(\mathrm{dd}, 1 \mathrm{H}, \mathrm{H}-1, J=2.8,5.2), 2.74\left(\mathrm{dd}, 1 \mathrm{H}, \mathrm{H}-1^{\prime}, J=4.4\right)$, $2.94-2.98(\mathrm{~m}, 1 \mathrm{H}, \mathrm{H}-2), 5.12-5.18(\mathrm{~m}, 2 \mathrm{H}, 2 \times \mathrm{H}-8), 5.40(\mathrm{~d}, 1 \mathrm{H}, \mathrm{H}-5, J=12.4), 5.94$ (t, 1H, H-6, $J=11.6), 6.78-6.88(\mathrm{~m}, 1 \mathrm{H}, \mathrm{H}-7) ;{ }^{13} \mathrm{C} \mathrm{NMR}(100 \mathrm{MHz}) 29.5\left(\mathrm{CH}_{3}\right), 29.8\left(\mathrm{CH}_{3}\right), 36.8(\mathrm{C}-4), 46.6$ (C-3), 46.9 (C-1), 49.8 (C-2), 118.4 (C-8), 128.9 (C-6), 132.9 (C-7), 139.8 (C-5); MS (CI+) m/z: $170.2\left(\mathrm{MNH}_{4}^{+}, 39 \%\right), 153.1\left(\mathrm{MH}^{+}, 45 \%\right), 135.1(89 \%), 119.1(16 \%), 109.1(20 \%)$; HRMS m/z: $\mathrm{MNH}_{4}{ }^{+}$found $170.1539 \mathrm{C}_{10} \mathrm{H}_{20} \mathrm{NO}$, requires 170.1545 .

endo-4,4-Dimethyl-6-vinylbicyclo[3.1.0]hexan-2-ol 5m

Following the typical cyclopropanation procedure, (5Z)-1,2-epoxy-4,4-dimethylocta-3,5-diene $4 \mathbf{m}$ $(152 \mathrm{mg}, 1.0 \mathrm{mmol})$ was treated with LTMP in $t$-BuOMe for $16 \mathrm{~h}$ to give bicyclic alcohol $\mathbf{5 m}$ (119 $\mathrm{mg}, 0.78 \mathrm{mmol}, 78 \%)$ as a colorless oil, $R_{\mathrm{f}}=0.3\left(40 \% \mathrm{Et}_{2} \mathrm{O}\right.$ in petrol); IR (film) $3355 \mathrm{bs}(\mathrm{O}-\mathrm{H})$, 3081w, 2953s (C-H), 2869m, 1626m, 1455m, 1335m, 1221w, 1145m, 1005s (C=C); ${ }^{1} \mathrm{H}$ NMR $(400$ $\mathrm{MHz}) 1.05\left(\mathrm{~s}, 3 \mathrm{H}, \mathrm{CH}_{3}\right), 1.26\left(\mathrm{~s}, 3 \mathrm{H}, \mathrm{CH}_{3}\right), 1.42-1.46(\mathrm{~m}, 1 \mathrm{H}, \mathrm{H}-6), 1.56-1.77(\mathrm{~m}, 5 \mathrm{H}, \mathrm{H}-1,2 \times$ H-3, H-5, OH), 4.27 (d, 1H, H-2, $J=5.2), 5.05$ (dd, 1H, H-8, $J=1.2,10.0), 5.22$ (dd, 1H, H-8' $J=$ 1.2, 16.8), 5.69 - 5.78 (m, 1H, H-7); ${ }^{13} \mathrm{C}$ NMR (100 MHz) $24.4\left(\mathrm{CH}_{3}\right), 25.8\left(\mathrm{CH}_{3}\right), 31.8(\mathrm{C}-6), 32.9$ (C-1), 37.0 (C-5), 39.8 (C-3), 49.5 (C-4), 74.8 (C-2), 117.0 (C-8), 135.4 (C-7); MS (CI+) m/z: 170.2 $\left(\mathrm{MNH}_{4}{ }^{+}, 2 \%\right), 152.1(8 \%), 135.1(100 \%), 119.1$ (25\%), $107.1(6 \%)$; HRMS $m / z: \mathrm{MNH}_{4}^{+}$found 170.1546 $\mathrm{C}_{10} \mathrm{H}_{20} \mathrm{NO}$, requires 170.1545 . 


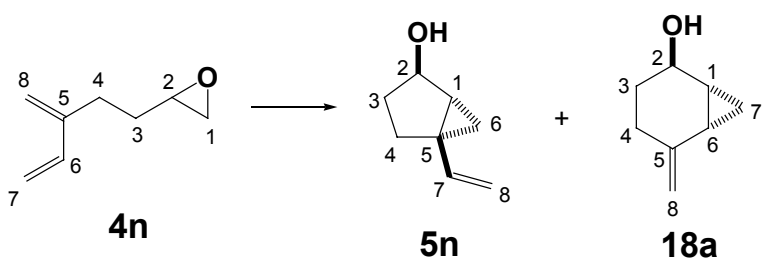

\section{1,2-Epoxy-5-methylene-6-heptene 4n}

$n$-BuLi (1.6 M in hexane, $7.5 \mathrm{~mL}, 12.0 \mathrm{mmol})$ was added slowly to a stirred solution of $t$-BuOK (1.35 g, $12.0 \mathrm{mmol})$ and 2,2,6,6-tetramethylpiperidine (1.69 mL, $10.0 \mathrm{mmol})$ in THF (15 mL) at -78 ${ }^{\circ} \mathrm{C}$. After $45 \mathrm{~min}$, the mixture was treated with isoprene $(2.0 \mathrm{~mL}, 20 \mathrm{mmol})$, and then stirred for a further $45 \mathrm{~min}$ to give a red solution. Epichlorohydrin $(0.47 \mathrm{~mL}, 6.0 \mathrm{mmol})$ was added to the reaction mixture in one portion. After $2 \mathrm{~h}$ at $-78{ }^{\circ} \mathrm{C}$, the mixture was poured into sat. aq. $\mathrm{NH}_{4} \mathrm{Cl}(40$ $\mathrm{mL})$. The layers were separated and the aqueous layer extracted with $\mathrm{Et}_{2} \mathrm{O}(40 \mathrm{~mL})$. The combined organic layers were dried $\left(\mathrm{MgSO}_{4}\right)$, filtered and concentrated to give the crude product. Purification by column chromatography ( $5 \% \mathrm{Et}_{2} \mathrm{O}$ in petrol) gave 1,2-epoxy-5-methylene-6-heptene $4 \mathbf{n}$ (263 mg, $2.12 \mathrm{mmol}, 35 \%)$ as a colorless oil; $R_{\mathrm{f}}=0.4\left(5 \% \mathrm{Et}_{2} \mathrm{O}\right.$ in petrol); IR (film) $3090 \mathrm{w}, 3048 \mathrm{w}$ (epoxide), 2981s, 2924s (C-H), 1596s, 1410w, 1261w; ${ }^{1} \mathrm{H}$ NMR (400 MHz) $1.66-1.83$ (m, 2H, $2 \times \mathrm{H}-3$ ), 2.32 - $2.47(\mathrm{~m}, 2 \mathrm{H}, 2 \times \mathrm{H}-4), 2.50(\mathrm{dd}, 1 \mathrm{H}, \mathrm{H}-1, J=2.8,4.8), 2.77\left(\mathrm{t}, 1 \mathrm{H}, \mathrm{H}-1^{\prime}, J=4.4\right), 2.95-2.99$ (m, $1 \mathrm{H}, \mathrm{H}-2), 5.04(\mathrm{~d}, 2 \mathrm{H}, 2 \times \mathrm{H}-8, J=8.0), 5.09(\mathrm{~d}, 1 \mathrm{H}, \mathrm{H}-7, J=10.8), 5.25\left(\mathrm{~d}, 1 \mathrm{H}, \mathrm{H}-7^{\prime}, J=17.6\right)$, $6.38(\mathrm{dd}, 1 \mathrm{H}, \mathrm{H}-67, J=10.8,17.6) ;{ }^{13} \mathrm{C}$ NMR (100 MHz) $27.5(\mathrm{C}-4), 31.0(\mathrm{C}-3), 47.2(\mathrm{C}-1), 52.0$ (C-2), 113.5 (C-7), 116.1 (C-8), 138.5 (C-6), 145.2 (C-5); MS (CI+) m/z: $142.1\left(\mathrm{MNH}_{4}^{+}, 100 \%\right.$, $125.1\left(\mathrm{MH}^{+}, 41 \%\right), 107.1$ (43\%); HRMS $m / z: \mathrm{MNH}_{4}{ }^{+}$found $142.1233, \mathrm{C}_{8} \mathrm{H}_{16} \mathrm{NO}$ requires 142.1232.

\section{5-Vinylbicyclo[3.1.0]hexan-2-ol 5n and 5-methylenebicyclo[4.1.0]heptan-2-ol 18a}

Following the typical cyclopropanation procedure, 1,2-epoxy-5-methylene-6-heptene $4 \mathbf{n}$ (124 mg, $1.00 \mathrm{mmol}$ ) was treated with LTMP in $t$-BuOMe for $24 \mathrm{~h}$ to give an inseparable mixture of 5 vinylbicyclo[3.1.0]hexan-2-ol 5n and 5-methylenebicyclo[4.1.0]heptan-2-ol 18a (5n:18a = 2.4:1, 60 $\mathrm{mg}, 0.48 \mathrm{mmol}, 48 \%)$ as a colorless oil, $R_{\mathrm{f}}=0.3\left(60 \% \mathrm{Et}_{2} \mathrm{O}\right.$ in petrol); IR (film) $3348 \mathrm{bs}(\mathrm{O}-\mathrm{H})$, 3064w, 2945s, 2873s (C-H), 1642s, 1450m, 1278m; ${ }^{1} \mathrm{H}$ NMR (400 MHz) $0.35-0.39$ (m, 1H, H-7B), $0.57\left(\mathrm{t}, 1 \mathrm{H}, \mathrm{H}-6_{5 \mathrm{u}}, J=4.8\right), 0.74\left(\mathrm{dd}, 1 \mathrm{H}, \mathrm{H}-6^{\prime} \mathrm{A}, J=5.6,8.4\right), 0.85-0.89$ (m, 1H, H-7'B), 1.30 - 
$2.37\left(\mathrm{~m}, 13 \mathrm{H}, \mathrm{H}-1_{5 \mathrm{u}}, 2 \times \mathrm{H}-3_{5 \mathrm{u}}, 2 \times \mathrm{H}-4 \mathrm{~A}, \mathrm{H}-1_{18 \mathrm{a}}, 2 \times \mathrm{H}-3_{18 \mathrm{a}}, 2 \times \mathrm{H}-4_{18 \mathrm{a}}, \mathrm{H}-6_{18 \mathrm{a}}, 2 \times \mathrm{OH}\right), 4.19(\mathrm{~s}$, 1H, H-2B), 4.22 (d, 1H, H-25u $J=2.0), 4.78\left(\mathrm{~d}, 1 \mathrm{H}, \mathrm{H}-8_{18 \mathrm{a}}, J=1.6\right), 4.89$ (s, $\left.1 \mathrm{H}, \mathrm{H}-8^{\prime}{ }_{18 \mathrm{a}}\right), 4.95$ $\left(\mathrm{dd}, 1 \mathrm{H}, \mathrm{H}-8_{5 \mathrm{u}}, J=1.6,10.0\right), 5.06\left(\mathrm{dd}, 1 \mathrm{H}, \mathrm{H}-8^{\prime}{ }_{5 \mathrm{u}}, J=1.2,17.2\right), 5.74\left(\mathrm{dd}, 1 \mathrm{H}, \mathrm{H}-7_{5 \mathrm{u}}, J=10.8\right.$, 17.6); ${ }^{13} \mathrm{C}$ NMR (100 MHz) 10.9 (C-7 $\left.18 \mathrm{a}\right), 15.2\left(\mathrm{C}-6_{5 \mathrm{u}}\right), 17.8,20.3,24.3,25.9,28.6,31.1,33.7,66.3$ (C-2 $18 \mathrm{a}), 74.3\left(\mathrm{C}-2_{5 \mathrm{u}}\right), 142.2\left(\mathrm{C}-7_{5 \mathrm{u}}\right), 145.0\left(\mathrm{C}-5_{18 \mathrm{a}}\right)$; MS (CI+) $m / z: 142.1\left(\mathrm{MNH}_{4}^{+}, 27 \%\right), 124.1$ (27\%), 107.1 (100\%); HRMS m/z: $\mathrm{MNH}_{4}^{+}$found 142.1236, $\mathrm{C}_{8} \mathrm{H}_{16} \mathrm{NO}$ requires 142.1232.

5-Isopropenylbicyclo[3.1.0]hexan-2-ol 5o and 6-methyl-5-methylenebicyclo[4.1.0]heptan-2-ol 18b

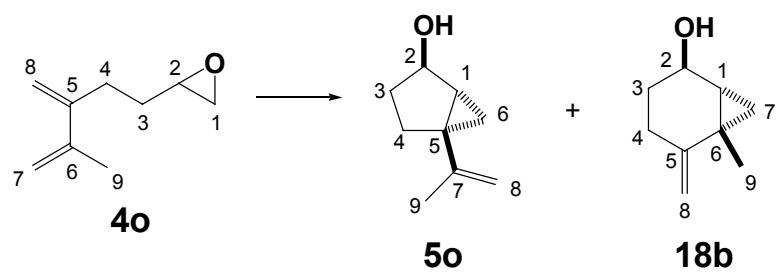

\section{1,2-Epoxy-6-methyl-5-methylene-6-heptene 4o}

$n$-BuLi (1.6 M in hexane, $7.5 \mathrm{~mL}, 12 \mathrm{mmol})$ was added slowly to a stirred solution of $t$-BuOK (1.35 $\mathrm{g}, 12 \mathrm{mmol})$ and 2,2,6,6-tetramethylpiperidine $(1.69 \mathrm{~mL}, 10 \mathrm{mmol})$ in THF at $-78{ }^{\circ} \mathrm{C}$. After $45 \mathrm{~min}$, the mixture was treated with 2,3-dimethyl-1,3-butadiene $(2.3 \mathrm{~mL}, 20 \mathrm{mmol})$, and then stirred for a further $45 \mathrm{~min}$ to give a red solution. Epichlorohydrin $(0.47 \mathrm{~mL}, 6.0 \mathrm{mmol})$ was added rapidly to the red mixture in one portion. After $2 \mathrm{~h}$ at $-78{ }^{\circ} \mathrm{C}$, the reaction mixture was poured into sat. aq. $\mathrm{NH}_{4} \mathrm{Cl}$ $(40 \mathrm{~mL})$, the layers were separated and then the aqueous layer extracted with $\mathrm{Et}_{2} \mathrm{O}(40 \mathrm{~mL})$. The combined organic layers were dried $\left(\mathrm{MgSO}_{4}\right)$, filtered and concentrated to give the crude product. Purification by column chromatography (5\% $\mathrm{Et}_{2} \mathrm{O}$ in petrol) gave 1,2-epoxy-6-methyl-5-methylene6-hepctene 40 (314 mg, $2.27 \mathrm{mmol}, 38 \%)$ as a colorless oil; $R_{\mathrm{f}}=0.4\left(5 \% \mathrm{Et}_{2} \mathrm{O}\right.$ in petrol); IR (film) 3094w (epoxide), 2948s (C-H), 1598s, 1444m, 1410m, 1262w; ${ }^{1} \mathrm{H}$ NMR (400 MHz) 1.67 - 1.80 (m, $2 \mathrm{H}, 2 \times \mathrm{H}-3), 1.92(\mathrm{~s}, 3 \mathrm{H}, 3 \times \mathrm{H}-9), 2.37-2.53(\mathrm{~m}, 3 \mathrm{H}, 2 \times \mathrm{H}-4, \mathrm{H}-1), 2.77\left(\mathrm{t}, 1 \mathrm{H}, \mathrm{H}-1^{\prime}, J=4.8\right)$, $2.93-2.98(\mathrm{~m}, 1 \mathrm{H}, \mathrm{H}-2), 5.00\left(\mathrm{~s}, 2 \mathrm{H}, H_{2} \mathrm{C}=\mathrm{C}\right), 5.09\left(\mathrm{~s}, 1 \mathrm{H}\right.$, one of $\left.H_{2} \mathrm{C}=\mathrm{C}\right), 5.13(\mathrm{~s}, 1 \mathrm{H}$, one of $\left.H_{2} \mathrm{C}=\mathrm{C}\right) ;{ }^{13} \mathrm{C}$ NMR (100 MHz) 21.1 (C-9), 29.9 (C-4), 31.7 (C-3), 47.2 (C-1), 52.1 (C-2), 112.6 $\left(\mathrm{H}_{2} \mathrm{C}=\mathrm{C}\right), 112.8\left(\mathrm{H}_{2} \mathrm{C}=\mathrm{C}\right), 142.3(\mathrm{C}-6), 146.8(\mathrm{C}-5)$; $\mathrm{MS}(\mathrm{CI}+) \mathrm{m} / z: 156.1\left(\mathrm{MNH}_{4}^{+}, 100 \%\right), 139.1$ $\left(\mathrm{MH}^{+}, 25 \%\right), 121.1$ (18\%); HRMS $m / z: \mathrm{MNH}_{4}{ }^{+}$found 156.1385, $\mathrm{C}_{9} \mathrm{H}_{18} \mathrm{NO}$ requires 156.1388. 
Following the typical cyclopropanation procedure, 1,2-epoxy-6-methyl-5-methylene-6-heptene 4o (138 mg, $1.0 \mathrm{mmol}$ ) was treated with LTMP in $t$-BuOMe for $24 \mathrm{~h}$ to give an inseparable mixture of 5-isopropenylbicyclo[3.1.0]hexan-2-ol 50 and 6-methyl-5-methylenebicyclo[4.1.0]heptan-2-ol 18b $(\mathbf{5 o}: \mathbf{1 8 b}=1.1: 1,96 \mathrm{mg}, 0.69 \mathrm{mmol}, 69 \%)$ as a colorless oil, $R_{\mathrm{f}}=0.3\left(50 \% \mathrm{Et}_{2} \mathrm{O}\right.$ in petrol); IR (film) 3346bs (O-H), 3083w, 2947s (C-H), 1634s, 1449s, 1288m, 1171m; ${ }^{1} \mathrm{H}$ NMR (400 MHz) 0.43 (dd, $\left.1 \mathrm{H}, \mathrm{H}-6_{5 \mathrm{v}}, J=4.4,5.6\right), 0.54\left(\mathrm{t}, 1 \mathrm{H}, \mathrm{H}-7_{18 \mathrm{~b}}, J=5.2\right), 0.72\left(\mathrm{dd}, 1 \mathrm{H}, \mathrm{H}-7^{\prime}{ }_{18 \mathrm{~b}}, J=6.8,9.2\right), 0.80-0.83$ $\left(\mathrm{m}, 1 \mathrm{H}, \mathrm{H}-6^{\prime}{ }_{5 \mathrm{v}}\right), 1.11-1.15\left(\mathrm{~m}, 1 \mathrm{H}, \mathrm{H}-1_{18 \mathrm{~b}}\right), 1.25\left(\mathrm{~s}, 3 \mathrm{H}, 3 \times \mathrm{H}-9_{18 \mathrm{~b}}\right), 1.42-1.54(\mathrm{~m}, 3 \mathrm{H}), 1.62-$ $1.66(\mathrm{~m}, 3 \mathrm{H}), 1.68\left(\mathrm{~s}, 3 \mathrm{H}, 3 \times \mathrm{H}-9_{5 \mathrm{v}}\right), 1.77(\mathrm{dd}, 1 \mathrm{H}, J=8.0,12.4), 1.87(\mathrm{bs}, 1 \mathrm{H}, \mathrm{OH}), 1.94-2.02(\mathrm{~m}$, 1H), $2.07-2.15(\mathrm{~m}, 1 \mathrm{H}), 2.28-2.36(\mathrm{~m}, 1 \mathrm{H}), 4.14\left(\mathrm{~s}, 1 \mathrm{H}, \mathrm{H}-2_{18 \mathrm{~b}}\right), 4.23\left(\mathrm{~s}, 1 \mathrm{H}, \mathrm{H}-2_{5 \mathrm{v}}\right), 4.787-4.88$ $\left(\mathrm{m}, 4 \mathrm{H}, 2 \times \mathrm{H}_{2} \mathrm{C}=\mathrm{C}\right) ;{ }^{13} \mathrm{C}$ NMR $(100 \mathrm{MHz}) 13.7\left(\mathrm{C}-6_{5 \mathrm{v}}\right), 18.4\left(\mathrm{C}-7_{18 \mathrm{~b}}\right), 20.3\left(\mathrm{C}-9_{5 \mathrm{v}}\right), 21.2,24.3(\mathrm{C}-$ 918b), 26.2, 27.7, 30.0, 30.1, 31.4, 31.5, 33.9, $67.7\left(\mathrm{C}-2_{18 \mathrm{~b}}\right), 74.6\left(\mathrm{C}-2_{5 \mathrm{v}}\right), 107.7\left(\mathrm{H}_{2} \mathrm{C}=\mathrm{C}\right), 109.3$ $\left(\mathrm{H}_{2} \mathrm{C}=\mathrm{C}\right), 146.8\left(\mathrm{H}_{2} \mathrm{C}=C\right), 149.4\left(\mathrm{H}_{2} \mathrm{C}=C\right) ; \mathrm{MS}(\mathrm{CI}+) m / z: 139.1\left(\mathrm{MH}^{+}, 72 \%\right), 121.1(100 \%) ; \mathrm{HRMS}$ $m / z: \mathrm{MH}^{+}$found $139.1122, \mathrm{C}_{9} \mathrm{H}_{15} \mathrm{O}$ requires 139.1123 .

\section{Synthesis of epoxides 20 and 22}

\section{1,2-Epoxy-3,3-diethyl-4-pentene 20}
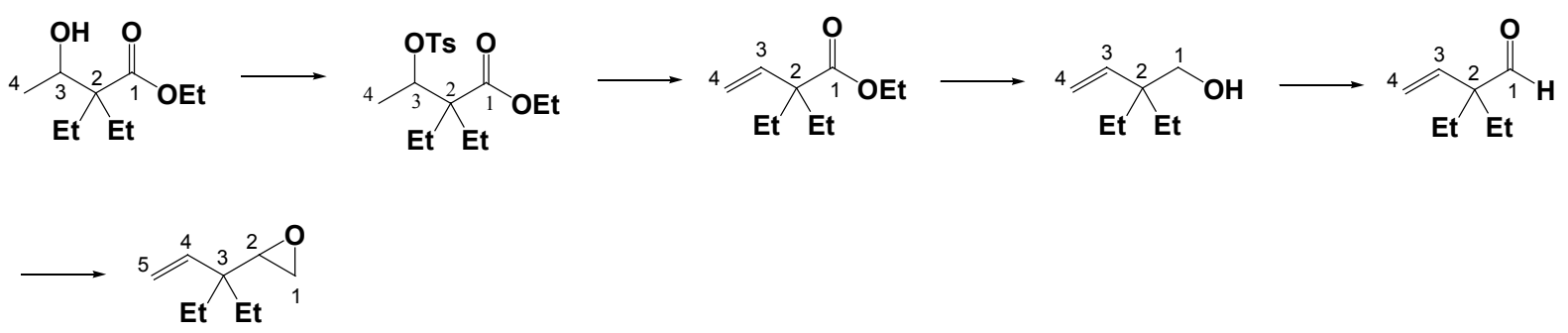

20

n-BuLi (1.6 $\mathrm{M}$ in hexane, $4.8 \mathrm{~mL}, 7.6 \mathrm{mmol}, 1.1$ equiv.) was added to a stirred solution of diisopropylamine $(7.3 \mathrm{~mL}, 7.62 \mathrm{mmol}, 1.1$ equiv. $)$ in THF $(20 \mathrm{~mL})$ at $-78{ }^{\circ} \mathrm{C}$. The LDA solution was stirred at $\mathrm{rt}$ for $15 \mathrm{~min}$, cooled to $-78{ }^{\circ} \mathrm{C}$ and then treated slowly with a solution of ethyl 2ethylbut-1-anoate $(1.0 \mathrm{~g}, 6.93 \mathrm{mmol})$ in THF $(5 \mathrm{~mL})$. The resulting mixture was stirred for $1 \mathrm{~h}$ and treated with acetaldehyde $(0.5 \mathrm{~mL}, 9.01 \mathrm{mmol}, 1.3$ equiv. $)$. After $30 \mathrm{~min}$, the reaction mixture was quenched by slow addition of sat. aq. $\mathrm{NH}_{4} \mathrm{Cl}(5 \mathrm{~mL})$ and then warmed to rt. The solution was washed with sat. aq. $\mathrm{NH}_{4} \mathrm{Cl}(30 \mathrm{~mL})$, the layers were separated and then the aqueous layer extracted 
with $\mathrm{Et}_{2} \mathrm{O}(30 \mathrm{~mL})$. The combined organic layers were dried $\left(\mathrm{MgSO}_{4}\right)$, filtered and concentrated to give the crude product. Purification by column chromatography $\left(30 \% \mathrm{Et}_{2} \mathrm{O}\right.$ in petrol) gave ethyl 2,2-diethyl-3-hydroxybutyrate $(1.03 \mathrm{~g}, 5.47 \mathrm{mmol}, 79 \%)$ as a colorless oil, $R_{\mathrm{f}}=0.3\left(30 \% \mathrm{Et}_{2} \mathrm{O}\right.$ in petrol); IR (film) 3498bs (O-H), 2976s, 2944s, 2884s (C-H), 1723s (C=O), 1462s, 1383s, 1341w, 1294m, 1232s, 1141s, 1098s, 1025s; ${ }^{1} \mathrm{H}$ NMR (500 MHz) 0.81 (t, 3H, $\left.\alpha-\mathrm{CH}_{2} \mathrm{CH}_{3}, J=7.5\right), 0.87$ (t, $\left.3 \mathrm{H}, \alpha-\mathrm{CH}_{2} \mathrm{CH}_{3}, J=7.5\right), 1.13(\mathrm{~d}, 3 \mathrm{H}, 3 \times \mathrm{H}-4, J=6.5), 1.27\left(\mathrm{t}, 3 \mathrm{H}, \mathrm{OCH}_{2} \mathrm{CH}_{3}, J=7.5\right), 1.46-1.82$ $\left(\mathrm{m}, 4 \mathrm{H}, 2 \times \alpha-\mathrm{CH}_{2} \mathrm{CH}_{3}\right), 3.03(\mathrm{~d}, 1 \mathrm{H}, \mathrm{OH}, J=7.0), 3.89-3.94(\mathrm{~m}, 1 \mathrm{H}, \mathrm{H}-3), 4.17(\mathrm{q}, 2 \mathrm{H}$, $\left.\mathrm{OCH}_{2} \mathrm{CH}_{3}, J=7.0\right) ;{ }^{13} \mathrm{C} \mathrm{NMR}(125 \mathrm{MHz}) 8.6\left(\alpha-\mathrm{CH}_{2} \mathrm{CH}_{3}\right), 8.7\left(\alpha-\mathrm{CH}_{2} \mathrm{CH}_{3}\right), 14.2\left(\mathrm{OCH}_{2} \mathrm{CH}_{3}\right), 17.9$ (C-4), $23.8\left(\alpha-\mathrm{CH}_{2} \mathrm{CH}_{3}\right), 25.5\left(\alpha-\mathrm{CH}_{2} \mathrm{CH}_{3}\right), 53.7$ (C-2), $60.4\left(\mathrm{OCH}_{2} \mathrm{CH}_{3}\right), 69.8(\mathrm{C}-3), 176.9(\mathrm{C}-1)$; MS (CI+) m/z: $206.2\left(\mathrm{MNH}_{4}^{+}, 2 \%\right), 189.1\left(\mathrm{MH}^{+}, 100 \%\right), 171.1(80 \%), 160.1(18 \%), 144.1(91 \%)$, $129.1(25 \%), 115.1$ (13\%); HRMS $m / z: \mathrm{MNH}_{4}^{+}$found 206.1758, $\mathrm{C}_{10} \mathrm{H}_{24} \mathrm{NO}_{3}$ requires 206.1756.

p-Toluenesulfonyl chloride (11.4 g, $59.8 \mathrm{mmol}, 2.0$ equiv.) was added to a stirred solution of ethyl 2,2-diethyl-3-hydroxybutyrate $(5.63 \mathrm{~g}, 29.9 \mathrm{mmol})$ in dry pyridine $(20 \mathrm{~mL})$ at $0{ }^{\circ} \mathrm{C}$. The resulting mixture was stirred at $\mathrm{rt}$ for $48 \mathrm{~h}$. The mixture was diluted with EtOAc $(150 \mathrm{~mL})$, washed with aq. $\mathrm{HCl}(1 \mathrm{M})$ until $\mathrm{pH}$ of the aqueous layer $<3$ and the layers were separated. The organic layer was dried $\left(\mathrm{MgSO}_{4}\right)$, filtered and concentrated to give the crude product. Purification by column chromatography (10\% EtOAc in petrol) gave ethyl 2,2-diethyl-3-(toluene-4-sulfonyloxy)butyrate $(8.01 \mathrm{~g}, 23.4 \mathrm{mmol}, 78 \%)$ as a colorless oil; $R_{\mathrm{f}}=0.3(10 \%$ EtOAc in petrol); IR (film) $1278 \mathrm{~s}, 2885 \mathrm{~s}$ $(\mathrm{C}-\mathrm{H}), 1732 \mathrm{~s}(\mathrm{C}=\mathrm{O}), 1599 \mathrm{~m}, 1496 \mathrm{w}, 1454 \mathrm{~m}, 1363 \mathrm{~s}, 1306 \mathrm{w}, 1235 \mathrm{~s}, 1189 \mathrm{~s}, 1177 \mathrm{~s}, 1144 \mathrm{~m}, 1098 \mathrm{~m}$, 1076w, 1020m; ${ }^{1} \mathrm{H}$ NMR (400 MHz) $0.81\left(\mathrm{t}, 3 \mathrm{H}, \alpha-\mathrm{CH}_{2} \mathrm{CH}_{3}, J=7.0\right), 0.83\left(\mathrm{t}, 3 \mathrm{H}, \alpha-\mathrm{CH}_{2} \mathrm{CH}_{3}, J=\right.$ 7.0), $1.22\left(\mathrm{t}, 3 \mathrm{H}, \mathrm{OCH}_{2} \mathrm{CH}_{3}, J=7.5\right), 1.31(\mathrm{~d}, 3 \mathrm{H}, 3 \times \mathrm{H}-4, J=6.5), 1.54-1.77(\mathrm{~m}, 4 \mathrm{H}, 2 \times \alpha-$ $\left.\mathrm{CH}_{2} \mathrm{CH}_{3}\right), 2.44\left(\mathrm{~s}, 3 \mathrm{H}, \mathrm{ArCH}_{3}\right), 4.06-4.13\left(\mathrm{~m}, 2 \mathrm{H}, \mathrm{OCH}_{2} \mathrm{CH}_{3}\right), 4.99-5.03(\mathrm{~m}, 1 \mathrm{H}, \mathrm{H}-3), 7.32$ (d, $2 \mathrm{H}, 2 \times \mathrm{Ar}-\mathrm{H}, J=8.5), 7.78(\mathrm{~d}, 2 \mathrm{H}, 2 \times \mathrm{Ar}-\mathrm{H}, J=8.0) ;{ }^{1} \mathrm{H}$ NMR $(100 \mathrm{MHz}) 8.5\left(\alpha-\mathrm{CH}_{2} \mathrm{CH}_{3}\right), 8.7$ $\left(\alpha-\mathrm{CH}_{2} \mathrm{CH}_{3}\right), 14.1\left(\mathrm{OCH}_{2} \mathrm{CH}_{3}\right), 16.8(\mathrm{C}-4), 21.6\left(\mathrm{ArCH}_{3}\right), 23.9\left(\alpha-\mathrm{CH}_{2} \mathrm{CH}_{3}\right), 24.4\left(\alpha-\mathrm{CH}_{2} \mathrm{CH}_{3}\right), 53.6$ (C-2), $60.6\left(\mathrm{OCH}_{2} \mathrm{CH}_{3}\right), 82.3$ (C-3), 127.5 (Ar-C), 129.6 (Ar-C), 135.0 (Ar-C), 144.3 (Ar-C), 173.5 (C-1); MS (CI+) m/z: $360.2\left(\mathrm{MNH}_{4}^{+}, 5 \%\right), 343.2\left(\mathrm{MH}^{+}, 2 \%\right), 298.1(2 \%), 188.16(5 \%), 171.1$ 
(100\%), 148.1 (17\%), 141.1 (29\%), 124.1 (9\%), 113.1 (5\%); HRMS $m / z: \mathrm{MNH}_{4}{ }^{+}$found 360.1838, $\mathrm{C}_{17} \mathrm{H}_{30} \mathrm{NO}_{5}$ requires 360.1845 .

A mixture of ethyl 2,2-diethyl-3-(toluene-4-sulfonyloxy)butyrate (8.01 g, $23.4 \mathrm{mmol})$ in DBU (9.5 $\mathrm{mL}$ ) was heated under reflux at $140{ }^{\circ} \mathrm{C}$ for $3 \mathrm{~h}$. The resulting mixture was cooled to rt, diluted with $\mathrm{Et}_{2} \mathrm{O}(150 \mathrm{~mL})$, washed with aq. $\mathrm{HCl}(1 \mathrm{M}, 150 \mathrm{~mL})$ until the aqueous layer reached $\mathrm{pH}<3$ and the layers were separated. The organic layer was dried $\left(\mathrm{MgSO}_{4}\right)$, filtered and concentrated to give ethyl 2,2-diethylbut-3-enoate (3.67 mg, $21.6 \mathrm{mmol}, 92 \%)$ as a light yellow oil, which was sufficiently pure to be used without further purification; IR (film) 2973s, 2941s, 2882s (C-H), 1731s (C=O), $1639 \mathrm{w}, 1462 \mathrm{~s}, 1413 \mathrm{w}, 1382 \mathrm{~m}, 1369 \mathrm{~m}, 1345 \mathrm{w}, 1296 \mathrm{w}, 1228 \mathrm{~s}, 1133 \mathrm{~s}, 1028 \mathrm{~m}, 1002 \mathrm{w} ;{ }^{1} \mathrm{H}$ NMR $(500$ MHz) $0.82\left(\mathrm{t}, 6 \mathrm{H}, 2 \times \alpha-\mathrm{CH}_{2} \mathrm{CH}_{3}, J=7.5\right), 1.26\left(\mathrm{t}, 3 \mathrm{H}, \mathrm{OCH}_{2} \mathrm{CH}_{3}, J=7.0\right), 1.73(\mathrm{q}, 4 \mathrm{H}, 2 \times \alpha-$ $\left.\mathrm{CH}_{2} \mathrm{CH}_{3}, J=7.0\right), 4.16$ (q, 2H, $\left.\mathrm{OCH}_{2} \mathrm{CH}_{3}, J=7.0\right), 5.08$ (d, 1H, H-4, $\left.J=17.5\right), 5.19$ (d, $1 \mathrm{H}, \mathrm{H}-4^{\prime}, J$ $=12.0), 5.97(\mathrm{dd}, 1 \mathrm{H}, \mathrm{H}-3, J=11.0,18.0) ;{ }^{13} \mathrm{C} \mathrm{NMR}(125 \mathrm{MHz}) 8.7\left(\alpha-\mathrm{CH}_{2} \mathrm{CH}_{3}\right), 14.2$ $\left(\mathrm{OCH}_{2} \mathrm{CH}_{3}\right), 28.3\left(\alpha-\mathrm{CH}_{2} \mathrm{CH}_{3}\right), 53.0(\mathrm{C}-2), 60.4\left(\mathrm{OCH}_{2} \mathrm{CH}_{3}\right), 114.4(\mathrm{C}-4), 139.8(\mathrm{C}-3), 175.4(\mathrm{C}-1)$; MS (CI+) m/z: $188.2\left(\mathrm{MNH}_{4}^{+}, 9 \%\right), 171.1\left(\mathrm{MH}^{+}, 100 \%\right), 155.1(3 \%), 148.1(11 \%), 141.1(42 \%)$, $124.1(15 \%), 113.1$ (4\%); HRMS $m / z: \mathrm{MNH}_{4}^{+}$found 188.1650, $\mathrm{C}_{10} \mathrm{H}_{22} \mathrm{NO}_{2}$ requires 188.1651.

$\mathrm{LiAlH}_{4}(2.3 \mathrm{M}$ in THF, $71.7 \mathrm{~mL}, 165 \mathrm{mmol}, 2.2$ equiv.) was added slowly to a stirred solution of ethyl 2,2-diethylbut-3-enoate $(12.8 \mathrm{~g}, 75.2 \mathrm{mmol})$ in THF $(350 \mathrm{~mL})$ at $0{ }^{\circ} \mathrm{C}$. After $4 \mathrm{~h}$, the mixture was cooled to $-78{ }^{\circ} \mathrm{C}$ and quenched by sequential addition of water $(6.3 \mathrm{~mL})$, aq. $\mathrm{NaOH}(15 \mathrm{w} \%, 6.3$ $\mathrm{mL})$ and water $(18.9 \mathrm{~mL})$. The precipitates formed were filtered and washed through with $\mathrm{Et}_{2} \mathrm{O}(200$ $\mathrm{mL})$. The filtrate was dried $\left(\mathrm{MgSO}_{4}\right)$, filtered and concentrated to give 2,2-diethylbut-3-en-1-ol $(8.17 \mathrm{~g}, 63.7 \mathrm{mmol}, 85 \%)$ as a colorless oil, which was sufficiently pure to be used without further purification; $R_{\mathrm{f}}=0.3\left(30 \% \mathrm{Et}_{2} \mathrm{O}\right.$ in petrol); IR (film) $3370 \mathrm{bs}(\mathrm{O}-\mathrm{H}), 3082 \mathrm{~m}(\mathrm{C}=\mathrm{C}), 2967 \mathrm{~s}, 2880 \mathrm{~s}(\mathrm{C}-$ $\mathrm{H}), 1638 \mathrm{~m}, 1462 \mathrm{~s}, 1416 \mathrm{~m}, 1380 \mathrm{~m}, 1244 \mathrm{w}, 1165 \mathrm{w}, 1078 \mathrm{~m}, 1030 \mathrm{~s}, 1003 \mathrm{~m} ;{ }^{1} \mathrm{H}$ NMR (400 MHz) $0.81\left(\mathrm{t}, 6 \mathrm{H}, 2 \times \alpha-\mathrm{CH}_{2} \mathrm{CH}_{3}, J=7.6\right), 1.29-1.48\left(\mathrm{~m}, 4 \mathrm{H}, 2 \times \alpha-\mathrm{CH}_{2} \mathrm{CH}_{3}\right), 3.42(\mathrm{~s}, 1 \mathrm{H}, \mathrm{OH}), 5.02(\mathrm{dd}$, $1 \mathrm{H}, \mathrm{H}-4, J=1.2,17.6), 5.21\left(\mathrm{dd}, 1 \mathrm{H}, \mathrm{H}-4{ }^{\prime}, J=1.2,6.7\right), 5.63(\mathrm{dd}, 1 \mathrm{H}, \mathrm{H}-3, J=10.8,17.6) ;{ }^{13} \mathrm{C}$ NMR (100 MHz) $7.5\left(\alpha-\mathrm{CH}_{2} \mathrm{CH}_{3}\right), 24.7\left(\alpha-\mathrm{CH}_{2} \mathrm{CH}_{3}\right), 44.8(\mathrm{C}-2), 66.1(\mathrm{C}-1), 115.0(\mathrm{C}-4), 143.8(\mathrm{C}-$ 
3); $\mathrm{MS}(\mathrm{CI}+) \mathrm{m} / z: 148.1$ (34\%), $146.2\left(\mathrm{MNH}_{4}^{+}, 41 \%\right), 131.1(6 \%), 110.1(38 \%)$; HRMS m/z: $\mathrm{MNH}_{4}^{+}$found 146.1545, $\mathrm{C}_{8} \mathrm{H}_{20} \mathrm{NO}$ requires 146.1544.

TPAP (137 mg, $0.39 \mathrm{mmol}, 5 \mathrm{~mol} \%$ ) was added to a stirred solution of 2,2-diethylbut-3-en-1-ol (1.00 g, $7.80 \mathrm{mmol})$, 4-methylmorpholine $N$-oxide (15.62 mmol, $1.83 \mathrm{~g}, 2.0$ equiv.) and powdered 3 $\AA$ molecular sieves $(2.0 \mathrm{~g})$ in $\mathrm{CH}_{2} \mathrm{Cl}_{2}(50 \mathrm{~mL})$ at $\mathrm{rt}$. After $4 \mathrm{~h}$, the solution was filtered through a pad of silica $(11 \mathrm{~cm} \times 3 \mathrm{~cm})$ topped with a small amount of celite, and then eluted through with $\mathrm{CH}_{2} \mathrm{Cl}_{2}(100 \mathrm{~mL})$. The filtrate was concentrated to give 2,2-diethylbut-3-enal (756 mg, $6.00 \mathrm{mmol}$, $77 \%$ ) as a colorless oil, which was sufficiently pure to be used without further purification; $R_{\mathrm{f}}=0.6$ (10\% $\mathrm{Et}_{2} \mathrm{O}$ in petrol); IR (film) 3085w (C=C), 2970s, 2940s, 2882s $(\mathrm{C}-\mathrm{H}), 1727 \mathrm{~s}(\mathrm{C}=\mathrm{O}), 1632 \mathrm{~m}$, 1459m, 1414w, 1383m, 1001m; ${ }^{1} \mathrm{H}$ NMR (400 MHz) 0.82 (t, 6H, $\left.2 \times \alpha-\mathrm{CH}_{2} \mathrm{CH}_{3}, J=7.6\right), 1.60-$ $1.75\left(\mathrm{~m}, 4 \mathrm{H}, 2 \times \alpha-\mathrm{CH}_{2} \mathrm{CH}_{3}\right), 5.13(\mathrm{~d}, 1 \mathrm{H}, \mathrm{H}-4, J=18.0), 5.34\left(\mathrm{~d}, 1 \mathrm{H}, \mathrm{H}-4^{\prime}, J=10.8\right), 5.71(\mathrm{dd}, 1 \mathrm{H}$, $\mathrm{H}-3, J=10.8,17.6), 9.38(\mathrm{~s}, 1 \mathrm{H}, \mathrm{CHO}) ;{ }^{13} \mathrm{C} \mathrm{NMR}(100 \mathrm{MHz}) 8.0\left(\alpha-\mathrm{CH}_{2} \mathrm{CH}_{3}\right), 24.6\left(\alpha-\mathrm{CH}_{2} \mathrm{CH}_{3}\right)$, 56.5 (C-2), 117.5 (C-4), 137.6 (C-3), 203.7 (C-1); MS (CI+) m/z: $144.1\left(\mathrm{MNH}_{4}^{+}, 100 \%\right), 141.1$ (3\%), $131.1(9 \%), 126.1\left(\mathrm{M}^{+}, 27 \%\right)$; HRMS $m / z: \mathrm{MNH}_{4}{ }^{+}$found $144.1382, \mathrm{C}_{8} \mathrm{H}_{18} \mathrm{NO}$ requires 144.1388.

$n$-BuLi (1.6 M in hexane, $3.8 \mathrm{~mL}, 6.1 \mathrm{mmol}, 1.1$ equiv.) was added dropwise ( $1 \mathrm{drop} / 2 \mathrm{~s})$ to a stirred solution of 2,2-diethylbut-3-enal $(700 \mathrm{mg}, 5.55 \mathrm{mmol})$ and dibromomethane $(0.47 \mathrm{~mL}, 6.66$ mmol, 1.2 equiv. $)$ in $\mathrm{THF}(20 \mathrm{~mL})$ at $-78{ }^{\circ} \mathrm{C}$. The resulting mixture was warmed to rt and stirred for 20 h. The reaction mixture was washed with sat. aq. $\mathrm{NH}_{4} \mathrm{Cl}(50 \mathrm{~mL})$, the layers were separated and then the aqueous layer extracted with $\mathrm{Et}_{2} \mathrm{O}(2 \times 40 \mathrm{~mL})$. The combined organic layers were dried $\left(\mathrm{MgSO}_{4}\right)$, filtered and concentrated to give the crude product. Purification by column chromatography (10\% $\left.\mathrm{Et}_{2} \mathrm{O}\right)$ gave 1,2-epoxy-3,3-diethyl-4-pentene $\mathbf{2 0}(598 \mathrm{mg}, 4.26 \mathrm{mmol}, 77 \%)$ as a colorless oil, $R_{\mathrm{f}}=0.6\left(10 \% \mathrm{Et}_{2} \mathrm{O}\right.$ in petrol); IR (film) 3084w (C=C), 3051w (epoxide), 2969s, 2927s, 2882s (C-H), 1636m, 1462s, 1414m, 1383m, 1261w, 1082w, 1014m; ${ }^{1} \mathrm{H}$ NMR (400 MHz) $0.85\left(\mathrm{t}, 3 \mathrm{H}, \alpha-\mathrm{CH}_{2} \mathrm{CH}_{3}, J=7.2\right), 0.87\left(\mathrm{t}, 3 \mathrm{H}, \alpha-\mathrm{CH}_{2} \mathrm{CH}_{3}, J=7.2\right), 1.40-1.58(\mathrm{~m}, 4 \mathrm{H}, 2 \times \alpha-$ $\mathrm{CH}_{2} \mathrm{CH}_{3}$ ), 2.57 (dd, 1H, H-1, $\left.J=3.2,4.8\right), 2.64$ (dd, 1H, H-1', $\left.J=4.4,4.8\right), 2.85$ (dd, 1H, H-2, $J=$ 
3.2, 3.6), $5.03(\mathrm{dd}, 1 \mathrm{H}, \mathrm{H}-5, J=1.2,18.0), 5.14\left(\mathrm{~d}, 1 \mathrm{H}, \mathrm{H}-5^{\prime}, J=11.2\right), 5.56(\mathrm{dd}, 1 \mathrm{H}, \mathrm{H}-4, J=11.2$, 18.0); ${ }^{13} \mathrm{C}$ NMR (100 MHz) 7.9, $8.0\left(\alpha-\mathrm{CH}_{2} \mathrm{CH}_{3}\right), 25.5\left(\alpha-\mathrm{CH}_{2} \mathrm{CH}_{3}\right), 27.4\left(\alpha-\mathrm{CH}_{2} \mathrm{CH}_{3}\right), 42.2(\mathrm{C}-3)$, 43.5 (C-1), 57.5 (C-2), 114.9 (C-5), 140.0 (C-4); MS (CI+) m/z: $158.2\left(\mathrm{MNH}_{4}^{+}, 6 \%\right), 141.1\left(\mathrm{MH}^{+}\right.$, 8\%), 123.1 (56\%), 111.1 (100\%); HRMS $m / z: \mathrm{MNH}_{4}{ }^{+}$found 158.1537, $\mathrm{C}_{9} \mathrm{H}_{20} \mathrm{NO}$ requires 158.1545.

\section{Dimerization of epoxide 20}

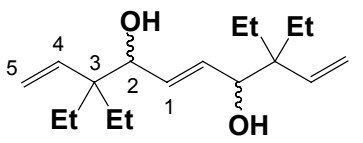

Following the typical procedure, 1,2-epoxy-3,3-diethyl-4-pentene 20 (140 $\mathrm{mg}, 1.00 \mathrm{mmol}$ ) was treated with LTMP in $t$-BuOMe for $20 \mathrm{~h}$ to give the corresponding dimer A (16 $\mathrm{mg}, 11 \%)$ as a pale brown solid, $R_{\mathrm{f}}=0.6\left(\mathrm{Et}_{2} \mathrm{O}\right)$ and dimer $\mathrm{B}(78 \mathrm{mg}, 56 \%)$ as a white solid, $R_{\mathrm{f}}=0.5$ $\left(\mathrm{Et}_{2} \mathrm{O}\right)$

\section{Characterisation data for dimer A:}

IR (film) 3368bs (O-H), 3079s (C=C), 2965s, 2930s, 2881s (C-H), 1636w, 1477s, 1428m, 1383m; ${ }^{1} \mathrm{H}$ NMR (400 MHz) $0.77-0.90\left(\mathrm{~m}, 12 \mathrm{H}, 4 \times \mathrm{CH}_{2} \mathrm{CH}_{3}\right), 1.31-1.69\left(\mathrm{~m}, 8 \mathrm{H}, 4 \times \mathrm{CH}_{2} \mathrm{CH}_{3}\right), 4.36-$ $4.40\left(\mathrm{~m}, 2 \mathrm{H}, \mathrm{H}-2, \mathrm{H}-2^{\prime}\right), 5.06(\mathrm{dd}, 2 \mathrm{H}, 2 \times \mathrm{H}-5, J=1.6,17.6), 5.26\left(\mathrm{dd}, 2 \mathrm{H}, 2 \times \mathrm{H}-5^{\prime}, J=1.6,11.2\right)$, 5.73 - 5.82 (m, 4H, H-1, H-1', H-4, H-4 $\left.{ }^{\prime}\right) ;{ }^{13} \mathrm{C}$ NMR (100 MHz) 7.6, $7.7\left(\mathrm{CHCH}_{3}\right), 23.6,23.9$ $\left(\mathrm{CH}_{2} \mathrm{CH}_{3}\right), 46.1\left(\mathrm{C}-3,3^{\prime}\right), 70.2\left(\mathrm{C}-2,2^{\prime}\right), 116.0\left(\mathrm{C}-5,5^{\prime}\right), 133.2\left(\mathrm{C}-1,1^{\prime}\right), 141.5\left(\mathrm{C}-4,4^{\prime}\right)$.

\section{Characterisation data for dimer $B$ :}

IR (film) 3365bs (O-H), 3079s (C=C), 2965s, 2932s, 2887s (C-H), 1633w, 1479s, 1431m, 1397m; ${ }^{1} \mathrm{H}$ NMR (400 MHz) $0.75-0.84\left(\mathrm{~m}, 12 \mathrm{H}, 4 \times \mathrm{CH}_{2} \mathrm{CH}_{3}\right), 1.31-1.67\left(\mathrm{~m}, 8 \mathrm{H}, 4 \times \mathrm{CH}_{2} \mathrm{CH}_{3}\right), 3.97-$ $3.98\left(\mathrm{~m}, 2 \mathrm{H}, \mathrm{H}-2, \mathrm{H}-2^{\prime}\right), 5.04(\mathrm{~d}, 2 \mathrm{H}, 2 \times \mathrm{H}-5, J=18.0), 5.25\left(\mathrm{~d}, 2 \mathrm{H}, 2 \times \mathrm{H}-5^{\prime}, J=10.8\right), 5.69-5.76$ (m, 4H, H-1, H-1', H-4, H-4'); ${ }^{13} \mathrm{C}$ NMR (100 MHz) 7.6, $7.7\left(\mathrm{CH}_{2} \mathrm{CH}_{3}\right), 23.5,24.5\left(\mathrm{CH}_{2} \mathrm{CH}_{3}\right), 46.8$ (C-3, 3'), 75.9 (C-2, C-2'), $115.9\left(\mathrm{C}-5, \mathrm{C}-5^{\prime}\right), 132.1$ (C-1, C-1' ), 141.8 (C-4, C-4'). 


\section{1,2-Epoxy-5-methylhexane 22 $2^{[13]}$}

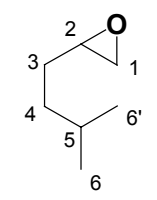

$m$-Chloroperbenzoic acid (77\%, $2.73 \mathrm{~g}, 12.2 \mathrm{mmol}, 1.2$ equiv.) was added to a stirred solution of 5-methylhex-1-ene (1.00 g, $10.2 \mathrm{mmol})$ in $\mathrm{CH}_{2} \mathrm{Cl}_{2}(50 \mathrm{~mL})$ at $\mathrm{rt}$ After $12 \mathrm{~h}$, the solution was successively washed with sat. aq $\mathrm{NaHCO}_{3}(30 \mathrm{~mL})$ and sat. aq. $\mathrm{Na}_{2} \mathrm{SO}_{3}(30 \mathrm{~mL})$. The organic layer was dried $\left(\mathrm{MgSO}_{4}\right)$, filtered and concentrated to give the crude product. Purification by column chromatography $\left(5 \% \mathrm{Et}_{2} \mathrm{O}\right.$ in petrol) gave 1,2-epoxy-5-methylhexane 22 (862 mg, 7.55 mmol, 74\%) as a colorless oil; $R_{\mathrm{f}}=0.4\left(5 \% \mathrm{Et}_{2} \mathrm{O}\right.$ in petrol); IR (film) $2956 \mathrm{~s}(\mathrm{C}-\mathrm{H}), 2870 \mathrm{~m}, 1771 \mathrm{~m}$, $1725 \mathrm{~s}, 1575 \mathrm{w}, 1469 \mathrm{~m}, 1427 \mathrm{~m}, 1385 \mathrm{~m}, 1283 \mathrm{~m}, 1255 \mathrm{~s}, 1217 \mathrm{~s} ;{ }^{1} \mathrm{H}$ NMR (400 MHz) $0.88(\mathrm{~s}, 3 \mathrm{H}$, $\left.\mathrm{CH}_{3}\right), 090\left(\mathrm{~s}, 3 \mathrm{H}, \mathrm{CH}_{3}\right), 1.25-1.43(\mathrm{~m}, 2 \mathrm{H}, 2 \times \mathrm{H}-4), 1.50-1.63(\mathrm{~m}, 3 \mathrm{H}, 2 \times \mathrm{H}-3, \mathrm{H}-5), 2.46(\mathrm{dd}$, $1 \mathrm{H}, \mathrm{H}-1, J=2.4,4.8), 2.74\left(\mathrm{t}, 1 \mathrm{H}, \mathrm{H}-1^{\prime}, J=4.8\right), 2.87-2.91(\mathrm{~m}, 1 \mathrm{H}, \mathrm{H}-2) ;{ }^{13} \mathrm{C}$ NMR $(100 \mathrm{MHz})$ 22.4 (C-6), 27.8 (C-5), 30.3 (C-3), 34.8 (C-4), 47.1 (C-1), 52.5 (C-2).

\section{Synthesis of cyclopropanes $23,24 a-g, 25,26$ and 27}

tert-Butyldimethyl-\{6-(tributylstannyl)bicyclo[3.1.0]hex-2-yloxy\}silane 23

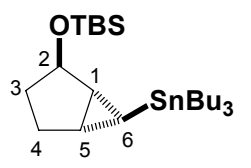

tert-Butyldimethylsilyl chloride (271 mg, $1.80 \mathrm{mmol}, 1.5$ equiv.) was added to a stirred solution of exo-6-tributylstannylbicyclo[3.1.0]hexan-2-ol 5e (464 $\mathrm{mg}, 1.20 \mathrm{mmol}$ ) and imidazole (163 mg, $2.40 \mathrm{mmol}, 2.0$ equiv.) in $\mathrm{CH}_{2} \mathrm{Cl}_{2}(10 \mathrm{~mL})$ at $\mathrm{rt}$. The resulting mixture was stirred for $3 \mathrm{~h}$, and then dry-loaded onto a small amount of silica. Purification by column chromatography $\left(2 \% \mathrm{Et}_{2} \mathrm{O}\right.$ in petrol) gave silylated bicyclic alcohol $23(512 \mathrm{mg}, 1.02 \mathrm{mmol}, 85 \%)$ as a colorless oil, $R_{\mathrm{f}}=0.5\left(2 \% \mathrm{Et}_{2} \mathrm{O}\right.$ in petrol); IR (film) $2957 \mathrm{~s}, 2928 \mathrm{~s}, 2857 \mathrm{~s}(\mathrm{C}-\mathrm{H}), 1463 \mathrm{~m}, 1376 \mathrm{w}$, 1254s, 1183m, 1094m, 1072s (Si-O), 1026s; ${ }^{1} \mathrm{H}$ NMR (400 MHz) -0.61 - -0.54 (m, 1H, H-6), 0.08 (s, 3H, $\left.\mathrm{SiCH}_{3}\right), 0.09$ (s, 3H, $\left.\mathrm{SiCH}_{3}\right), 0.77-0.82\left(\mathrm{~m}, 6 \mathrm{H}, 3 \times \mathrm{Sn}\left(\mathrm{CH}_{2}\right)_{2} \mathrm{CH}_{2}\right), 0.88-0.92(\mathrm{~m}, 18 \mathrm{H}$, $\left.\mathrm{SiC}\left(\mathrm{CH}_{3}\right)_{3}, 3 \times \mathrm{Sn}\left(\mathrm{CH}_{2}\right)_{3} \mathrm{CH}_{3}\right), 1.25-1.53\left(\mathrm{~m}, 16 \mathrm{H}, 3 \times \mathrm{SnCH}_{2} \mathrm{CH}_{2}, 3 \times \mathrm{SnCH}_{2}, \mathrm{H}-1, \mathrm{H}-5\right.$, two of cyclopentyl $\left.\mathrm{CH}_{2}\right), 1.60-1.64\left(\mathrm{~m}, 1 \mathrm{H}\right.$, one of cyclopentyl $\left.\mathrm{CH}_{2}\right), 1.96-2.05(\mathrm{~m}, 1 \mathrm{H}$, one of cyclopentyl $\left.\mathrm{CH}_{2}\right), 4.22$ - $4.24(\mathrm{~m}, 1 \mathrm{H}, \mathrm{H}-2) ;{ }^{13} \mathrm{C} \mathrm{NMR}\left(\mathrm{CDCl}_{3}, 100 \mathrm{MHz}\right)-4.5\left(\mathrm{SiCH}_{3}\right), 2.1(\mathrm{C}-6)$, 
$8.7\left(3 \times \mathrm{SnCH}_{2}, J_{\mathrm{Sn}-\mathrm{C}}=161\right), 13.7\left(3 \times \mathrm{Sn}\left(\mathrm{CH}_{2}\right)_{3} \mathrm{CH}_{3}\right), 18.5\left(\mathrm{SiC}\left(\mathrm{CH}_{3}\right)_{3}\right), 21.4,26.1\left(\mathrm{SiC}\left(\mathrm{CH}_{3}\right)_{3}\right)$,

$27.3\left(3 \times \mathrm{Sn}\left(\mathrm{CH}_{2}\right)_{2} \mathrm{CH}_{2}\right), 28.9,29.0,29.1\left(3 \times \mathrm{SnCH}_{2} \mathrm{CH}_{2}\right), 31.3,76.1(\mathrm{C}-2) ;{ }^{119} \mathrm{Sn} \mathrm{NMR}(93 \mathrm{MHz})$ -7.9; MS (EI+) m/z: 445.3 (62\%), 389.2 (26\%), 253.0 (8\%), 209.0 (17\%), 195.0 (29\%), 117.0 (23\%), $166.2(15 \%), 137.0$ (9\%), 121.0 (8\%); HRMS $m / z: \mathrm{M}^{+}$found 502.2647, $\mathrm{C}_{24} \mathrm{H}_{50} \mathrm{OSiSn}$ requires 502.2638 .

\section{[2-(tert-Butyldimethylsilanyloxy)bicyclo[3.1.0]hex-6-yl]phenyl methanone 24a}

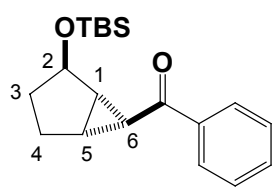

tert-Butyldimethyl-\{6-(tributylstannyl)bicyclo[3.1.0]hex-2-yloxy\}silane 23 (501 $\mathrm{mg}, 1.00 \mathrm{mmol}$ ) was reacted with a suspension of $N, N$-dimethylbenzamide (298 $\mathrm{mg}, 2.00 \mathrm{mmol}, 2.0$ equiv.) in THF (2 mL) as described in the typical procedure. Purification of the crude product by column chromatography (5\% $\mathrm{Et}_{2} \mathrm{O}$ in petrol) gave a mixture of cyclopropane $24 \mathbf{a}$ and a side-product (derived from the reaction of excess $n$-BuLi with the electrophile). Subsequent vacuum Kugelrohr distillation $\left(70{ }^{\circ} \mathrm{C}\right.$ and 0.07 mbar) gave pure cyclopropane $24 \mathrm{a}(273 \mathrm{mg}, 0.86 \mathrm{mmol}, 86 \%)$ as a white solid, $R_{\mathrm{f}}=0.3\left(5 \% \mathrm{Et}_{2} \mathrm{O}\right.$ in petrol); $\mathrm{mp}=57-58{ }^{\circ} \mathrm{C}$; IR (film) $2930 \mathrm{~s}, 2857 \mathrm{~s}(\mathrm{C}-\mathrm{H}), 1668 \mathrm{~s}$ $(\mathrm{C}=\mathrm{O}), 1599 \mathrm{~m}, 1582 \mathrm{~m}, 1449 \mathrm{~s}, 1401 \mathrm{~s}, 1360 \mathrm{~s}, 1267 \mathrm{~s}, 1220 \mathrm{~s}, 1168 \mathrm{~s}, 1097 \mathrm{~s}, 1034 \mathrm{~s} ;{ }^{1} \mathrm{H}$ NMR $(400$ MHz) 0.09 (s, 3H, $\left.\mathrm{SiCH}_{3}\right), 0.10\left(\mathrm{~s}, 3 \mathrm{H}, \mathrm{SiCH}_{3}\right), 0.91\left(\mathrm{~s}, 9 \mathrm{H}, \mathrm{SiC}\left(\mathrm{CH}_{3}\right)_{3}\right), 1.43-1.52$ (m, 1H, H-3), $1.64\left(\mathrm{dd}, 1 \mathrm{H}, \mathrm{H}-3^{\prime}, J=8.4,14.4\right), 1.83(\mathrm{dd}, 1 \mathrm{H}, \mathrm{H}-4, J=8.4,12.8), 2.11-2.24$ (m, 3H, H-4', H-5, H-6), 2.29 (t, 1H, H-6, $J=2.8), 4.37(\mathrm{~d}, 1 \mathrm{H}, \mathrm{H}-2, J=5.2), 7.47$ (t, 2H, $2 \times$ Ar-H, $J=8.0), 7.56(\mathrm{t}$, $1 \mathrm{H}, \operatorname{Ar}-\mathrm{H}, J=6.8), 7.94(\mathrm{~d}, 2 \mathrm{H}, 2 \times \mathrm{Ar}-\mathrm{H}, J=8.4) ;{ }^{13} \mathrm{C} \mathrm{NMR}(100 \mathrm{MHz})-4.7\left(\mathrm{SiCH}_{3}\right),-4.6$ $\left(\mathrm{SiCH}_{3}\right), 18.2\left(\mathrm{SiC}\left(\mathrm{CH}_{3}\right)_{3}\right), 25.5(\mathrm{C}-4), 25.9\left(\mathrm{SiC}\left(\mathrm{CH}_{3}\right)_{3}\right), 26.7(\mathrm{C}-6), 31.8(\mathrm{C}-3), 32.2(\mathrm{C}-5), 38.1$ (C-1), 74.3 (C-2), 127.9 (Ar-C), 128.4 (Ar-C), 133.0 (Ar-C), 138.0 (Ar-C), 198.8 (C=O); MS (CI+) $m / z: 334.2\left(\mathrm{MNH}_{4}{ }^{+}, 4 \%\right), 317.2\left(\mathrm{MH}^{+}, 100 \%\right), 259.1(23 \%), 185.1(20 \%), 131.1(17 \%)$; HRMS $m / z$ : $\mathrm{MNH}_{4}{ }^{+}$found 334.2203, $\mathrm{C}_{19} \mathrm{H}_{32} \mathrm{NO}_{2} \mathrm{Si}$ requires 334.2202; Elemental analysis: found $\mathrm{C}, 72.08 ; \mathrm{H}$, 8.93; $\mathrm{C}_{19} \mathrm{H}_{28} \mathrm{O}_{2} \mathrm{Si}$ requires $\mathrm{C}, 72.10 ; \mathrm{H}, 8.92$. 


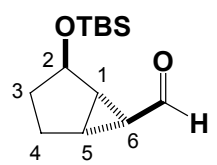

tert-Butyldimethyl-\{6-(tributylstannyl)bicyclo[3.1.0]hex-2-yloxy\}silane 23 (5012 $\mathrm{mg}, 1.00 \mathrm{mmol})$ was reacted with $N, N$-dimethylformamide $(0.15 \mathrm{~mL}, 2.00 \mathrm{mmol}, 2.0$ equiv. $)$ as described in the typical procedire. Purification of the crude product by column chromatography $\left(10 \% \mathrm{Et}_{2} \mathrm{O}\right.$ in petrol) gave cyclopropane $24 \mathbf{b}(137 \mathrm{mg}, 0.57 \mathrm{mmol}, 57 \%)$ as a colorless oil, $R_{\mathrm{f}}=0.3$ $\left(10 \% \mathrm{Et}_{2} \mathrm{O}\right.$ in petrol); IR (film) 2952s $(\mathrm{C}-\mathrm{H}), 1684 \mathrm{~s}(\mathrm{C}=\mathrm{O}), 1463 \mathrm{~s}, 1361 \mathrm{~m}, 1317 \mathrm{~m}, 1257 \mathrm{~s}, 1217 \mathrm{~m}$, 1169m, 1007s; ${ }^{1} \mathrm{H}$ NMR (400 MHz) 0.07 (s, 3H, $\mathrm{SiCH}_{3}$ ), 0.09 (s, 3H, $\mathrm{SiCH}_{3}$ ), 0.90 (s, 9H, $\left.\mathrm{SiC}\left(\mathrm{CH}_{3}\right)_{3}\right), 1.28$ - $1.37(\mathrm{~m}, 1 \mathrm{H}, \mathrm{H}-3), 1.48$ - $1.50(\mathrm{~m}, 1 \mathrm{H}, \mathrm{H}-6), 1.56\left(\mathrm{dd}, 1 \mathrm{H}, \mathrm{H}-3^{\prime}, J=8.8,14.8\right)$, $1.78(\mathrm{dd}, 1 \mathrm{H}, \mathrm{H}-4, J=8.4,12.4), 2.03(\mathrm{dd}, 1 \mathrm{H}, \mathrm{H}-1, J=3.6,5.6), 2.08-2.16\left(\mathrm{~m}, 2 \mathrm{H}, \mathrm{H}-4^{\prime}, \mathrm{H}-5\right)$, $4.32(\mathrm{~d}, 1 \mathrm{H}, \mathrm{H}-2, J=4.8), 9.04(\mathrm{~d}, 1 \mathrm{H}, \mathrm{CHO}, J=5.6) ;{ }^{13} \mathrm{C} \mathrm{NMR}(100 \mathrm{MHz})-4.7\left(\mathrm{SiCH}_{3}\right),-4.7$ $\left(\mathrm{SiCH}_{3}\right), 18.2\left(\mathrm{SiC}\left(\mathrm{CH}_{3}\right)_{3}\right), 24.9(\mathrm{C}-4), 25.8\left(\mathrm{SiC}\left(\mathrm{CH}_{3}\right)_{3}\right), 27.6(\mathrm{C}-5), 31.3(\mathrm{C}-3), 32.1(\mathrm{C}-6), 34.1$ (C-1), 74.0 (C-2), $200.0(\mathrm{C}=\mathrm{O})$; MS (CI+) m/z: $258.2\left(\mathrm{MNH}_{4}{ }^{+}, 26 \%\right), 241.2\left(\mathrm{MH}^{+}, 100 \%\right), 200.1$ (11\%), 183.1 (15\%), 126.1 (5\%), 109.1 (15\%); HRMS m/z: $\mathrm{MNH}_{4}{ }^{+}$found $258.1877, \mathrm{C}_{13} \mathrm{H}_{28} \mathrm{NO}_{2} \mathrm{Si}$ requires 258.1889 .

\section{1-[2-(tert-Butyldimethylsilanyloxy)bicyclo[3.1.0]hex-6-yl]propan-1-one 24c}

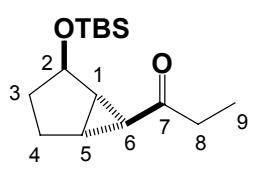

tert-Butyldimethyl-\{6-(tributylstannyl)bicyclo[3.1.0]hex-2-yloxy\}silane 23 (501 $\mathrm{mg}, 1.00 \mathrm{mmol})$ was reacted with $N, N$-dimethylpropioamide $(0.22 \mathrm{~mL}, 2.00 \mathrm{mmol}, 2.0$ equiv. $)$ as described in the typical procedure. Purification of the crude product by column chromatography $\left(10 \% \mathrm{Et}_{2} \mathrm{O}\right.$ in petrol) gave a mixture of cyclopropane $\mathbf{2 4 c}$ and a side-product (derived from the reaction of excess $n$-BuLi with the electrophile). Subsequent vacuum Kugelrohr distillation $\left(60{ }^{\circ} \mathrm{C}\right.$ and $10 \mathrm{mbar})$ gave pure cyclopropane $24 \mathrm{c}(161 \mathrm{mg}, 0.62 \mathrm{mmol}, 62 \%)$ as a colorless oil, $R_{\mathrm{f}}=0.3$ $\left(10 \% \mathrm{Et}_{2} \mathrm{O}\right.$ in petrol); IR (film) 2932s, 2858s (C-H), 1702s $(\mathrm{C}=\mathrm{O}), 1462 \mathrm{~m}, 1406 \mathrm{~m}, 1360 \mathrm{~m}, 1256 \mathrm{~s}$, $1171 \mathrm{~s}, 1124 \mathrm{~s}, 1097 \mathrm{~m}, 1034 \mathrm{~s} ;{ }^{1} \mathrm{H}$ NMR (400 MHz) 0.06 (s, 3H, $\left.\mathrm{SiCH}_{3}\right), 0.07$ (s, 3H, $\left.\mathrm{SiCH}_{3}\right), 0.88$ (s, 9H, $\left.\mathrm{SiC}\left(\mathrm{CH}_{3}\right)_{3}\right), 1.05$ (t, 3H, $\left.3 \times \mathrm{H}-9, J=7.2\right), 1.27$ - $1.37(\mathrm{~m}, 1 \mathrm{H}, \mathrm{H}-3), 1.51$ - 1.56 (m, 2H, H-3', H-6), $1.71(\mathrm{dd}, 1 \mathrm{H}, \mathrm{H}-4, J=8.0,12.8), 1.92-2.10\left(\mathrm{~m}, 3 \mathrm{H}, \mathrm{H}-1, \mathrm{H}-4{ }^{\prime}, \mathrm{H}-5\right), 2.50(\mathrm{q}, 2 \mathrm{H}, 2 \times \mathrm{H}-8, J$ 
= 7.2), $4.27(\mathrm{~d}, 1 \mathrm{H}, \mathrm{H}-2, J=4.4) ;{ }^{13} \mathrm{C} \mathrm{NMR}(100 \mathrm{MHz})-4.7\left(\mathrm{SiCH}_{3}\right),-4.6\left(\mathrm{SiCH}_{3}\right), 7.9(\mathrm{C}-9), 18.2$ $\left(\mathrm{SiC}\left(\mathrm{CH}_{3}\right)_{3}\right), 25.3(\mathrm{C}-4), 25.9\left(\mathrm{SiC}\left(\mathrm{CH}_{3}\right)_{3}\right), 29.8(\mathrm{C}-5), 30.5(\mathrm{C}-6), 31.6(\mathrm{C}-3), 36.5(\mathrm{C}-8), 36.8(\mathrm{C}-$ 1), $74.3(\mathrm{C}-2), 209.5(\mathrm{C}=\mathrm{O})$; $\mathrm{MS}(\mathrm{CI}+) \mathrm{m} / z: 286.2\left(\mathrm{MNH}_{4}^{+}, 15 \%\right), 269.2\left(\mathrm{MH}^{+}, 100 \%\right), 228.1$ (24\%), 211.1 (34\%), 154.1 (29\%), 137.1 (74\%); HRMS m/z: $\mathrm{MNH}_{4}{ }^{+}$found 286.2205, $\mathrm{C}_{15} \mathrm{H}_{32} \mathrm{NO}_{2} \mathrm{Si}$ requires 286.2202 .

\section{[2-(tert-Butyldimethylsilanyloxy)bicyclo[3.1.0]hex-6-yl]phenyl methanol 24d}

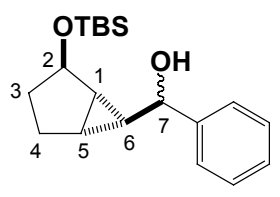

tert-Butyldimethyl-\{6-(tributylstannyl)bicyclo[3.1.0]hex-2-yloxy\}silane 23 (501 $\mathrm{mg}, 1.00 \mathrm{mmol})$ was reacted with benzamide $(0.20 \mathrm{~mL}, 2.00 \mathrm{mmol}, 2.0$ equiv. $)$ as described in the typical procedure. Purification of the crude product by column chromatography $\left(20 \% \mathrm{Et}_{2} \mathrm{O}\right.$ in petrol) gave two diastereoisomers, both contaminated with the side-product (derived from the reaction of excess $n$-BuLi with the electrophile). Subsequent vacuum Kugelrohr distillation $\left(95{ }^{\circ} \mathrm{C}\right.$ and $0.07 \mathrm{mbar})$ gave pure diastereomer A (108 $\mathrm{mg}, 0.34 \mathrm{mmol}, 34 \%)$ as a pale yellow oil, $R_{\mathrm{f}}=0.2$ $\left(20 \% \mathrm{Et}_{2} \mathrm{O}\right.$ in petrol), and diastereomer B $(80 \mathrm{mg}, 0.25 \mathrm{mmol}, 25 \%)$ as a pale yellow oil, $R_{\mathrm{f}}=0.1$ $\left(20 \% \mathrm{Et}_{2} \mathrm{O}\right.$ in petrol).

\section{Characterisation data for diastereomer A:}

IR (film) 3384bs (O-H), 3030w (Ar), 2928s, 2856s (C-H), 1494w, 1462s, 1361s, 1255s, 1169s, 1071s; ${ }^{1} \mathrm{H}$ NMR (400 MHz) 0.09 (s, 3H, $\mathrm{SiCH}_{3}$ ), 0.11 (s, 3H, $\mathrm{SiCH}_{3}$ ), $0.84-0.88$ (m, 1H, H-6), 0.92 (s, 9H, SiC(CH3) $\left.)_{3}\right), 1.27$ - 1.34 (m, 1H, H-3), 1.48 - 1.64 (m, 4H, H-1, H-3', H-4, H-5), 1.92 (d, 1H, $\mathrm{OH}, J=3.2), 1.94-2.00\left(\mathrm{~m}, 1 \mathrm{H}, \mathrm{H}-4^{\prime}\right), 4.05(\mathrm{dd}, 1 \mathrm{H}, \mathrm{H}-7, J=2.8,8.4), 4.32(\mathrm{~d}, 1 \mathrm{H}, \mathrm{H}-2, J=4.4)$, 7.29 - $7.41(\mathrm{~m}, 5 \mathrm{H}, 5 \times \mathrm{Ar}-\mathrm{H}) ;{ }^{13} \mathrm{C} \mathrm{NMR}(100 \mathrm{MHz})-4.5\left(\mathrm{SiCH}_{3}\right), 18.2\left(\mathrm{SiC}\left(\mathrm{CH}_{3}\right)_{3}\right), 22.7(\mathrm{C}-5)$, 24.7 (C-4), $26.0\left(\mathrm{SiC}\left(\mathrm{CH}_{3}\right)_{3}\right), 27.9$ (C-6), $31.2(\mathrm{C}-1), 32.2$ (C-3), 74.5 (C-2), 76.8 (C-7), 125.8 (ArC), 127.5 (Ar-C), 128.4 (Ar-C), 143.8 (Ar-C); MS (CI+) m/z: $336.3\left(\mathrm{MNH}_{4}^{+}, 1 \%\right), 319.2\left(\mathrm{MH}^{+}\right.$, 2\%), 301.2 (16\%), 260.2 (4\%), 243.1 (8\%), 204.1 (5\%), $187.1(40 \%), 169.1(100 \%), 143.1(6 \%)$, 115.1 (3\%); HRMS $m / z$ : $\mathrm{MNH}_{4}{ }^{+}$found 336.2355, $\mathrm{C}_{19} \mathrm{H}_{34} \mathrm{NO}_{2} \mathrm{Si}$ requires 336.2359. 
Characterisation data for diastereomer B:

IR (film) 3357bs (O-H), 3030w (Ar), 2929s (C-H), 1361s, 1255s, 1169s, 1039s; ${ }^{1} \mathrm{H}$ NMR (400 MHz) 0.05 (s, 3H, $\left.\mathrm{SiCH}_{3}\right), 0.06\left(\mathrm{~s}, 3 \mathrm{H}, \mathrm{SiCH}_{3}\right), 0.83$ - $0.86(\mathrm{~m}, 1 \mathrm{H}, \mathrm{H}-6), 0.89\left(\mathrm{~s}, 9 \mathrm{H}, \mathrm{SiC}\left(\mathrm{CH}_{3}\right)_{3}\right)$, 1.28 - 1.34 (m, 1H, H-3), 1.37 (dd, 1H, H-1, $J=3.2,6.0), 1.51$ (dd, 1H, H-3', $J=8.4,14.4), 1.64$ 1.68 (m, 1H, H-5), 1.74 (dd, 1H, H-4, $J=8.8,13.2), 2.00(\mathrm{~d}, 1 \mathrm{H}, \mathrm{OH}, J=2.8), 1.98-2.04(\mathrm{~m}, 1 \mathrm{H}$, $\left.\mathrm{H}^{-} 4^{\prime}\right), 4.08(\mathrm{dd}, 1 \mathrm{H}, \mathrm{H}-7, J=2.4,8.4), 4.17(\mathrm{~d}, 1 \mathrm{H}, \mathrm{H}-2, J=4.8), 7.28-7.42(\mathrm{~m}, 5 \mathrm{H}, 5 \times \mathrm{Ar}-\mathrm{H}) ;{ }^{13} \mathrm{C}$ NMR (100 MHz) -4.6 ( $\left.\mathrm{SiCH}_{3}\right), 18.2\left(\mathrm{SiC}\left(\mathrm{CH}_{3}\right)_{3}\right), 23.5(\mathrm{C}-5), 25.1(\mathrm{C}-4), 25.9\left(\mathrm{SiC}\left(\mathrm{CH}_{3}\right)_{3}\right), 27.9(\mathrm{C}-$ 6), 30.4 (C-1), 32.3 (C-3), 74.4 (C-2), 76.5 (C-7), 125.8 (Ar-C), 127.5 (Ar-C), 128.3 (Ar-C), 143.9 (Ar-C); MS (CI+) m/z: $336.2\left(\mathrm{MNH}_{4}^{+}, 1 \%\right), 319.2\left(\mathrm{MH}^{+}, 5 \%\right), 301.2(100 \%), 261.1(11 \%), 243.1$ (9\%), 204.1 (9\%), 187.1 (75\%), 169.1 (81\%), 143.1 (9\%); HRMS $m / z: \mathrm{MNH}_{4}{ }^{+}$found 336.2355, $\mathrm{C}_{19} \mathrm{H}_{34} \mathrm{NO}_{2} \mathrm{Si}$ requires 336.2359 .

\section{1-[2-(tert-Butyldimethylsilanyloxy)bicyclo[3.1.0]hex-6-yl]cyclohexanol 24e}

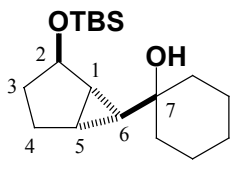

tert-Butyldimethyl-\{6-(tributylstannyl)bicyclo[3.1.0]hex-2-yloxy\} silane 23 (501 mg, $1.00 \mathrm{mmol})$ was reacted with cyclohexanone $(0.21 \mathrm{~mL}, 2.00 \mathrm{mmol}, 2.0$ equiv. $)$ as described in the typical procedure. Purification of the crude product by column chromatography $\left(20 \% \mathrm{Et}_{2} \mathrm{O}\right.$ in petrol) gave a mixture of cyclopropane 24e and a side-product (derived from the reaction of excess $n$-BuLi with the electrophile). Subsequent vacuum Kugelrohr distillation $\left(65{ }^{\circ} \mathrm{C}\right.$ and 0.07 mbar) gave pure cyclopropane $24 \mathrm{e}(186 \mathrm{mg}, 0.60 \mathrm{mmol}, 60 \%)$ as a colorless oil; $R_{\mathrm{f}}=0.2\left(20 \% \mathrm{Et}_{2} \mathrm{O}\right.$ in petrol); IR (film) 3423bs (O-H), 2934s, 2858s (C-H), 1462m, 1360m, 1312w, 1254s, 1178s, 1079s, 1041s, 1004s; ${ }^{1} \mathrm{H}$ NMR (400 MHz) 0.07 (s, 3H, $\mathrm{SiCH}_{3}$ ), 0.08 (s, 3H, SiCH $), 0.48$ (t, 1H, H-6, J= 4.0), $0.90\left(\mathrm{~s}, 9 \mathrm{H}, \mathrm{SiC}\left(\mathrm{CH}_{3}\right)_{3}\right), 0.94(\mathrm{~s}, 1 \mathrm{H}, \mathrm{OH}), 1.18$ - $1.62(\mathrm{~m}, 15 \mathrm{H}), 1.95$ - $2.02(\mathrm{~m}, 1 \mathrm{H}), 4.22(\mathrm{~d}$, $1 \mathrm{H}, \mathrm{H}-2, J=4.8) ;{ }^{13} \mathrm{C}$ NMR $(100 \mathrm{MHz})-4.5\left(\mathrm{SiCH}_{3}\right), 18.3\left(\mathrm{SiC}\left(\mathrm{CH}_{3}\right)_{3}\right), 20.0(\mathrm{C}-5), 21.9\left(\mathrm{CH}_{2}\right)$, $25.3\left(\mathrm{CH}_{2}\right), 25.8\left(\mathrm{CH}_{2}\right), 26.0\left(\mathrm{SiC}\left(\mathrm{CH}_{3}\right)_{3}\right), 27.8(\mathrm{C}-1), 30.7(\mathrm{C}-6), 32.6\left(\mathrm{CH}_{2}\right), 37.2\left(\mathrm{CH}_{2}\right), 37.3$ $\left(\mathrm{CH}_{2}\right), 69.7(\mathrm{C}-7), 75.3(\mathrm{C}-2)$; $\mathrm{MS}(\mathrm{CI}+) \mathrm{m} / z: 293.2\left(\left[\mathrm{M}-\mathrm{H}_{2} \mathrm{O}\right] \mathrm{H}^{+}, 3 \%\right), 235.2(3 \%), 161.1(100 \%)$, $134.1(11 \%)$; HRMS $m / z$ : $\left[\mathrm{M}-\mathrm{H}_{2} \mathrm{O}\right] \mathrm{H}^{+}$found 293.2295, $\mathrm{C}_{19} \mathrm{H}_{33} \mathrm{OSi}$ requires 293.2301. 


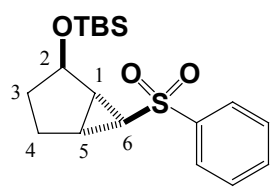

tert-Butyldimethyl-\{6-(tributylstannyl)bicyclo[3.1.0]hex-2-yloxy\} silane 23 (501 $\mathrm{mg}, 1.00 \mathrm{mmol})$ was reacted with benzenesulfonyl floride $(0.24 \mathrm{~mL}, 2.00 \mathrm{mmol}, 2.0$ equiv. $)$ as described in the typical procedure. Purification of the crude product by column chromatography $\left(20 \% \mathrm{Et}_{2} \mathrm{O}\right.$ in petrol) gave pure cyclopropane $\mathbf{2 4 f}(176 \mathrm{mg}, 0.50 \mathrm{mmol}, 50 \%)$ as a white solid, $R_{\mathrm{f}}=$ $0.2\left(20 \% \mathrm{Et}_{2} \mathrm{O}\right.$ in petrol); $\mathrm{mp}=72-73{ }^{\circ} \mathrm{C}$; IR (KBr) 3063w (Ar), 2954s, 2895s, 2857s (C-H), $1472 \mathrm{~m}, 1447 \mathrm{~m}, 1360 \mathrm{~m}, 1308 \mathrm{~s}, 1256 \mathrm{~m}, 1169 \mathrm{~m}, 1149 \mathrm{~s}, 1087 \mathrm{~s}, 1031 \mathrm{~s} ;{ }^{1} \mathrm{H}$ NMR (400 MHz) 0.04 (s, $\left.3 \mathrm{H}, \mathrm{SiCH}_{3}\right), 0.06\left(\mathrm{~s}, 3 \mathrm{H}, \mathrm{SiCH}_{3}\right), 0.87\left(\mathrm{~s}, 9 \mathrm{H}, \mathrm{SiC}\left(\mathrm{CH}_{3}\right)_{3}\right), 1.15-1.24(\mathrm{~m}, 1 \mathrm{H}, \mathrm{H}-3), 1.53$ (dd, 1H, H$\left.3^{\prime}, J=8.4,14.4\right), 1.69(\mathrm{dd}, 1 \mathrm{H}, \mathrm{H}-4, J=7.6,13.2), 2.00(\mathrm{t}, 1 \mathrm{H}, \mathrm{H}-6, J=3.6), 2.03-2.12(\mathrm{~m}, 1 \mathrm{H}, \mathrm{H}-$ $\left.4^{\prime}\right), 2.21(\mathrm{dd}, 1 \mathrm{H}, \mathrm{H}-1, J=3.2,7.2), 2.29-2.32(\mathrm{~m}, 1 \mathrm{H}, \mathrm{H}-5), 4.25(\mathrm{~d}, 1 \mathrm{H}, \mathrm{H}-2, J=4.8), 7.55$ (t, 2H, $2 \times m \mathrm{Ar}-\mathrm{H}, J=7.2), 7.64(\mathrm{t}, 1 \mathrm{H}, p \mathrm{Ar}-\mathrm{H}, J=7.2), 7.88(\mathrm{~d}, 2 \mathrm{H}, 2 \times o \mathrm{Ar}-\mathrm{H}, J=6.8) ;{ }^{1} \mathrm{H}$ NMR $(100$

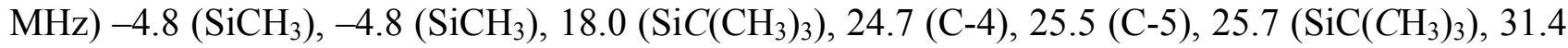
(C-3), 32.0 (C-1), 40.0 (C-6), 73.6 (C-2), 127.3 (Ar-C), 129.2 (Ar-C), 133.3 (Ar-C), 140.9 (Ar-C); Elemental analysis: found $\mathrm{C}, 61.48 ; \mathrm{H}, 8.05 ; \mathrm{C}_{18} \mathrm{H}_{28} \mathrm{O}_{3} \mathrm{SSi}$ requires $\mathrm{C}, 61.32 ; \mathrm{H}, 8.00$.

\section{2-(tert-Butyldimethylsilanyloxy)bicyclo[3.1.0]hexan-6-ol $24 \mathrm{~g}$}

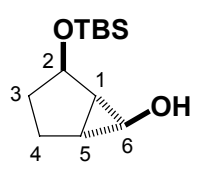

$n$-BuLi (1.6 M in hexane, $0.6 \mathrm{~mL}, 1.0 \mathrm{mmol}, 1.1$ equiv.) was added to a stirred solution of tert-butyldimethyl-\{6-(tributylstannyl)bicyclo[3.1.0]hex-2-yloxy\}silane 23 (450 mg, $0.90 \mathrm{mmol})$ in THF $(5 \mathrm{~mL})$ at $0{ }^{\circ} \mathrm{C}$. After $1 \mathrm{~h}$, the mixture was cooled to $-78{ }^{\circ} \mathrm{C}$, treated with a suspension of $\mathrm{MoOPH}^{[14]}$ (586 mg, $1.35 \mathrm{mmol}, 1.5$ equiv.) in THF $(1.5 \mathrm{~mL})$ and then stirred at the same temperature for a further $3 \mathrm{~h}$. The resulting mixture was warmed to $\mathrm{rt}$, dry-loaded onto a small amount of silica and purified by column chromatography $\left(40 \% \mathrm{Et}_{2} \mathrm{O}\right.$ in petrol) to give cyclopropylalcohol $\mathbf{2 4 g}(125 \mathrm{mg}, 0.55 \mathrm{mmol}, 61 \%)$ as a colorless oil, $R_{\mathrm{f}}=0.3\left(40 \% \mathrm{Et}_{2} \mathrm{O}\right.$ in petrol); IR (film) 3319bs (O-H), 2930s, 2858s (C-H), 1463s, 1362s, 1319m, 1256s, 1168s, 1133s, 1076s, 1034s, 1012s; ${ }^{1} \mathrm{H}$ NMR (400 MHz) 0.07 (s, 3H, $\left.\mathrm{SiCH}_{3}\right), 0.08$ (s, 3H, $\left.\mathrm{SiCH}_{3}\right), 0.89$ (s, 9H, 
$\left.\mathrm{SiC}\left(\mathrm{CH}_{3}\right)_{3}\right), 1.00-1.09(\mathrm{~m}, 1 \mathrm{H}, \mathrm{H}-3), 1.43-1.50\left(\mathrm{~m}, 2 \mathrm{H}, \mathrm{H}-1, \mathrm{H}-3{ }^{\prime}\right), 1.57$ (t, 1H, H-5, $\left.J=6.4\right)$, $1.68(\mathrm{dd}, 1 \mathrm{H}, \mathrm{H}-4, J=8.4,12.4), 1.89-1.98$ (m, 2H, H-4', OH), 3.02 (s, 1H, H-6), 4.25 (d, 1H, H-

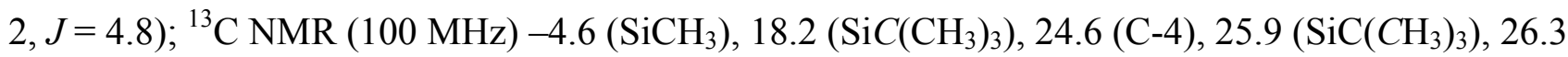
(C-5), 33.4 (C-3), 34.3 (C-1), 53.4 (C-6), 73.8 (C-2); MS (CI+) m/z: $229.2\left(\mathrm{MH}^{+}, 100 \%\right), 171.1$ (34\%); HRMS $m / z: \mathrm{MH}^{+}$found 229.1616, $\mathrm{C}_{12} \mathrm{H}_{25} \mathrm{O}_{2} \mathrm{Si}$ requires 229.1624.

\section{tert-Butyldimethyl-(5-tributylstannylbicyclo[3.1.0]hex-2-yloxy)silane 25}

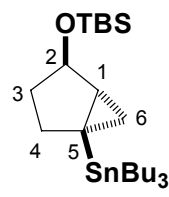

tert-Butyldimethylsilyl chloride (226 mg, $1.50 \mathrm{mmol}, 1.5$ equiv.) was added to a stirred solution of 5-tributylstannylbicyclo[3.1.0]hexan-2-ol 5f (387 mg, $1.00 \mathrm{mmol})$ and imidazole (136 $\mathrm{mg}, 2.00 \mathrm{mmol}, 2.0$ equiv.) in $\mathrm{CH}_{2} \mathrm{Cl}_{2}(10 \mathrm{~mL})$ at $\mathrm{rt}$, and then stirred for $4 \mathrm{~h}$. The reaction mixture was diluted with $\mathrm{CH}_{2} \mathrm{Cl}_{2}(100 \mathrm{~mL})$ then washed with sat. aq. $\mathrm{NaHCO}_{3}(20 \mathrm{~mL})$. The layers were separated and the aqueous layer extracted with $\mathrm{CH}_{2} \mathrm{Cl}_{2}(20 \mathrm{~mL})$. The combined organic layers were dried $\left(\mathrm{MgSO}_{4}\right)$, filtered and concentrated to give the crude product. Purification by column chromatography (3\% $\mathrm{Et}_{2} \mathrm{O}$ in petrol) gave protected alcohol 25 (456 $\left.\mathrm{mg}, 0.91 \mathrm{mmol}, 91 \%\right)$ as a colorless oil; $R_{\mathrm{f}}=0.6\left(10 \% \mathrm{Et}_{2} \mathrm{O}\right.$ in petrol); IR (film) $2957 \mathrm{~s}, 2927 \mathrm{~s}, 2856 \mathrm{~s}(\mathrm{C}-\mathrm{H}), 1463 \mathrm{~m}, 1362 \mathrm{~m}$, 1254s, 1089s, 1070s, 1038s; ${ }^{1} \mathrm{H}$ NMR (500 MHz) 0.06 (s, 3H, $\left.\mathrm{SiCH}_{3}\right), 0.07$ (s, 3H, $\left.\mathrm{SiCH}_{3}\right), 0.10$ (t, $1 \mathrm{H}, \mathrm{H}-6, J=4.0), 0.26\left(\mathrm{dd}, 1 \mathrm{H}, \mathrm{H}-6^{\prime}, J=5.5,8.0\right), 0.82\left(\mathrm{t}, 6 \mathrm{H}, 3 \times \mathrm{Sn}\left(\mathrm{CH}_{2}\right)_{2} \mathrm{CH}_{2}, J=8.0\right), 0.89-$ $0.92\left(\mathrm{~m}, 18 \mathrm{H}, 3 \times \mathrm{Sn}\left(\mathrm{CH}_{2}\right)_{3} \mathrm{CH}_{3}, \mathrm{SiC}\left(\mathrm{CH}_{3}\right)_{3}\right), 1.24-1.56\left(\mathrm{~m}, 15 \mathrm{H}, 3 \times \mathrm{SnCH}_{2}, 3 \times \mathrm{SnCH}_{2} \mathrm{CH}_{2}, \mathrm{H}-1\right.$, $2 \times \mathrm{H}-3), 1.68(\mathrm{dd}, 1 \mathrm{H}, \mathrm{H}-4, J=7.5,11.5), 1.87-1.93\left(\mathrm{~m}, 1 \mathrm{H}, \mathrm{H}-4^{\prime}\right), 4.33(\mathrm{~d}, 1 \mathrm{H}, \mathrm{H}-2, J=4.5) ;{ }^{13} \mathrm{C}$ NMR $(125 \mathrm{MHz})-4.6\left(\mathrm{SiCH}_{3}\right), 8.4\left(3 \times \mathrm{SnCH}_{2}, J_{\mathrm{Sn}-\mathrm{C}}=164\right), 10.4(\mathrm{C}-6), 12.6(\mathrm{C}-5), 13.7(3 \times$ $\left.\mathrm{Sn}\left(\mathrm{CH}_{2}\right)_{3} \mathrm{CH}_{3}\right), 18.1\left(\mathrm{SiC}\left(\mathrm{CH}_{3}\right)_{3}\right), 25.9\left(\mathrm{SiC}\left(\mathrm{CH}_{3}\right), 27.5\left(3 \times \mathrm{Sn}\left(\mathrm{CH}_{2}\right)_{2} \mathrm{CH}_{2}\right), 29.0(\mathrm{C}-1), 29.1(3 \times\right.$ $\left.\mathrm{SnCH}_{2} \mathrm{CH}_{2}\right), 30.7$ (C-3), 30.8 (C-4), $75.2(\mathrm{C}-2) ;{ }^{119} \mathrm{Sn}$ NMR (125 MHz) -9.8; MS (CI-, $\left.{ }^{120} \mathrm{Sn}\right) \mathrm{m} / z$ : $501.3\left([\mathrm{M}-\mathrm{H}]^{-}, 56 \%\right), 467.1(9 \%), 419.3(28 \%), 387.3(85 \%), 361.1(56 \%), 313.1(100 \%), 291.2$ (29\%), $269.1(29 \%), 250.1(9 \%), 222.1(24 \%)$; HRMS $m / z:\left([M-H]^{-}\right)$found 497.2574, $\mathrm{C}_{24} \mathrm{H}_{49} \mathrm{O}^{116} \mathrm{Sn}$ requires 497.2576 . 


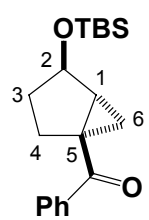

tert-Butyldimethyl-(6-tributylstannylbicyclo[3.1.0]hex-2-yloxy)silane 25 (501 mg, 1.00 mmol) was reacted with a suspension of $N, N$-dimethylbenzamide (298 $\mathrm{mg}, 2.00 \mathrm{mmol}, 2.0$ equiv.) in THF $(2 \mathrm{~mL})$ as described in the typical procedure. Purification of the crude product by column chromatography $\left(10 \% \mathrm{Et}_{2} \mathrm{O}\right.$ in petrol) gave a mixture of cyclopropane $\mathbf{2 6}$ and a side-product (derived from the reaction of excess $n$-BuLi with the electrophile). Subsequent vacuum Kugelrohr distillation $\left(70{ }^{\circ} \mathrm{C}\right.$ and $\left.0.07 \mathrm{mmbar}\right)$ gave pure cyclopropane $26(260 \mathrm{mg}, 0.82 \mathrm{mmol}, 82 \%)$ as a colorless oil, $R_{\mathrm{f}}=0.3\left(10 \% \mathrm{Et}_{2} \mathrm{O}\right.$ in petrol); IR (film) $3062 \mathrm{~m}, 2955 \mathrm{~s}(\mathrm{C}-\mathrm{H}), 1669 \mathrm{~s}(\mathrm{C}=\mathrm{O}), 1599 \mathrm{~s}$, $1580 \mathrm{~m}, 1471 \mathrm{~s}, 1448 \mathrm{~s}, 1380 \mathrm{~s}, 1342 \mathrm{~s}, 1313 \mathrm{~s}, 1295 \mathrm{~s}, 1257 \mathrm{~s}, 1199 \mathrm{~s}, 1181 \mathrm{~s} ;{ }^{1} \mathrm{H}$ NMR (400 MHz) 0.14 $\left(\mathrm{s}, 6 \mathrm{H}, 2 \times \mathrm{SiCH}_{3}\right), 0.61(\mathrm{t}, 1 \mathrm{H}, \mathrm{H}-6, J=4.8), 0.97\left(\mathrm{~s}, 9 \mathrm{H}, \mathrm{SiC}\left(\mathrm{CH}_{3}\right)_{3}\right), 1.58\left(\mathrm{dd}, 1 \mathrm{H}, \mathrm{H}-6^{\prime}, J=3.2\right.$, 5.2), $1.60-1.61(\mathrm{~m}, 1 \mathrm{H}, \mathrm{H}-3), 1.75\left(\mathrm{dd}, 1 \mathrm{H}, \mathrm{H}-3^{\prime}, J=8.4,14.0\right), 1.92$ (dd, 1H, H-4, $\left.J=8.0,12.4\right)$, $1.98(\mathrm{dd}, 1 \mathrm{H}, \mathrm{H}-1, J=4.8,8.8), 2.48-2.56\left(\mathrm{~m}, 1 \mathrm{H}, \mathrm{H}-4^{\prime}\right), 4.39(\mathrm{~d}, 1 \mathrm{H}, \mathrm{H}-2, J=4.8), 7.41(\mathrm{~m}, 2 \mathrm{H}, 2$ $\times m \mathrm{Ar}-\mathrm{H}), 7.48-7.52(\mathrm{~m}, 1 \mathrm{H}, p \mathrm{Ar}-\mathrm{H}), 8.06(\mathrm{dd}, 2 \mathrm{H}, 2 \times o \mathrm{Ar}-\mathrm{H}, J=1.2,8.4) ;{ }^{13} \mathrm{C} \mathrm{NMR}(100 \mathrm{MHz})$ -4.7 $\left(\mathrm{SiCH}_{3}\right), 13.2(\mathrm{C}-6), 18.1\left(\mathrm{SiC}\left(\mathrm{CH}_{3}\right)_{3}\right), 25.8\left(\mathrm{SiC}\left(\mathrm{CH}_{3}\right)_{3}\right), 28.9(\mathrm{C}-4), 32.8(\mathrm{C}-3), 38.0(\mathrm{C}-1)$, 38.1 (C-5), 47.7 (C-2), 128.1 (Ar-C), 128.8 (Ar-C), 131.8 (Ar-C), 137.9 (Ar-C), 203.0 (C=O); MS (CI+) $m / z: 334.2\left(\mathrm{MNH}_{4}^{+}, 6 \%\right), 317.2\left(\mathrm{MH}^{+}, 40 \%\right), 259.1(63 \%), 202.1(4 \%), 185.1(100 \%), 105.0$ (19\%); HRMS $m / z: \mathrm{MNH}_{4}{ }^{+}$found 334.2206, $\mathrm{C}_{19} \mathrm{H}_{32} \mathrm{NO}_{2}$ Si requires 334.2202.

\section{Stille cross-coupling of cyclopropylstannane 23 with 4-chloroanisole}

A mixture of 23 (1.0 mmol), 4-chloroanisole (1.2 equiv.), $\mathrm{Pd}_{2}(\mathrm{dba})_{3}(1.5 \mathrm{~mol} \%),\left[(t-\mathrm{Bu})_{3} \mathrm{PH}^{\mathrm{B}} \mathrm{BF}_{4}(6\right.$ mol\%), CsF (2.2 equiv.) and ethyl diisopropylamine (6 mol\%) in dioxane $(2 \mathrm{~mL})$ was heated in a sealed tube at $100{ }^{\circ} \mathrm{C}$ for $48 \mathrm{~h}$. Under these conditions, incomplete consumption of the starting cyclopropylstanne was observed (entry 1). The yield based on recovered starting material (brsm) improved with a reaction duration of $72 \mathrm{~h}$ (entry 2), while use of a higher catalytic loading resulted in similar yield of product, but with much less recovered starting material (entry 3). Surprisingly, no reaction was observed between $\mathbf{2 3}$ and the more reactive 4-bromobenzonitrile (entry 4). The $\mathrm{Pd} / \mathrm{P} t$ - 
$\mathrm{Bu}_{3}$ catalytic system was previously shown to be stable in a microwave assisted cross-coupling reaction between aryl halides and aryl zinc halides. ${ }^{[15]}$ Therefore we considered worthwhile to conduct the coupling reaction under microwave irradiation. Coupled adduct $\mathbf{2 7}$ was isolated in $35 \%$ yield (95\% yield brsm) after irradiation at $75 \mathrm{~W} / 110{ }^{\circ} \mathrm{C}$ for $15 \min$ (entry 5). No improvement was observed with a higher catalyst loading ( $4 \mathrm{~mol} \%)$ and a longer irradiation period (entry 6$)$. Although incomplete reaction was observed in each case, nonetheless the direct functionalization of precursor $\mathbf{2 3}$ via Stille cross-coupling was shown to be viable.

Table 2. Stille cross-coupling reactions

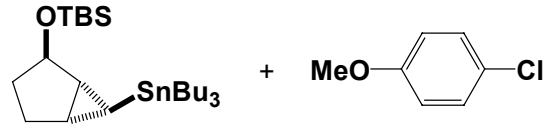

23

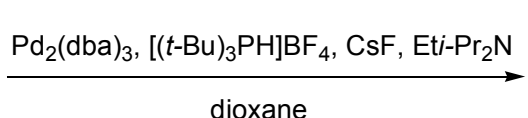

$100{ }^{\circ} \mathrm{C}$ or microwave

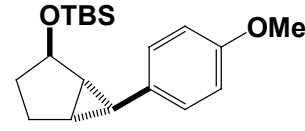

27

\begin{tabular}{ccccccc}
\hline Entry $^{a}$ & $\begin{array}{c}\mathrm{Pd}_{2}(\mathrm{dba})_{3} \\
(\mathrm{~mol} \%)\end{array}$ & $\begin{array}{c}{\left[(t-\mathrm{Bu})_{3} \mathrm{PH}\right] \mathrm{BF}_{4}} \\
(\mathrm{~mol} \%)\end{array}$ & $\begin{array}{c}\text { Microwave }^{c} \\
\text { Conditions }\end{array}$ & $\begin{array}{c}\text { Time } \\
(\mathrm{h})\end{array}$ & $\begin{array}{c}\mathbf{2 3}^{d} \\
(\%)\end{array}$ & $\begin{array}{c}\mathbf{2 7}^{d} \\
(\%)\end{array}$ \\
\hline 1 & 1.5 & 6.0 & - & 48 & 40 & 33 \\
2 & 1.5 & 6.0 & - & 72 & 44 & 48 \\
3 & 5.0 & 20.0 & - & 72 & 4 & 37 \\
$4^{b}$ & 1.5 & 6.0 & - & 72 & 100 & - \\
5 & 2.0 & 8.0 & $75 \mathrm{~W} / 110^{\circ} \mathrm{C}$ & 0.25 & 63 & 35 \\
6 & 4.0 & 16.0 & $75 \mathrm{~W} / 120{ }^{\circ} \mathrm{C}$ & 1 & 37 & 43 \\
\hline
\end{tabular}

${ }^{a}$ All reactions were performed with $23(1.0 \mathrm{mmol}), p$-4-chloroanisole (1.2 equiv.), $\mathrm{Et}^{i} \mathrm{Pr}_{2} \mathrm{~N}$ \{same as $\left[(t-\mathrm{Bu})_{3} \mathrm{PH} \mathrm{BF}{ }_{4}\right.$ \} and $\mathrm{CsF}$ (2.2 equiv.) in dioxane $(2 \mathrm{~mL})$ at $100{ }^{\circ} \mathrm{C}$. After the reaction, the mixture was dry-loaded onto a small amount of silica and purified by column chromatography $\left(2 \% \mathrm{Et}_{2} \mathrm{O}\right.$ in petrol). Unreacted 23 was separated from the chromatographed material via vacuum Kugelrohr distillation $\left(65{ }^{\circ} \mathrm{C}, 0.06\right.$ mbar) to give pure product $27 .{ }^{b}$ Reaction performed with $p$-4-bromobenzonitrile (1.2 equiv.). ${ }^{c}$ Reactor type: CEM Discover. ${ }^{d}$ Isolated yields.

tert-Butyl-[6-(4-methoxyphenyl)bicyclo[3.1.0]hex-2-yloxy]dimethylsilane 27<smiles>COc1ccc(C2CC3C(OC)CCC23)cc1</smiles>

Procedure for Entry 2, Table 2: A suspension of tert-butyldimethyl-\{6(tributylstannyl)bicyclo[3.1.0]hex-2-yloxy\}silane 23 (501 mg, $1.00 \mathrm{mmol}), \mathrm{Pd}_{2}(\mathrm{dba})_{3}(1.5 \mathrm{~mol} \%$ ), $\left[(t-\mathrm{Bu})_{3} \mathrm{PH}\right] \mathrm{BF}_{4}(6 \mathrm{~mol} \%), \mathrm{CsF}(334 \mathrm{mg}, 2.2$ equiv. $)$ and $i-\mathrm{Pr}_{2} \mathrm{EtN}(6 \mathrm{~mol} \%)$ in dioxane $(2 \mathrm{~mL})$ was heated in a small sealed tube at $100{ }^{\circ} \mathrm{C}$ for $72 \mathrm{~h}$. The mixture was cooled to rt, dry-loaded onto a small amount of silica, and then purified by column chromatography ( $3 \% \mathrm{Et}_{2} \mathrm{O}$ in petrol) to give arylcyclopropane $27(153 \mathrm{mg}, 0.48 \mathrm{mmol}, 48 \%)$ as a colorless oil, $R_{\mathrm{f}}=0.4\left(3 \% \mathrm{Et}_{2} \mathrm{O}\right.$ in petrol), and recovered starting material 23 (221 mg, 0.44 mmol, 44\%); IR (film) 3034w, 2954s (C-H), 2857s (C- 
O), 1614m (Ar), 1516s, 1464s, 1442m, 1361m, 1301m, 1255s, 1169s, 1078s, 1035s; ${ }^{1} \mathrm{H}$ NMR (400 MHz) $0.11\left(\mathrm{~s}, 3 \mathrm{H}, \mathrm{SiCH}_{3}\right), 0.12\left(\mathrm{~s}, 3 \mathrm{H}, \mathrm{SiCH}_{3}\right), 0.94$ (s, 9H, $\left.\mathrm{Si}\left(\mathrm{CH}_{3}\right)_{3}\right), 1.48(\mathrm{t}, 1 \mathrm{H}, \mathrm{H}-6, J=4.0)$, 1.50 - 1.53 (m, 1H, H-3), 1.57 - 1.63 (m, 2H, H-1, H-3'), 1.69 - 1.72 (m, 1H, H-5), 1.82 (dd, 1H, H4, $J=7.6,12.4), 2.06-2.15\left(\mathrm{~m}, 1 \mathrm{H}, \mathrm{H}-4^{\prime}\right), 3.79\left(\mathrm{~s}, 3 \mathrm{H}, \mathrm{OCH}_{3}\right), 4.39$ (d, 1H, H-2, J = 4.8), $6.82(\mathrm{~d}$, $2 \mathrm{H}, 2 \times \mathrm{Ar}-\mathrm{H}, J=9.2), 6.97(\mathrm{~d}, 2 \mathrm{H}, 2 \times \mathrm{Ar}-\mathrm{H}, J=8.8) ;{ }^{13} \mathrm{C} \mathrm{NMR}(100 \mathrm{MHz})-4.5\left(\mathrm{SiCH}_{3}\right), 18.3$ $\left(\mathrm{SiC}\left(\mathrm{CH}_{3}\right)_{3}\right), 23.5$ (C-6), $25.6\left(\mathrm{SiC}\left(\mathrm{CH}_{3}\right)_{3}\right), 26.0$ (C-4), 28.7 (C-5), $32.1(\mathrm{C}-3), 36.3(\mathrm{C}-1), 55.3$ $\left(\mathrm{OCH}_{3}\right), 75.0$ (C-2), 113.7 (Ar-C), 126.4 (Ar-C), 134.7 (Ar-C), 157.5 (Ar-C); MS (EI+) m/z: 318.3 $\left(\mathrm{M}^{+}, 1 \%\right), 261.2(9 \%), 187.2(11 \%), 160.1(70 \%), 145.1(9 \%), 129.1(15 \%), 121.1(46 \%)$; HRMS $m / z: \mathrm{M}^{+}$found 318.2011, $\mathrm{C}_{19} \mathrm{H}_{30} \mathrm{O}_{2} \mathrm{Si}$ requires 318.2010.

\section{Model study of (+)- $\beta$-cuparenone}

\section{Formation of 5-phenylbicyclo[3.1.0]hexan-2-ol 5c using substoichiometric TMP}

n-BuLi (1.6 $\mathrm{M}$ in hexanes, $1.07 \mathrm{~mL}, 1.72 \mathrm{mmol}, 2.0$ equiv.) was added slowly over $40 \mathrm{~min}$ to a stirred solution of epoxide $4 \mathbf{c}(150 \mathrm{mg}, 0.86 \mathrm{mmol})$ and tetramethylpiperidine $(0.073 \mathrm{ml}, 0.43 \mathrm{mmol}$, 0.5 equiv.) in $t$-BuOMe $(4 \mathrm{ml})$ at $0--5{ }^{\circ} \mathrm{C}$. The resulting pale-yellow mixture was stirred at the same temperature for $4.5 \mathrm{~h}$. The reaction was then quenched with aq. $\mathrm{HCl}$ (3 M, 2.0 equiv.), diluted with $\mathrm{Et}_{2} \mathrm{O}(10 \mathrm{~mL})$ and washed twice with aq. $\mathrm{HCl}(3 \mathrm{M}, 0.5$ equiv.). The aqueous layer was back extracted with $\mathrm{Et}_{2} \mathrm{O}(2 \times 5 \mathrm{~mL})$, and the combined organic layers were dried $\left(\mathrm{MgSO}_{4}\right)$ and concentrated in vacuo. Purification of the residue by column chromatography $\left(30 \% \mathrm{Et}_{2} \mathrm{O}\right.$ in petrol) gave 5c (120 mg, $0.69 \mathrm{mmol}, 80 \%)$ as a colorless oil. Data as above. [When the same reaction was performed under identical conditions using 1.1 equiv. of $n$-BuLi added slowly over 20 min, it gave 5c $(79 \mathrm{mg}, 53 \%, 58 \%$ brsm $)]$. 


\section{Asymmetric synthesis of $(+)-\beta$-cuparenone $(+)-28$}

$(+)$ - $\beta$-Cuparenone $(+)-28$

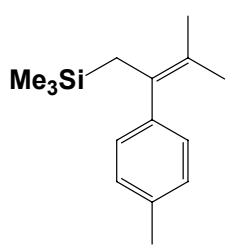

31

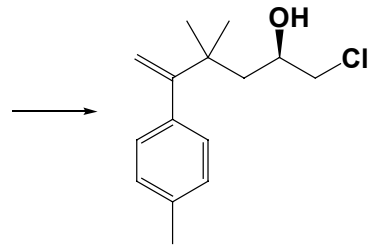

$(R)-33$

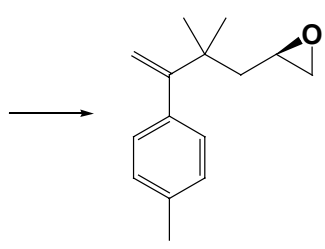

$(R)-30$
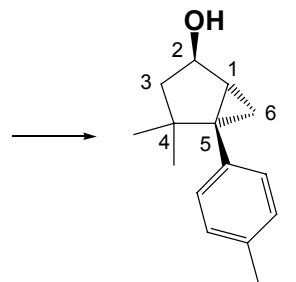

$(-)-34$

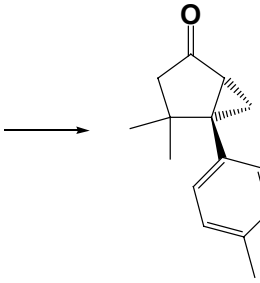

$(+)-29$

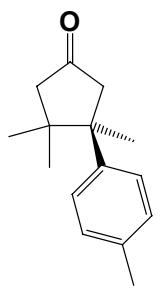

$(+)-28$

2-p-Tolyl-3-methyl-but-2-en-1-yl-trimethylsilane 31

A $50 \mathrm{~mL}$ round-bottom flask containing $\mathrm{Pd}(\mathrm{dba})_{2}(518 \mathrm{mg}, 0.9 \mathrm{mmol})$ was purged with Argon $(\times 3)$. To the flask were then added toluene $(54 \mathrm{~mL}), 4$-iodotoluene $(3.92 \mathrm{~g}, 18 \mathrm{mmol}), 3$-methyl-1,2butadiene $(3.57 \mathrm{~mL}, 36.0 \mathrm{mmol})$, and tributyl(trimethylsilyl)stannane $(6.28 \mathrm{~mL}, 18 \mathrm{mmol})$. The reaction mixture was heated with stirring at $80{ }^{\circ} \mathrm{C}$ for $7 \mathrm{~h}$. The solution changed rapidly from purple red to pale yellow in the first few minutes and maintained the same color during the reaction. As the reaction approached completion, a black precipitate of palladium metal surrounding the wall of the flask appeared gradually. The solution was filtered through celite, the filtrate was concentrated, and the residue was purified by distillation $\left(90{ }^{\circ} \mathrm{C}, 0.045 \mathrm{mmHg}\right)$ to give allylsilane $31(2.08 \mathrm{~g}, 8.95$ mmol, $50 \%)$ as a colorless oil, $R_{\mathrm{f}}=0.88\left(20 \% \mathrm{Et}_{2} \mathrm{O}\right.$ in petrol); IR (film) $2922 \mathrm{~s}, 1510 \mathrm{~m}, 1248 \mathrm{~s}$, 1166w, 833s; ${ }^{1} \mathrm{H}$ NMR (200 MHz) -0.15 (s, 9H, Si( $\left.\left(\mathrm{CH}_{3}\right)_{3}\right), 1.62$ (s, 3H, $\left.\mathrm{CH}_{3}\right), 1,76\left(\mathrm{~s}, 3 \mathrm{H}, \mathrm{CH}_{3}\right)$, $1.89\left(\mathrm{~s}, 2 \mathrm{H}, \mathrm{CH}_{2} \mathrm{Si}\right), 2.34\left(\mathrm{~s}, 3 \mathrm{H}, \mathrm{CH}_{3} \mathrm{Ph}\right), 7.03(\mathrm{~d}, 2 \mathrm{H}, 2 \times \mathrm{Ar}-\mathrm{H}, J=8.2), 7.09(\mathrm{~d}, 2 \mathrm{H}, 2 \times \mathrm{Ar}-\mathrm{H}, J=$ 8.2); ${ }^{13} \mathrm{C}$ NMR $(100 \mathrm{MHz})-0.9\left(\mathrm{Si}\left(\mathrm{CH}_{3}\right)_{3}\right), 21.1\left(\left(\mathrm{CH}_{3}\right)_{2} \mathrm{C}=\mathrm{C}\right), 22.1\left(\mathrm{CH}_{3} \mathrm{Ph}\right), 25.7\left(\mathrm{CH}_{2}\right), 123.5$ $\left(\left(\mathrm{CH}_{3}\right)_{2} \mathrm{C}=\mathrm{C}\right), 128.3(2 \times \mathrm{Ar}-\mathrm{C}), 129.0(2 \times \mathrm{Ar}-\mathrm{C}), 132.2(\mathrm{Ar}-\mathrm{C}), 135.1\left(\left(\mathrm{CH}_{3}\right)_{2} \mathrm{C}=\mathrm{C}\right), 142.1(\mathrm{Ar}-\mathrm{C})$; MS (CI+) m/z: $233\left(\mathrm{MH}^{+}, 100 \%\right), 232$ (27\%), 90 (84\%), 73 (27\%); HRMS m/z: $\mathrm{MH}^{+}$found 233.1716, $\mathrm{C}_{15} \mathrm{H}_{25} \mathrm{Si}$ requires 233.1726. 
(R)-1-Chloro-4,4-dimethyl-5-p-tolyl-hex-5-en-2-ol (R)-33

To a stirred solution of allylsilane $\mathbf{3 1}(284 \mathrm{mg}, 1.22 \mathrm{mmol})$ and $(R)$-epichlorohydrin $(\boldsymbol{R})-\mathbf{3 2}(0.12$ $\mathrm{mL}, 140 \mathrm{mg}, 1.46 \mathrm{mmol})$ in $\mathrm{CH}_{2} \mathrm{Cl}_{2}(2.6 \mathrm{~mL})$ at $-78{ }^{\circ} \mathrm{C}$ was added a solution of $\mathrm{TiCl}_{4}(1 \mathrm{M}$ in $\left.\mathrm{CH}_{2} \mathrm{Cl}_{2}, 1.46 \mathrm{~mL}, 1.46 \mathrm{mmol}\right)$ through the cold inner surface of the flask over a period of $2-3 \mathrm{~min}$. The violet-red mixture was stirred at $-78{ }^{\circ} \mathrm{C}$ for $30 \mathrm{~min}$ and then removed from the cold bath. $30 \%$ aq. $\mathrm{NaOH}(0.8 \mathrm{~mL})$ and $\mathrm{Et}_{2} \mathrm{O}(3.73 \mathrm{~mL})$ were immediately added, and the resulting mixture was stirred vigorously while being allowed to warm to rt. The organic phase was separated, washed with half-saturated aq. $\mathrm{NaCl}(2 \times 10 \mathrm{~mL})$, dried $\left(\mathrm{MgSO}_{4}\right)$, filtered and evaporated in vacuo. Purification of the residue by column chromatography $\left(20 \% \mathrm{Et}_{2} \mathrm{O}\right.$ in petrol) gave chlorohydrin $(\boldsymbol{R})-33$ (184 mg, $0.73 \mathrm{mmol}, 60 \%)$ as a colorless oil, $R_{\mathrm{f}}=0.58\left(20 \% \mathrm{Et}_{2} \mathrm{O}\right.$ in petrol $) ;[\alpha]_{\mathrm{D}}^{21}=+0.48\left(c, 1.0\right.$ in $\left.\mathrm{CHCl}_{3}\right)$; IR (film) 3442m, 2966s, 1623w, 1511m, 1430m, 1382m, 1090m, 909s, 825s; ${ }^{1} \mathrm{H}$ NMR (400 MHz) $1.20\left(\mathrm{~s}, 3 \mathrm{H}, \mathrm{CH}_{3}\right), 1.23\left(\mathrm{~s}, 3 \mathrm{H}, \mathrm{CH}_{3}\right), 1.55\left(\mathrm{dd}, 1 \mathrm{H}, \mathrm{CH}_{\mathrm{A}} H_{B} \mathrm{CHOH}, J=2.5,14.6\right), 1.71(\mathrm{dd}, 1 \mathrm{H}$, $\left.\mathrm{CH}_{A} \mathrm{H}_{\mathrm{B}} \mathrm{CHOH}, J=8.1,14.6\right), 2.34($ br s, $\mathrm{OH}), 2.36\left(\mathrm{~s}, 3 \mathrm{H}, \mathrm{CH}_{3} \mathrm{Ph}\right), 3.44\left(\mathrm{dd}, 1 \mathrm{H}, \mathrm{CH}_{\mathrm{A}} H_{B} \mathrm{Cl}, J=7.3\right.$, 11.0), $3.53\left(\mathrm{dd}, 1 \mathrm{H}, \mathrm{CH}_{A} \mathrm{H}_{\mathrm{B}} \mathrm{Cl}, J=3.5,11.0\right), 3.99-4.03(\mathrm{~m}, 1 \mathrm{H}, \mathrm{CHOH}), 4.96\left(\mathrm{~d}, 1 \mathrm{H}, \mathrm{H}_{\mathrm{A}} H_{B} \mathrm{C}=\mathrm{C}, J\right.$ $=1.5), 5.27\left(\mathrm{~d}, 1 \mathrm{H}, H_{A} \mathrm{H}_{\mathrm{B}} \mathrm{C}=\mathrm{C}, J=1.5\right), 7.09(\mathrm{~d}, 2 \mathrm{H}, J=8.3,2 \times \mathrm{Ar}-\mathrm{H}), 7.12(\mathrm{~d}, 2 \mathrm{H}, J=8.3,2 \times \operatorname{Ar}-$ $\mathrm{H}) ;{ }^{13} \mathrm{C}$ NMR $(100 \mathrm{MHz}) 21.0\left(\mathrm{CH}_{3} \mathrm{Ph}\right), 28.3\left(\left(\mathrm{CH}_{3}\right)_{2} \mathrm{C}\right), 38.7\left(\left(\mathrm{CH}_{3}\right)_{2} \mathrm{C}\right), 44.8\left(\mathrm{CH}_{2} \mathrm{CHOH}\right), 51.4$ $\left(\mathrm{CH}_{2} \mathrm{Cl}\right), 69.5(\mathrm{CHOH}), 114.3\left(\mathrm{H}_{2} \mathrm{C}=\mathrm{C}\right), 128.2(2 \times \mathrm{Ar}-\mathrm{C}), 128.8(2 \times \mathrm{Ar}-\mathrm{C}), 136.2(\mathrm{Ar}-\mathrm{C}), 139.8$ (Ar-C), $158.1\left(\mathrm{H}_{2} \mathrm{C}=C\right)$; MS (CI+) m/z: $270\left(\mathrm{MNH}_{4}^{+}, 100 \%,\right), 253\left(\mathrm{MH}^{+}, 27 \%,\right), 235(12 \%), 199$ (64\%), 160 (7\%), 135 (26\%), 119 (15\%); HRMS m/z: $\mathrm{MNH}_{4}^{+}$found 270.1628, $\mathrm{C}_{15} \mathrm{H}_{25} \mathrm{NOCl}$ requires 270.1625 .

\section{(R)-1,2-Epoxy-4,4-dimethyl-5-p-tolyl-5-hexene (R)-30}

Powdered $\mathrm{NaOH}(29 \mathrm{mg}, 0.72 \mathrm{mmol})$ was added to a stirred solution of chlorohydrin $(\boldsymbol{R})-33$ (150 $\mathrm{mg}, 0.59 \mathrm{mmol})$ in $\mathrm{MeOH}(0.56 \mathrm{~mL})$ at $0{ }^{\circ} \mathrm{C}$. The resulting mixture was stirred at $\mathrm{rt}$ for $1 \mathrm{~h}$, diluted with $\mathrm{Et}_{2} \mathrm{O}(30 \mathrm{~mL})$, and washed with water $(30 \mathrm{~mL})$. The organic phase was dried $\left(\mathrm{MgSO}_{4}\right)$ and concentrated in vacuo. The residue was purified by flash column chromatography $\left(20 \% \mathrm{Et}_{2} \mathrm{O}\right.$ in petrol) to give epoxide $(\boldsymbol{R})-\mathbf{3 0}(128 \mathrm{mg}, 0.59 \mathrm{mmol}, 99 \%)$ as colorless oil, $R_{\mathrm{f}}=0.71\left(20 \% \mathrm{Et}_{2} \mathrm{O}\right.$ in petrol); $[\alpha]_{\mathrm{D}}{ }^{21}=+3.4\left(c, 1.0\right.$ in $\left.\mathrm{CHCl}_{3}\right)$; IR (film) $2968 \mathrm{~m}, 1592 \mathrm{~m}, 824 \mathrm{~s} ;{ }^{1} \mathrm{H} \mathrm{NMR}(400 \mathrm{MHz}) 1.21(\mathrm{~s}$, 
$\left.3 \mathrm{H}, \mathrm{CH}_{3}\right), 1.23\left(\mathrm{~s}, 3 \mathrm{H}, \mathrm{CH}_{3}\right), 1.61-1.63\left(\mathrm{~m}, 2 \mathrm{H}, \mathrm{CH}_{2} \mathrm{CHO}\right), 2.36\left(\mathrm{~s}, 3 \mathrm{H}, \mathrm{CH}_{3} \mathrm{Ph}\right), 2.43$ (dd, 1H, $\left.\mathrm{CH}_{\mathrm{A}} H_{B} \mathrm{O}, J=2.8,5.0\right), 2.77\left(\mathrm{dd}, 1 \mathrm{H}, \mathrm{CH}_{A} \mathrm{H}_{\mathrm{B}} \mathrm{O}, J=4.0,5.0\right), 3.00-3.04(\mathrm{~m}, 1 \mathrm{H}, \mathrm{CHO}), 4.92(\mathrm{~d}$, $\left.1 \mathrm{H}, H_{A} \mathrm{H}_{\mathrm{B}} \mathrm{C}=\mathrm{C}, J=1.5\right), 5.24\left(\mathrm{~d}, 1 \mathrm{H}, \mathrm{H}_{\mathrm{A}} H_{B} \mathrm{C}=\mathrm{C}, J=1.5\right), 7.06(\mathrm{~d}, 2 \mathrm{H}, 2 \times \mathrm{Ar}-\mathrm{H}, J=8.0), 7.12(\mathrm{~d}$, $2 \mathrm{H}, 2 \times \mathrm{Ar}-\mathrm{H}, J=8.0) ;{ }^{13} \mathrm{C} \mathrm{NMR}(100 \mathrm{MHz}) 21.0\left(\mathrm{CH}_{3} \mathrm{Ph}\right), 27.7\left(\mathrm{CH}_{3} \mathrm{CCH}_{3}\right), 27.9\left(\mathrm{CH}_{3} \mathrm{CCH}_{3}\right)$, $38.9\left(\mathrm{C}\left(\mathrm{CH}_{3}\right)_{2}\right), 43.5\left(\mathrm{CH}_{2} \mathrm{CHO}\right), 46.9\left(\mathrm{CH}_{2} \mathrm{O}\right), 49.7(\mathrm{CHO}), 113.7\left(\mathrm{CH}_{2}=\mathrm{C}\right), 128.2$ (Ar-C), 128.9 (Ar-C), 136.1, $139.9\left(2 \times\right.$ Ar-C), $157.4\left(\mathrm{H}_{2} \mathrm{C}=C\right)$; $\mathrm{MS}(\mathrm{CI}+) \mathrm{m} / z: 234\left(\mathrm{MNH}_{4}^{+}, 13 \%\right), 217\left(\mathrm{MH}^{+}\right.$, $100 \%$,), 199 (17\%), $160(14 \%)$; HRMS m/z: $\mathrm{MH}^{+}$found 217.1599, $\mathrm{C}_{15} \mathrm{H}_{21} \mathrm{O}$ requires 217.1592.

Formation of $(1 R, 2 R, 5 R)-4,4$-dimethyl-5-p-tolyl-bicyclo[3.1.0] hexan-2-ol (-)-34 from epoxide (R)30 under standard conditions

Following the typical cyclopropanation procedure, epoxide $(\boldsymbol{R})-\mathbf{3 0}(100 \mathrm{mg}, 0.46 \mathrm{mmol})$ was treated with LTMP in $t$-BuOMe for $20 \mathrm{~h}$ to give bicyclic alcohol (-)-34 (71 $\mathrm{mg}, 0.33 \mathrm{mmol}, 72 \%)$ as a colorless oil, $R_{\mathrm{f}}=0.3\left(30 \% \mathrm{Et}_{2} \mathrm{O}\right.$ in petrol); $[\alpha]_{\mathrm{D}}{ }^{21}=-3\left(c, 1.0\right.$ in $\left.\mathrm{CHCl}_{3}\right)$; IR (film) $3356 \mathrm{~m}, 2956 \mathrm{~s}$, 1516m, 1465m, 1034m, 998m, 820m; ${ }^{1} \mathrm{H}$ NMR (400 MHz) 0.60 - 0.62 (m, 1H, H-6), 0.66 (dd, 1H, H-6', $J=4.9,8.5), 1.00\left(\mathrm{~s}, 3 \mathrm{H}, \mathrm{CH}_{3}\right), 1.07$ (s, 3H, $\left.\mathrm{CH}_{3}\right), 1.56-1.57$ (m, 2H, H-3, H-3'), 1.68 (s, 1H, $\mathrm{OH}), 1.73(\mathrm{dd}, 1 \mathrm{H}, \mathrm{CH}-1, J=3.8,8.6), 2.35\left(\mathrm{~s}, 3 \mathrm{H}, \mathrm{CH}_{3} \mathrm{Ph}\right), 4.32-4.33(\mathrm{~m}, 1 \mathrm{H}, \mathrm{H}-2), 7.11(\mathrm{~d}, 2 \mathrm{H}, 2$ $\times \operatorname{Ar}-\mathrm{H}, J=7.8), 7.26(\mathrm{~d}, 2 \mathrm{H}, 2 \times \mathrm{Ar}-\mathrm{H}, J=7.8) ;{ }^{13} \mathrm{C}$ NMR $(100 \mathrm{MHz}) 13.9(\mathrm{C}-6), 21.1\left(\mathrm{CH}_{3} \mathrm{Ph}\right)$, $\left.25.6\left(\mathrm{CH}_{3}\right), 29.1\left(\mathrm{CH}_{3}\right), 32.0(\mathrm{C}-1), 41.2(\mathrm{C}-4), 42.3(\mathrm{C}-5), \mathrm{CPh}\right), 47.3(\mathrm{C}-3), 74.5(\mathrm{C}-2), 128.4(\mathrm{Ar}-$ C), 130.8 (Ar-C), 135.9, $138.2\left(2 \times\right.$ Ar-C); $\mathrm{MS}(\mathrm{CI}+) \mathrm{m} / \mathrm{z}: 199\left(\left[\mathrm{M}-\mathrm{H}_{2} \mathrm{O}\right] \mathrm{H}^{+}, 100 \%\right), 198(52 \%)$, $183(15 \%)$; HRMS $m / z: \mathrm{M}^{+}$found 216.1513, $\mathrm{C}_{15} \mathrm{H}_{20} \mathrm{O}$ requires 216.1514.

Formation of $(1 R, 2 R, 5 R)-4,4-d i m e t h y l-5$-p-tolyl-bicyclo[3.1.0]hexan-2-ol (-)-34 from chlorohydrin (R)-33

Following the typical cyclopropanation procedure, chlorohydrin $(\boldsymbol{R})-33$ (253 $\mathrm{mg}, 1.00 \mathrm{mmol})$ was treated with LTMP (3.5 equiv.) in $t$-BuOMe for $20 \mathrm{~h}$ to give bicyclic alcohol (-)-34 (128 $\mathrm{mg}, 0.59$ mmol, 59\%) as a colorless oil. Data as above. 
Formation of $(1 R, 2 R, 5 R)-4,4$-dimethyl-5-p-tolyl-bicyclo[3.1.0] hexan-2-ol (-)-34 from epoxide (R)30 using substoichiometric TMP

$n$-BuLi (1.6 M in hexanes, $0.58 \mathrm{~mL}, 0.93 \mathrm{mmol}$ ) was added very slowly over $25 \mathrm{~min}$ to a stirred solution of epoxide $(\boldsymbol{R})-30(100 \mathrm{mg}, 0.46 \mathrm{mmol})$ and tetramethylpiperidine (33 $\mathrm{mg}, 0.23 \mathrm{mmol}$, $0.039 \mathrm{~mL})$ in $t$-BuOMe $(2.3 \mathrm{~mL})$ at $-2{ }^{\circ} \mathrm{C}$. The resulting pale-yellow mixture was stirred at the same temperature for $4.5 \mathrm{~h}$ (complete consumption of the epoxide). The reaction was then quenched with aq. $\mathrm{HCl}\left(3 \mathrm{M}, 2\right.$ equiv.), diluted with $\mathrm{Et}_{2} \mathrm{O}(10 \mathrm{~mL})$ and washed twice with aq. $\mathrm{HCl}(3 \mathrm{M}, 0.5$ equiv.); the aqueous phase was extracted twice with $\mathrm{Et}_{2} \mathrm{O}(2 \times 5 \mathrm{~mL})$ and the combined organic phases dried $\left(\mathrm{MgSO}_{4}\right)$ and concentrated in vacuo. Purification of the residue by column chromatography (30\% $\mathrm{Et}_{2} \mathrm{O}$ in petrol) gave (-)-34 (84 mg, $\left.0.39 \mathrm{mmol}, 85 \%\right)$ as a colorless oil. Data as above. [When the same reaction was performed under identical conditions using 1.1 equiv. of $n$ BuLi added slowly over 13 min, it gave (-)-34 in $72 \%$ yield].

\section{(1R,5R)-4,4-dimethyl-5-p-tolyl-bicyclo[3.1.0]hexan-2-one (+)-29 ${ }^{[16]}$}

TPAP $(5.3 \mathrm{mg}, 0.015 \mathrm{mmol})$ was added to a stirred solution of bicyclic alcohol (-)-34 (65 mg, 0.30 mmol), 4-methylmorpholine $N$-oxide $(71 \mathrm{mg}, 0.60 \mathrm{mmol})$, and powdered $3 \AA$ molecular sieves $(83$ $\mathrm{mg})$ in $\mathrm{CH}_{2} \mathrm{Cl}_{2}(2.2 \mathrm{~mL})$ at $20{ }^{\circ} \mathrm{C}$. After $2 \mathrm{~h}$ the mixture was filtered through a short pad of silica $(2.5$ $\times 8 \mathrm{~cm})$ and eluted with $\mathrm{Et}_{2} \mathrm{O}(100 \mathrm{ml})$. The solvent was evaporated in vacuo to give ketone (+)-29 $(64 \mathrm{mg}, 0.30 \mathrm{mmol}, 99 \%)$ as a white solid, $R_{\mathrm{f}}=0.59\left(30 \% \mathrm{Et}_{2} \mathrm{O}\right.$ in petrol); $[\alpha]_{\mathrm{D}}{ }^{20}=+19.5(c, 1.0$ in $\left.\mathrm{CHCl}_{3}\right),\left\{\right.$ lit. $^{[16]}[\alpha]_{\mathrm{D}}=-16.1,\left(c, 2.6\right.$ in $\mathrm{CHCl}_{3}$ for $(-)$-enantiomer $\left.)\right\}$; IR $(\mathrm{KBr}) 1717 \mathrm{~s} ;{ }^{1} \mathrm{H}$ NMR $(400$ MHz) $0.92\left(\mathrm{~s}, 3 \mathrm{H}, \mathrm{CH}_{3}\right), 1.23\left(\mathrm{~s}, 3 \mathrm{H}, \mathrm{CH}_{3}\right), 1.36-1.40\left(\mathrm{~m}, 1 \mathrm{H}, \mathrm{CH}_{\mathrm{A}} H_{B} \mathrm{CHCO}\right), 1.58(\mathrm{dd}, 1 \mathrm{H}$, $\left.\mathrm{CH}_{A} \mathrm{H}_{\mathrm{B}} \mathrm{CHCO}, J=3.0,4.5\right), 1.90\left(\mathrm{~d}, 1 \mathrm{H}, \mathrm{CH}_{\mathrm{A}} H_{B} \mathrm{CO}, J=17.3\right), 2.12(\mathrm{dd}, 1 \mathrm{H}, \mathrm{CHCO}, J=8.6,2.8)$, $2.21\left(\mathrm{~d}, 1 \mathrm{H}, \mathrm{CH}_{A} \mathrm{H}_{\mathrm{B}} \mathrm{CO}, J=17.3\right), 2.35\left(\mathrm{~s}, 3 \mathrm{H}, \mathrm{CH}_{3} \mathrm{Ph}\right), 7.12(\mathrm{~d}, 2 \mathrm{H}, J=8.2), 7.20(\mathrm{~d}, 2 \mathrm{H}, J=8.2)$; ${ }^{13} \mathrm{C}$ NMR (100 MHz) $20.7\left(\mathrm{CH}_{2} \mathrm{CHCO}\right), 21.1\left(\mathrm{CH}_{3} \mathrm{Ph}\right), 24.1\left(\mathrm{CH}_{3}\right), 28.4\left(\mathrm{CH}_{3}\right), 35.2(\mathrm{CHCO}), 38.9$, $47.1\left(\mathrm{C}\left(\mathrm{CH}_{3}\right)_{2}, \mathrm{CPh}\right), 48.1\left(\mathrm{CH}_{2} \mathrm{CO}\right), 128.7(2 \times \mathrm{Ar}-\mathrm{C}), 130.4(2 \times \mathrm{Ar}-\mathrm{C}), 135.1,137.2(2 \times \mathrm{Ar}-$ $\left.\mathrm{C}_{\text {quat }}\right), 213.2(\mathrm{CO})$; MS (CI+) m/z: $232\left(\mathrm{MNH}_{4}^{+}, 87 \%\right), 215\left(\mathrm{MH}^{+}, 100 \%\right), 158(17 \%)$; HRMS m/z: $\mathrm{MNH}_{4}{ }^{+}$found 232.1693, $\mathrm{C}_{15} \mathrm{H}_{22} \mathrm{NO}$ requires 232.1701. 
(R)-(+)- $\beta$-cuparenone (+)-28

Small pieces of lithium wire (20 mg, $2.88 \mathrm{mmol}$, prewashed with anhydrous pentane) were placed in a three-necked flask under argon. Ammonia $(\sim 8 \mathrm{~mL})$ was condensed into the flask at $-78{ }^{\circ} \mathrm{C}$ and a solution of ketone $(+)-29(65 \mathrm{mg}, 0.30 \mathrm{mmol})$ and $t-\mathrm{BuOH}(0.18 \mathrm{~mL})$ in anhydrous $\mathrm{Et}_{2} \mathrm{O}(3.6 \mathrm{~mL})$ were added. After $15 \mathrm{~min}, \mathrm{NH}_{4} \mathrm{Cl}(600 \mathrm{mg})$ was added to the blue solution. Ammonia was allowed to evaporate by warming to $\mathrm{rt}$ and the remaining solution was diluted with $\mathrm{Et}_{2} \mathrm{O}(30 \mathrm{~mL})$. The solution was extracted with water $(30 \mathrm{~mL})$, the layers were separated and the aqueous layer was extracted with $\mathrm{Et}_{2} \mathrm{O}(3 \times 10 \mathrm{~mL})$. The combined organic layers were dried $\left(\mathrm{MgSO}_{4}\right)$ and concentrated in vacuo. Purification of the residue by column chromatography $\left(30 \% \mathrm{Et}_{2} \mathrm{O}\right.$ in petrol) gave (+)-28 (56 mg $0.259 \mathrm{mmol}, 85 \%)$ as a colorless oil, $R_{\mathrm{f}}=0.67\left(30 \% \mathrm{Et}_{2} \mathrm{O}\right.$ in petrol); $[\alpha]_{\mathrm{D}}{ }^{21}=$ $+42\left(c, 1\right.$ in $\left.\mathrm{CHCl}_{3}\right)$; lit. ${ }^{[17]}[\alpha]_{\mathrm{D}}{ }^{29}=+45\left(c, 1.4\right.$ in $\left.\mathrm{CHCl}_{3}\right)$, lit. ${ }^{[18]}[\alpha]_{\mathrm{D}}{ }^{23}=+44.4\left(c, 2.47\right.$ in $\left.\mathrm{CHCl}_{3}\right)$; IR (film) 2961s, 1742s (C=O), 1517m, 1457m, 1406m, 1203m, 1020m, 818m; ${ }^{1} \mathrm{H}$ NMR (200 MHz) $0.74\left(\mathrm{~s}, 3 \mathrm{H}, \mathrm{CH}_{3}\right), 1.24\left(\mathrm{~s}, 3 \mathrm{H}, \mathrm{CH}_{3}\right), 1.43\left(\mathrm{~s}, 3 \mathrm{H}, \mathrm{CH}_{3} \mathrm{CPh}\right), 2.28-2.39(\mathrm{~m}, 3 \mathrm{H}), 2.34(\mathrm{~s}, 3 \mathrm{H}$, $\left.\mathrm{CH}_{3} \mathrm{Ph}\right), 3.14(\mathrm{~d}, 1 \mathrm{H}, J=18.2), 7.14(\mathrm{~d}, 2 \mathrm{H}, 2 \times \mathrm{Ar}-\mathrm{H}, J=8.2), 7.21(\mathrm{~d}, 2 \mathrm{H}, 2 \times \mathrm{Ar}-\mathrm{H}, J=8.2) ;{ }^{13} \mathrm{C}$

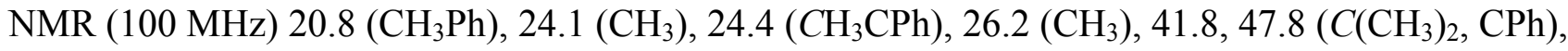
$50.7\left(\mathrm{CH}_{2}\right), 52.4\left(\mathrm{CH}_{2}\right), 126.5(2 \times \mathrm{Ar}-\mathrm{C}), 128.7(2 \times \mathrm{Ar}-\mathrm{C}), 135.8,141.2(2 \times \mathrm{Ar}-\mathrm{C}), 218.3(\mathrm{CO})$; MS (CI+) m/z: $234\left(\mathrm{MNH}_{4}^{+}, 100 \%,\right), 217\left(\mathrm{MH}^{+}, 13 \%\right), 132$ (97\%); HRMS m/z: $\mathrm{MNH}_{4}^{+}$found 234.1849, $\mathrm{C}_{15} \mathrm{H}_{24} \mathrm{NO}$ requires 234.1858.

Chiral GC (Cydex- $\beta$ column, $\left.100{ }^{\circ} \mathrm{C}, 1.0 \mathrm{mLmin}^{-1}\right)$ analysis of $(+)-28$ showed a resultant ee $=97 \%$

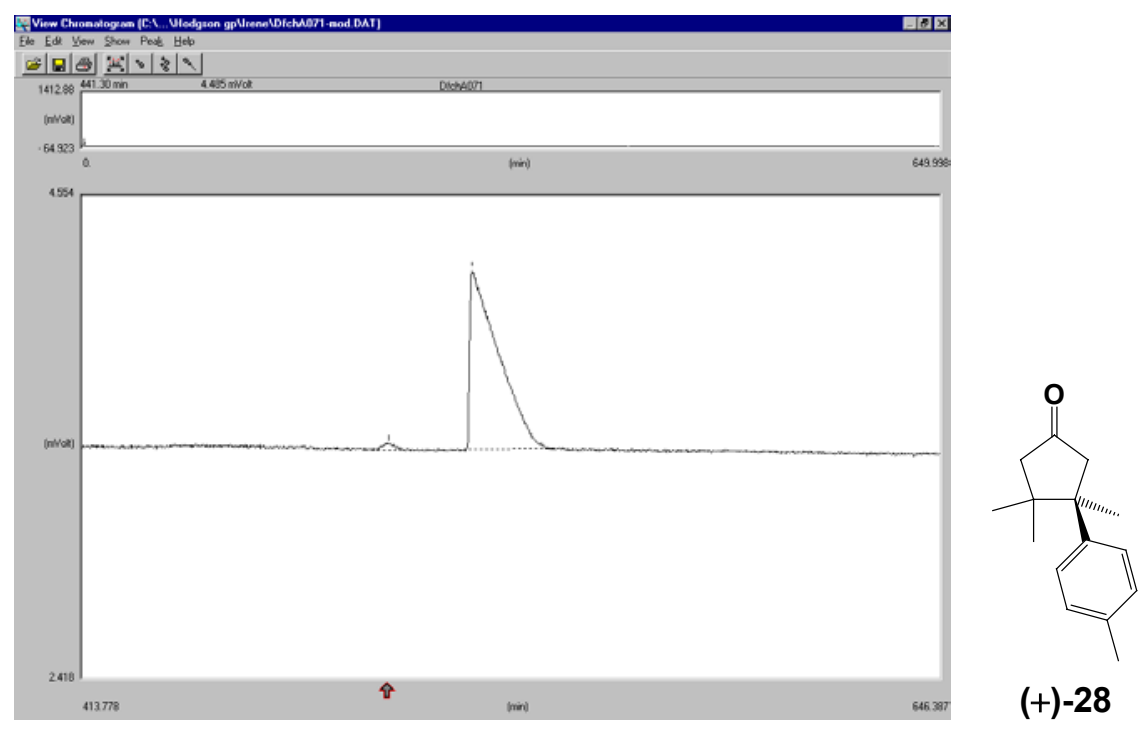


Peak Number \# Area \% Ret.Time Area BC

$\begin{array}{rrrr}1 & 1.5712 & 496.74 & 67504 \mathrm{mi} \\ 2 & 98.4288 & 519.49 & 4228792 \mathrm{mi} \\ \text { Totals } & 100.0000 & & 4296296\end{array}$

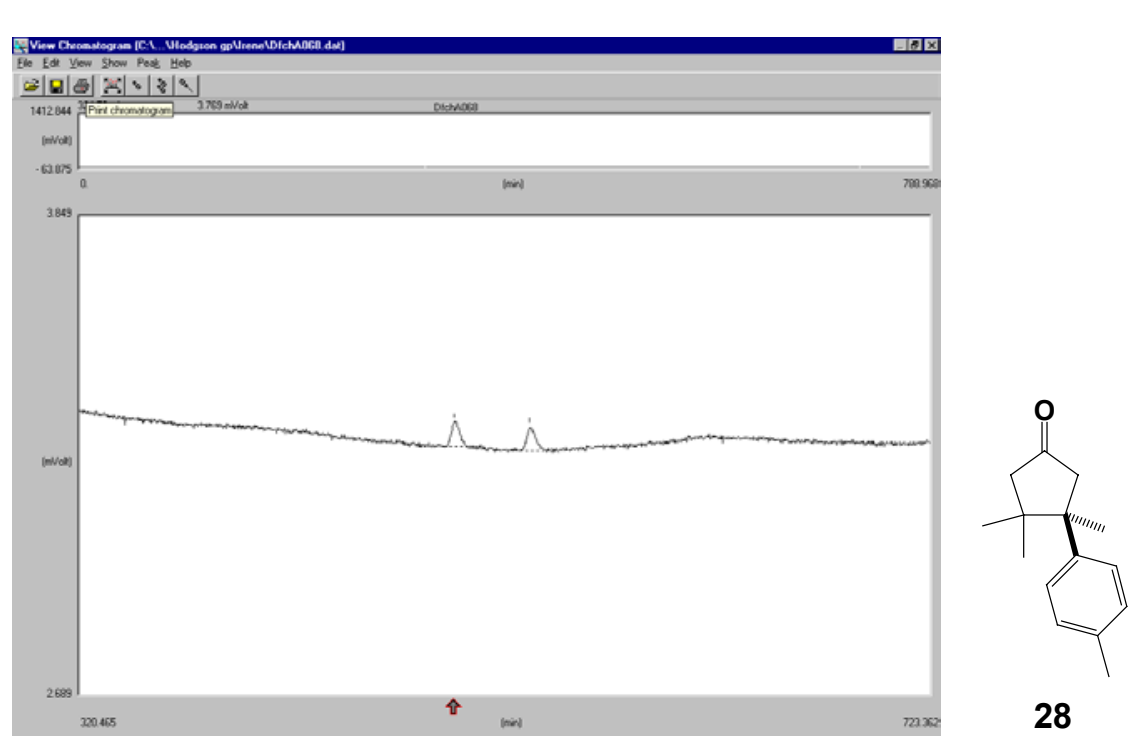

Peak Number \# Area \% Ret.Time Area BC

$\begin{array}{rrrl}1 & 49.9330 & 498.06 & 167686 \mathrm{mi} \\ 2 & 50.0670 & 533.54 & 168136 \mathrm{mi} \\ \text { Totals } & 100.0000 & & 335822\end{array}$

\section{5-Phenylbicyclo[3.1.0]hexan-2-one}

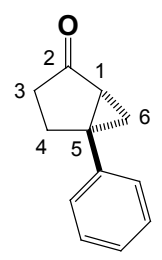

TPAP (24.3 mg, $0.069 \mathrm{mmol})$ was added to a stirred solution of alcohol $\mathbf{5 f}(241 \mathrm{mg}, 1.38$ mmol), 4-methylmorpholine $N$-oxide (325 mg, $2.77 \mathrm{mmol}$ ), and powdered $3 \AA$ molecular sieves (380 $\mathrm{mg})$ in $\mathrm{CH}_{2} \mathrm{Cl}_{2}(10.2 \mathrm{~mL})$ at $20{ }^{\circ} \mathrm{C}$. After $2 \mathrm{~h}$ the mixture was filtered through a short pad of silica $(10 \cdot 3 \mathrm{~cm})$ and eluted with $\mathrm{Et}_{2} \mathrm{O}(200 \mathrm{~mL})$; the organic solvent was evaporated in vacuo to give 5phenylbicyclo[3.1.0]hexan-2-one $(238 \mathrm{mg}, 100 \%)$ as a pale yellow oil, $R_{\mathrm{f}}=0.29\left(30 \% \mathrm{Et}_{2} \mathrm{O}\right.$ in petrol); IR (film) 2940w, 1728s (C=O), 1180m, 759m, 699m; ${ }^{1} \mathrm{H}$ NMR (400 MHz) 1.50 (dd, 1H, H$6 J=3.5,4.8), 1.61\left(\mathrm{ddt}, 1 \mathrm{H}, \mathrm{H}-6^{\prime}, J=1.4,4.8,9.3\right), 2.15(\mathrm{dd}, 1 \mathrm{H}, \mathrm{H}-1, J=3.5,9.3), 2.21$ - 2.31 (m, 
2H, 2·H-3), $2.3-2.49(\mathrm{~m}, 2 \mathrm{H}, 2 \times \mathrm{H}-4), 7.24-7.29(\mathrm{~m}, 3 \mathrm{H}, 3 \times \mathrm{Ar}-\mathrm{H}), 7.32-7.37(\mathrm{~m}, 2 \mathrm{H}, 2 \times \mathrm{Ar}-$ H); ${ }^{13} \mathrm{C}$ NMR (100 MHz) 21.1 (C-6), 29.0 (C-4), 33.4 (C-3), 35.7 (C-1), 37.1 (C-5), 126.9 (2 × ArC), $128.6(2 \times \mathrm{Ar}-\mathrm{C}), 141.7(\mathrm{Ar}-\mathrm{C}), 213.4(\mathrm{CO})$; $\mathrm{MS}(\mathrm{CI}+) \mathrm{m} / z: 190\left(20 \%, \mathrm{MNH}_{4}{ }^{+}\right), 173\left(\mathrm{MH}^{+}\right.$, 100\%), 130 (100\%), 115 (98\%), 91 (15\%); HRMS m/z: $\mathrm{MNH}_{4}{ }^{+}$found 190.1230, $\mathrm{C}_{12} \mathrm{H}_{16} \mathrm{NO}$ requires 190.1232.

\section{4-Phenyl cyclohexanone and 4-phenyl cyclohexanol}

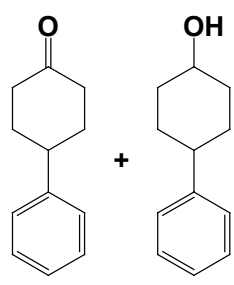

Small pieces of lithium wire $(82 \mathrm{mg}, 11.8 \mathrm{mmol}$, prewashed with anhydrous pentane) were placed in a three-necked flask. Ammonia $(\sim 30 \mathrm{~mL})$ was condensed into the flask at $78{ }^{\circ} \mathrm{C}$ and a solution of 5-phenylbicyclo[3.1.0]hexan-2-one (200 mg, $\left.1.16 \mathrm{mmol}\right)$ and $t$ - $\mathrm{BuOH}(0.70$ $\mathrm{mL})$ in $\mathrm{Et}_{2} \mathrm{O}(15 \mathrm{~mL})$ was added. The mixture was allowed to reflux for $15 \mathrm{~min}$ by removing the dry ice-acetone bath and then $\mathrm{NH}_{4} \mathrm{Cl}(1.47 \mathrm{~g})$ was added. The remaining ammonia was allowed to evaporate, the resulting solution was diluted with $\mathrm{Et}_{2} \mathrm{O}(50 \mathrm{~mL})$ and then extracted with water (50 $\mathrm{mL})$. The aqueous layer was extracted with $\mathrm{Et}_{2} \mathrm{O}(3 \times 10 \mathrm{~mL})$, the combined organic layers were dried $\left(\mathrm{MgSO}_{4}\right)$, filtered and evaporated in vacuo to give the crude product. Purification by column chromatography (30\% $\mathrm{Et}_{2} \mathrm{O}$ in petrol) gave 4-phenyl cyclohexanone ${ }^{[19]}(70 \mathrm{mg}, 0.40 \mathrm{mmol}, 35 \%)$ as a white solid, and 4-phenyl cyclohexanol ${ }^{[20]}(90 \mathrm{mg}, 0.51 \mathrm{mmol}, 44 \%$, as an inseparable mixture of two diastereoisomers, dr 6.7/3.3 determined by ${ }^{1} \mathrm{H}$ NMR analysis of the crude product) as a white solid.

Characterization data for 4-phenyl cyclohexanone:

$R_{\mathrm{f}}=0.47\left(30 \% \mathrm{Et}_{2} \mathrm{O}\right.$ in petrol); IR (KBr) 2960m, 1716s, 1450m, 1163m, 738s; ${ }^{1} \mathrm{H}$ NMR (400 MHz) $1.91-2.02\left(\mathrm{~m}, 2 \mathrm{H}, \mathrm{CH}_{2}\right), 2.21-2.26\left(\mathrm{~m}, 2 \mathrm{H}, \mathrm{CH}_{2}\right), 2.51-2.58\left(\mathrm{~m}, 4 \mathrm{H}, 2 \times \mathrm{CH}_{2} \mathrm{CO}\right), 3.00-3.08(\mathrm{~m}$, 1H, CH), 7.22 - $7.36(\mathrm{~m}, 5 \mathrm{H}, \mathrm{Ar}-\mathrm{H}) ;{ }^{13} \mathrm{C} \mathrm{NMR}(100 \mathrm{MHz}) 34.0\left(2 \times \mathrm{CH}_{2}\right), 41.4\left(2 \times \mathrm{CH}_{2} \mathrm{CO}\right), 42.8$ 
$(\mathrm{CH}), 126.6(\mathrm{Ar}-\mathrm{C}), 126.7(2 \times \mathrm{Ar}-\mathrm{C}), 128.6(2 \times \mathrm{Ar}-\mathrm{C}), 144.8(\mathrm{Ar}-\mathrm{C}), 211.2(\mathrm{C}=\mathrm{O}) ; \mathrm{MS}(\mathrm{CI}+)$ $m / z: 194\left(\mathrm{MNH}_{4}^{+}, 100 \%\right), 174$ (7\%); HRMS m/z: $\mathrm{MH}^{+}$found 175.1123, $\mathrm{C}_{12} \mathrm{H}_{15} \mathrm{O}$ requires 175.1123 .

Characterization data for 4-phenyl cyclohexanol:

$R_{\mathrm{f}}=0.19\left(30 \% \mathrm{Et}_{2} \mathrm{O}\right.$ in petrol); IR (KBr) 3375m, 2924s, 1494m, 1062m, 966w, 739m; ${ }^{1} \mathrm{H}$ NMR (400 MHz), inseparable mixture of two diastereoisomers: $1.27-1.33(\mathrm{~m}), 1.39-1.64(\mathrm{~m}), 1.73-$ $1.82(\mathrm{~m}), 1.92-2.13(\mathrm{~m}), 2.47-2.55(\mathrm{~m}), 2.60-2.72(\mathrm{~m}), 3.55-3.60(\mathrm{~m}, \mathrm{CHOH}), 3.67-3.74(\mathrm{~m}$, $\mathrm{CHOH}), 5.42$ - $5.44(\mathrm{~m}, \mathrm{OH}), 5.69$ - $5.77(\mathrm{~m}, \mathrm{OH}), 7.18$ - $7.22(\mathrm{~m}, \mathrm{Ar}-\mathrm{H})$, 7.27-7.32 (m, Ar-H); discernable data for major diastereoisomer: 3.67 - $3.74(\mathrm{~m}, \mathrm{CHOH}), 5.69-5.77$ (m, $\mathrm{OH})$; discernable data for minor diastereisomer: 3.55 - $3.60(\mathrm{~m}, \mathrm{CHOH}), 5.42-5.44(\mathrm{~m}, \mathrm{OH}) ;{ }^{13} \mathrm{C} \mathrm{NMR}$ $(100 \mathrm{MHz})$, inseparable mixture of two diastereisomer: $26.7\left(\mathrm{CH}_{2}\right), 27.4\left(\mathrm{CH}_{2}\right), 29.5\left(\mathrm{CH}_{2}\right), 32.4$ $\left(\mathrm{CH}_{2}\right), 35.7\left(\mathrm{CH}_{2}\right), 35.8\left(\mathrm{CH}_{2}\right), 43.4(\mathrm{CH}), 44.2(\mathrm{CH}), 70.6(\mathrm{CHOH}), 70.9(\mathrm{CHOH}), 117.0,124.3$, 124.4, 126.0, 126.7, 128.3 (Ar-C), 138.8 (Ar-C), 146.5 (Ar-C); discernable data for major diastereoisomer: $43.4(\mathrm{CH}), 70.6(\mathrm{CHOH}), 146.5($ Ar-C); discernable data for minor diastereisomer: $44.2(\mathrm{CH}), 70.9(\mathrm{CHOH}), 138.8(\mathrm{Ar}-\mathrm{C}) ; \mathrm{MS}(\mathrm{CI}+) \mathrm{m} / z: 159\left(\left[\mathrm{M}-\mathrm{H}_{2} \mathrm{O}\right] \mathrm{H}^{+}, 5 \%\right), 158\left(\mathrm{M}-\mathrm{H}_{2} \mathrm{O}\right.$, 100\%), 143 (8\%); HRMS m/z: $\mathrm{MNH}_{4}^{+}$found 194.1552, $\mathrm{C}_{12} \mathrm{H}_{20} \mathrm{NO}$ requires 194.1545.

\section{References}

[1] Hodgson, D. M.; Bray, C.; Kindon, N. D. J. Am. Chem. Soc. 2004, 126, 6870-6871.

[2] (a) Apparu, M.; Barrelle, M. Tetrahedron Lett. 1976, 17, 2837-2840. (b) Apparu, M.; Barrelle, M. Tetrahedron 1978, 34, 1691-1697.

[3] (a) Crandall, J. K.; Lin, L-H. C. J. Am. Chem. Soc. 1967, 89, 4526-4527. (b) Hodgson, D. M.; Chung, Y. K.; Paris, J-M. J. Am. Chem. Soc. 2004, 126, 8664-8665.

[4] Furrow, M. E.; Schaus, S. E.; Jacobsen, E. N.; J. Org. Chem. 1998, 63, 6776-6777. 
[5] Schaus, S. E.; Brandes, B. D.; Larrow, J. F.; Tokunaga, T.; Hansen, K. B.; Gould, A. E.;

Furrow, M. E.; Jacobsen, E. N. J. Am. Chem. Soc. 2002, 124, 1307-1315.

[6] Michnick, T. J.; Matteson, D. S. Synlett 1991, 631-632.

[7] Barbero, A.; Cuadrado, P.; Fleming, I.; González, A. M.; Pulido, F. J. J. Chem. Soc., Perkin Trans. 1 1992, 327-331.

[8] Kocienski, P.; Wadman, S.; Cooper, K. J. Am. Chem. Soc., 1989, 111, 2363-2365.

[9] Vares, L.; Koulov, A. V.; Smith, B. D. J. Org. Chem. 2003, 68, 10073-10078.

[10] Anand, N. K.; Carreira, E. M. J. Am. Chem. Soc. 2001, 123, 9687-9688.

[11] Crimmins, M. T.; Powell, M. T. J. Am. Chem. Soc. 2003, 125, 7592-7595.

[12] Reetz, M. T.; Steinbach, R.; Westermann, J.; Wenderoth, B. Chem. Ber. 1985, 118, 14411454.

[13] Boneva, S.; Vassilev, K. Chromatographia 1998, 47, 230-233.

[14] Vedejs, E.; Larsen, S. Org. Synth. Coll. Vol. VII, 1990, 277-280.

[15] Hayes, B. L. Microwave Synthesis Chemistry at the Speed of Light, CEM Publishing, 2002.

[16] Aavula, B. R.; Cui, Q.; Mash, E. Tetrahedron: Asymmetry 2000, 11, 4681-4686.

[17] Greene, A. E.; Charbonnier, F.; Luche, M. J.; Moyano, A. J. Am. Chem. Soc. 1987, 109, $4752-4753$.

[18] Asaoka, M.; Hayashibe, S.; Sonoda, S.; Takei, H. Tetrahedron Lett. 1990, 31, 4761-4764.

[19] Commercially available (Aldrich), CAS: 4894-75-1.

[20] Commercially available (Aldrich), CAS: 5437-46-7. 